\title{
Search for a Neutral Long-Lived Particle Decaying to $b$-Jets
}

\author{
Chad Johnson
}

Submitted in partial fulfillment of the

requirements for the degree of Doctor of Philosophy

in the Graduate School of Arts and Sciences

COLUMBIA UNIVERSITY

2009 
(C)2009

Chad Johnson

All Rights Reserved 


\section{ABSTRACT \\ Search for a Neutral Long-Lived Particle Decaying to $b$-Jets \\ Chad Johnson}

The existence of the Higgs boson is required by the Standard Model of particle physics, yet it has not been observed. The precise nature of the Higgs boson is unknown and the mechanism by which it interacts with known Standard Model particles is also not known. Long-lived, electrically neutral hadrons have recently been proposed in hidden-valley models and could constitute a pathway through which the Higgs boson communicates with the Standard Model. Such a scenario may provide a novel path to Higgs discovery at the Tevatron.

This thesis describes a search for a neutral, long-lived particle produced in decays of Higgs bosons in $p \bar{p}$ collisions at a center-of-mass energy of $\sqrt{s}=1.96 \mathrm{TeV}$, which decays to $b$-jets and lives long enough to travel at least $1.6 \mathrm{~cm}$ before decaying. This

analysis uses $3.65 \mathrm{fb}^{-1}$ of data recorded with the Run II DØ detector at the Fermilab Tevatron collider from April 2002 to August of 2008. We perform a search for eight possible hidden-valley scenarios resulting from a Higgs decay. No significant excess over background is observed and cross-section limits are placed at 95\% CL. 


\section{Contents}

1 Introduction 1

1.1 Historical Introduction . . . . . . . . . . . . . . . . . 1

1.2 Analysis Introduction . . . . . . . . . . . . . . . . . 3

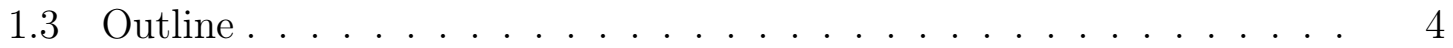

2 Theory and Experimental Introduction 5

2.1 Units . . . . . . . . . . . . . . . . . 5

2.2 The Standard Model . . . . . . . . . . . . . . . . . . . 6

2.2.1 Symmetries and Gauge Theories . . . . . . . . . . . . . . . 7

2.2.1.1 The QED Lagrangian . . . . . . . . . . . 7

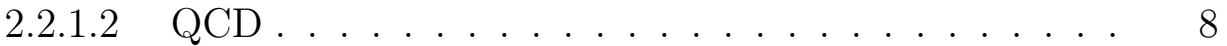

2.2.1.3 Electroweak (GWS) Theory and The Higgs Mechanism 9

2.2.2 Beyond The Standard Model . . . . . . . . . . . . . . . 13

2.3 Hidden-Valley Phenomenology . . . . . . . . . . . . . . . . 15

2.3.1 A Specific Hidden-Valley Model . . . . . . . . . . . . . . . . . 17

2.3.2 Experimental Constraints on Hidden-Valley Models . . . . . . 19

2.4 Overview of the Analysis . . . . . . . . . . . . . . . . 20 
3 Experimental Apparatus $\quad 26$

3.1 Units and Coordinates . . . . . . . . . . . . . 26

3.1 Luminosity . . . . . . . . . . . . . 26

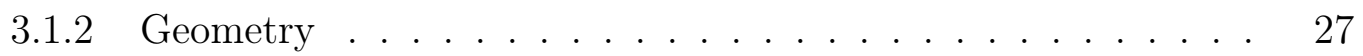

3.2 Physics Objects . . . . . . . . . . . . . . . . . . . 28

3.3 The Tevatron and Accelerator Chain . . . . . . . . . . . . . . 29

3.3.1 The Fermilab Accelerators . . . . . . . . . . . . . . . . . 30

3.3.2 Proton-Antiproton Collisions . . . . . . . . . . . . . 32

3.4 The DØ Detector . . . . . . . . . . . . . . . . 33

3.4.1 The Inner Detectors . . . . . . . . . . . . . . . . 33

3.4.1.1 The Luminosity Monitor . . . . . . . . . . . . 35

3.4.1.2 The Silicon Microstrip Tracker . . . . . . . . . . 36

3.4.1.3 The Central Fiber Tracker . . . . . . . . . . . 37

3.4 .2 The Calorimeter . . . . . . . . . . . . . . . 38

3.4.3 The Muon System . . . . . . . . . . . . . . . . 42

3.4.4 Triggering and Data Acquisition ............. 44

4 Reconstruction and Calibration $\quad 48$

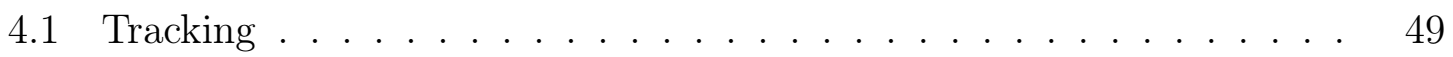

4.1 .1 Hit Clustering . . . . . . . . . . . . . . . . 49

4.1 .2 Track Finding . . . . . . . . . . . . . . . 50

4.1.2.1 Histogram Track Finding . . . . . . . . . . 51

4.1.2.2 Alternate Algorithm . . . . . . . . . . . 51

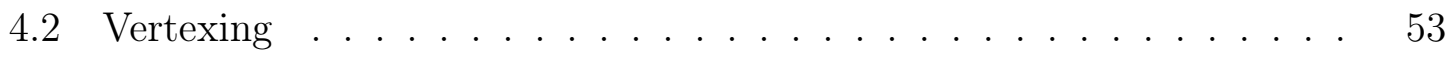

4.2.1 The Primary-Vertex ................ 54 
$4.2 .2 \quad$ Secondary-Vertices . . . . . . . . . . . . . . . . 55

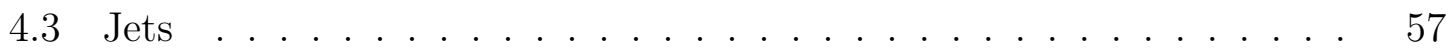

4.3 .1 Reconstruction . . . . . . . . . . . . . . . 59

4.3 .2 Jet Selection Criteria . . . . . . . . . . . . . . . . . 60

4.3 .3 Jet Energy Scale . . . . . . . . . . . . . . . . . . . . 61

4.4 Muons . . . . . . . . . . . . . . . . . . . . . . 62

5 Data and Simulation $\quad 64$

5.1 Data . . . . . . . . . . . . . . . . . . 64

5.2 Monte Carlo Techniques . . . . . . . . . . . . . . . 67

5.2.1 Background Monte-Carlo Samples . . . . . . . . . . . . . 68

5.2 .2 Background . . . . . . . . . . . . . . . . . 68

5.2 .3 Signal Modeling . . . . . . . . . . . . . . . . . . . . . . . . 69

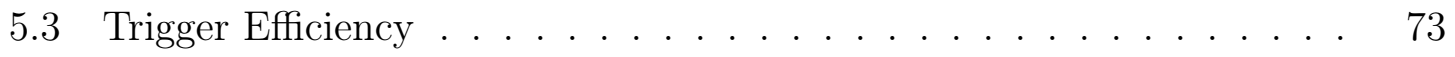

6 Analysis $\quad 84$

6.1 Discriminating Handles . . . . . . . . . . . . . . . . 85

6.2 Event Pre-Selection . . . . . . . . . . . . . . . . . . 86

6.3 Background Modeling . . . . . . . . . . . . . . . . . . 95

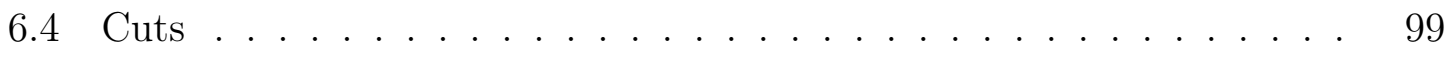

6.5 Limit Setting . . . . . . . . . . . . . . . . . . . . 104

6.5.1 The $C L_{S}$ Approach _ . . . . . . . . . . . . . . . 109

6.5.2 The Likelihood Ratio . . . . . . . . . . . . . . . . . . . . . 111

6.5.3 Confidence-Limit Results . . . . . . . . . . . . . . . . . . 112

6.6 Results . . . . . . . . . . . . . . . . . . . . . . . . . . . 114 
6.6.1 Systematic Uncertainties . . . . . . . . . . . . . . . 114

6.6.2 Material Interaction Cross Check . . . . . . . . . . . . . . . . 123

6.7 Summary . . . . . . . . . . . . . . . . . . . . 124

$\begin{array}{lr}\text { Bibliography } & 128\end{array}$

A Trigger Efficiency 133

A.1 Trigger Frequency . . . . . . . . . . . . . . . . . 133

A.2 File Locations . . . . . . . . . . . . . . . . . . . . . . . . . . . . 134

A.3 Trigger Efficiency Plots . . . . . . . . . . . . . . 135

$\begin{array}{ll}\text { B Comparing p17 and p20 Data } & 137\end{array}$

C Secondary-vertex Density Cuts 139

$\begin{array}{ll}\text { D Event Displays } & 142\end{array}$

$\begin{array}{ll}\text { E Candidate Events } & 147\end{array}$ 


\section{List of Figures}

1.1 Aerial view of the Fermilab accelerator complex. . . . . . . . . . . . . 4

2.1 Global fit to precision electroweak data showing the preferred mass for the Higgs boson. Yellow areas are excluded by experiments. . . . . . 14

2.2 Gauge couplings in the Standard Model. . . . . . . . . . . . . . . . . 15

2.3 A possible v-particle production mechanism. . . . . . . . . . . . 18

2.4 A possible Higgs decay through mixing. . . . . . . . . . . . . . . . . . 19

2.5 In this event, three v-hadrons are produced at the primary vertex (red circle), one of which decays in the tracker (blue circle). The secondaryvertex consists of two b-quark jets with their subsequent decays indicated by multiple tracks. . . . . . . . . . . . . . . . . .

2.6 A MC simulated Higgs decay in the X-Y plane. The dark-blue primary vertex is in the center within the beam pipe which is at a radius of 1.6 $\mathrm{cm}$. There are three secondary vertices represented by the red-orange circles. Blue lines represent the material of the SMT ladders. . . . . 
2.7 A MC simulated background event resulting from a material interaction. Six tracks are reconstructed to produce the secondary vertex which is located in a ladder of the SMT. . . . . . . . . . . . . .

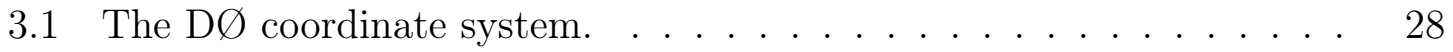

3.2 Integrated luminosity delivered by the Tevatron collider. . . . . . . 30

3.3 Fermilab's accelerator chain. . . . . . . . . . . . . . . . . . 31

3.4 The upgraded D $\varnothing$ detector for Run II. Protons enter from the left while antiprotons enter from the right. Collisions occur near the center of the detector. The innermost portion of the detector consists of the tracker which is contained within a solenoidal magnet. The calorimeter is in the central portion and the muon system, which contains an iron toroid magnet, comprises the outermost portion. . . . . . . . . . .

3.5 The silicon microstrip tracker geometry. Six barrels cover the central region (about $|\eta| \leq 1.1$ ) while the F-disks and H-disks cover the high $\eta$ region $(|\eta| \leq 3.0) \ldots \ldots \ldots \ldots \ldots \ldots \ldots \ldots \ldots \ldots$

3.6 Cross-sectional view of the inner detectors in the $\mathrm{x}-\mathrm{y}$ plane. . . . . . . 39

3.7 A 3D view of the calorimeter. The central and end-cap calorimeters are contained in separate cryostats. . . . . . . . . . . . . 40

3.8 A side view of the calorimeter. The calorimeter is segmented into cells of size in $\eta$ and $\phi$. In the electromagnetic and fine hadronic portion the cell size is $\eta \times \phi=0.1 \times 0.1$ while in the coarse hadronic portion the cell size is $\eta \times \phi=0.2 \times 0.2 \ldots \ldots \ldots \ldots \ldots \ldots$. . . . . . 41

3.9 Schematic view of the liquid argon gap and signal board unit cell for the calorimeter. . . . . . . . . . . . . . . . . 
3.10 The $\mathrm{D} \varnothing$ muon detector. The bottom of the detector (not shown) is not fully instrumented and contains holes for the support structure and wiring. The toroid magnet sits between the A and B layers. . . . . . 43

3.11 Overview of the $\mathrm{D} \varnothing$ trigger and data aquisition systems. . . . . . . . 44

3.12 The doublet (left) and singlet (right) schemes for the CTT triggers. The circles represent fibers and the solid line represents the actual track trajectory. . . . . . . . . . . . . . .

4.1 Example of the histogram track finding technique for a single $1.5 \mathrm{GeV}$ track coming from the center of the detector and producing 5 hits. Plot (a) shows the family of trajectories containing a given hit. Plot (b) shows the line in Hough transformed space. Plot (c) shows curves from different hits intersecting at one point corresponding to the track parameters. Plot $(\mathrm{d})$ shows the peak in the $(\rho, \phi)$ histogram which corresponds to the intersection of the lines in parameter space. . . .

4.2 A sketch of the evolution from the hard-scatter parton to a jet in the

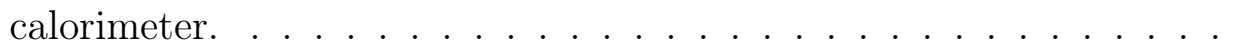

4.3 An illustration of soft radiation sensitivity in the cone jet algorithm. In this example, jet clustering begins around seed particles, shown here as arrows with length proportional to their energy. The presence of soft radiation between the two jets may cause a merging of the jets which would otherwise not occur. . . . . . . . . . . . . . . . 
5.1 A fit of the number of primary vertices to a Poisson distribution to estimate the luminosity of the dataset after requiring 3 or fewer primary vertices per event. Green is the fitted line and black is the data used in the analysis. The uncertainty includes both the standard luminosity uncertainty as well as the uncertainty from the fit. The average instantaneous luminosity in run $2 \mathrm{~b}$ (p20; on the right) was higher than in run $2 \mathrm{a}$ (p17; on the left) which produced more primary-vertices per event. As a result, more data was cut in p20 than in p17. The total integrated luminosity of the two samples is $2712 \mathrm{fb}^{-1}$. . . . . . .

5.2 Agreement between QCD MC and $b \bar{b}+X$ and $c \bar{c}+X$ MC. All samples are normalized to each other. The "cut0" pre-selection criteria requires that each SV must be reconstructed with $\chi^{2}<20$, have a track multiplicity greater than 4 and be located at a distance of at least $1.6 \mathrm{~cm}$ in the transverse plane. The cut0 pre-selection is discussed in section 6.2. 70

5.3 QCD MC after reweighting to run 2a (p17; on the left) and run 2b (p20; on the right) data. All samples are normalized to each other. The "cut0" pre-selection criterion is defined in section 6.2 . . . . . . . 71

5.4 QCD MC before and after reweighting to run 2a (p17; on the left) and run 2b (p20; on the right) data. All samples are normalized to each other. The "cut0" pre-selection criterion is defined in section 6.2. . .

5.5 A comparison of data collected with the selected single trigger and all triggers. Run $2 \mathrm{a}$ is on the left and Run $2 \mathrm{~b}$ on the right. The $m_{H}=120$ $\mathrm{GeV}, m_{H V}=15 \mathrm{GeV}, \mathrm{dl}=50 \mathrm{~mm}$ signal sample is shown to illustrate the location of signal events. . . . . . . . . . . . . 
5.6 The ratio of the number of events from the single trigger to all triggers as a function of leading muon $p_{T}$ and leading jet $p_{T}$. The Run $2 \mathrm{a}$ trigger ratio is on the left and the Run $2 \mathrm{~b}$ ratio is on the right. The $m_{H}=120 \mathrm{GeV}, m_{H V}=15 \mathrm{GeV}, \mathrm{dl}=50 \mathrm{~mm}$ signal sample is shown to illustrate the location of signal events. The black line is events collected by all the triggers, the red line is signal events, the green line is events collected by the single-trigger, and the line represented by the black cross section is the ratio of the number of all-trigger events to single-trigger events. . . . . . . . . . . . . . . .

5.7 Leading jet $p_{T}$ and overall trigger efficiency as a function of leading jet $p_{T}$ for three different signal samples. Plots on the left show the total p17 trigger efficiency while plots on the right show total p20 trigger efficiency. . . . . . . . . . . . . . . .

5.8 Leading jet $p_{T}$ and overall trigger efficiency as a function of leading jet $p_{T}$ for three different signal samples. Plots on the left show the total p17 trigger efficiency while plots on the right show total p20 trigger

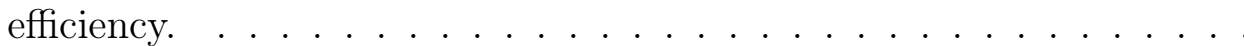

5.9 Leading jet $p_{T}$ and overall trigger efficiency as a function of leading jet $p_{T}$ for one signal sample. The plot on the left show the total p17 trigger efficiency while the plot on the right show total p20 trigger efficiency.

6.1 The vertex collinearity is defined as the cosine of the angle between the vector sum of the momentum of the decay products and the direction

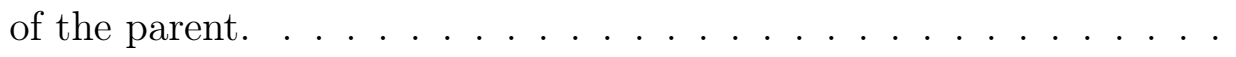


6.2 The secondary-vertex mass for three different signal hypotheses and background. Signal is normalized to QCD to illustrate the difference in shape. . . . . . . . . . . . . . . . . . .

6.3 The multiplicity of tracks attached to secondary-vertices for three different signal hypotheses and background. Signal is normalized to QCD to illustrate the difference in shape. . . . . . . . . . . . . . . . 88

6.4 The collinearity of tracks attached to secondary-vertices for three different signal hypotheses and background. Signal is normalized to QCD to illustrate the difference in shape. . . . . . . . . . . . . . .

6.5 Material map before and after cutting out areas of high SV density for the p17 and p20 data sets. Secondary-vertices with track multiplicity $\geq 3$ are used to generate the map. No other pre-selection criteria are required for events in this plot. . . . . . . . . . . . .

6.6 Material map of the $x y$-plane before and after cutting out areas of high SV density for the p17 and p20 data sets. Secondary-vertices with track multiplicity $\geq 3$ are used to generate the map. No other pre-selection criteria are required for events in this plot. . . . . . . .

6.7 The square root of the density of secondary-vertices in the $x y$-plane after the cut0 pre-selection. The dashed line indicates the location of

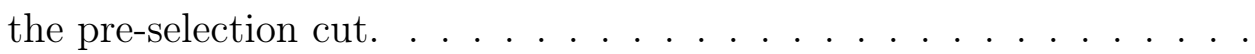

6.8 The square root of the density of secondary-vertices in the $x y$-plane after the cut0 pre-selection. The dashed line indicates the location of the pre-selection cut. . . . . . . . . . . . . . 
6.9 Comparison of discriminating variables for data (combined p17 and p20) with $\chi^{2}(S V)<20$ and data with $20<\chi^{2}(S V)<100$. No other cut beyond the $\chi^{2}$ cut is imposed on the events. . . . . . . . .

6.10 Secondary-vertex $\chi^{2}$ per degree of freedom, there were no pre-selection criteria placed on events in this plot. The dashed line at $\chi^{2}=20$ shows the location of the pre-selection cut. Data is combined p17 and p20. . 95

6.11 Two dimensional decay length. The dashed line at $1.6 \mathrm{~cm}$ shows the location of the pre-selection cut. . . . . . . . . . . .

$6.12 \Delta R$ between secondary-vertices after the cut0 pre-selection requirement for the p17 and p20 data sets. Two SVs per event are required. QCD is normalized to data. . . . . . . . . . . . .

6.13 The number of secondary-vertices per event for p17 and p20 secondary-

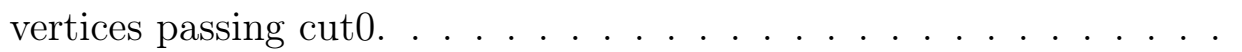

6.14 Comparing smeared and unsmeared QCD to run 2a (p17) data for SV mass and collinearity. The mass is smeared by a Gaussian with a sigma of $12 \mathrm{GeV}$ which is applied to $0.50 \%$ of the events. The collinearity is smeared by a Gaussian with a sigma of 0.10 , applied to $1.50 \%$ of the events. . . . . . . . . . . . . . . . . . 100

6.15 Comparing smeared and unsmeared QCD to run $2 \mathrm{~b}$ (p20) data for SV mass and collinearity. The mass is smeared by a Gaussian with a sigma of $12 \mathrm{GeV}$ which is applied to $1.25 \%$ of the events. The collinearity is smeared by a Gaussian with a sigma of 0.20 , applied to $1.75 \%$ of the events. . . . . . . . . . . . . . . . . . . . 101 
6.16 Secondary-vertex track multiplicity for p17 and p20 data and MC. SVs in this plot were required to have $\chi^{2}<20$ and $\mathrm{dl} 2 \mathrm{~d}>1.6 \mathrm{~cm} \mathrm{~cm}$ as discussed in section 6.2. QCD was normalized to data for multiplicities

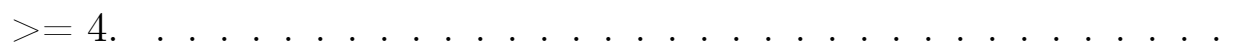

6.17 An example of the result of smoothing mass and collinearity with the Root function, TH1::Smooth(). . . . . . . . . . . . . . 103

6.18 The minimum of the mass of the two secondary-vertices. This plot shows the significance of making a cut on the signal $\left(m_{H}=200 \mathrm{GeV}, m_{H V}=\right.$ $15 \mathrm{GeV}, d l=50 \mathrm{~mm}$ ) and background MC. . . . . . . . . . . . 104

6.19 The minimum of the mass of the two secondary-vertices. This plot shows the significance of making a cut on the signal $\left(m_{H}=120 \mathrm{GeV}, m_{H V}=\right.$ $15 \mathrm{GeV}, d l=25 \mathrm{~mm}$ ) and background MC . . . . . . . . . . . 105

6.20 The minimum of the mass of the two secondary-vertices. This plot shows the significance of making a cut on the signal $\left(m_{H}=120 \mathrm{GeV}, m_{H V}=\right.$ $15 \mathrm{GeV}, d l=50 \mathrm{~mm}$ ) and background MC. . . . . . . . . . . . 105

6.21 The minimum of the mass of the two secondary-vertices. This plot shows the significance of making a cut on the signal $\left(m_{H}=120 \mathrm{GeV}, m_{H V}=\right.$ $15 \mathrm{GeV}, d l=100 \mathrm{~mm}$ ) and background MC. . . . . . . . . . . . 106

6.22 The minimum of the mass of the two secondary-vertices. This plot shows the significance of making a cut on the signal $\left(m_{H}=90 \mathrm{GeV}, m_{H V}=\right.$ $15 \mathrm{GeV}, d l=50 \mathrm{~mm}$ ) and background MC. . . . . . . . . . . 106

6.23 The maximum of the collinearity of the two secondary-vertices. This plot shows the significance of making a cut on the signal $\left(m_{H}=\right.$ $200 \mathrm{GeV}, m_{H V}=40 \mathrm{GeV}, d l=50 \mathrm{~mm}$ ) and background MC. . . . . 107 
6.24 The maximum of the collinearity of the two secondary-vertices. This plot shows the significance of making a cut on the signal $\left(m_{H}=\right.$ $\left.120 \mathrm{GeV}, m_{H V}=40 \mathrm{GeV}, d l=50 \mathrm{~mm}\right)$ and background MC . . . . . 107

6.25 The maximum of the collinearity of the two secondary-vertices. This plot shows the significance of making a cut on the signal $\left(m_{H}=\right.$ $90 \mathrm{GeV}, m_{H V}=40 \mathrm{GeV}, d l=50 \mathrm{~mm}$ ) and background MC. . . . . 108

6.26 Distribution of - $2 \mathrm{LnQ}$ for the background and signal+background hypotheses for 1 million $\mathrm{MC}$ pseudo-experiments. The observed value of $-2 \mathrm{LnQ}$ is indicated by the red line. In this example, $m_{H}=120$ $\mathrm{GeV}, m_{H V}=15 \mathrm{GeV}, \mathrm{dl}=50 \mathrm{~mm}$ is the signal. The area under the background distribution to the left of the observed $\mathrm{Q}$ is $1-C L_{b}$ and the area under the signal+background distribution to the right of the observed $\mathrm{Q}$ is $C L_{s+b}$. . . . . . . . . . . . . . . . . . . . . . 112

6.27 The minimum of the mass between the two secondary-vertices. This plot compares the data to the signal and background $\mathrm{MC}$ for three signal hypotheses for which mass is the optimal cut. . . . . . . . . . 115

6.28 The minimum of the mass between the two secondary-vertices. This plot compares the data to the signal and background $\mathrm{MC}$ for two signal hypotheses for which mass is the optimal cut. . . . . . . . . . . . 116

6.29 The maximum of the collinearity between the two secondary-vertices. This plot compares the data to the signal and background MC for two signal hypotheses for which collinearity is the optimal cut. . . . . . . 117

6.30 Observed and expected cross-sections as a function of Higgs boson mass.119 
6.31 Observed and expected cross-sections as a function of HV particle decay length. . . . . . . . . . . . . . . . . . . . . 120

6.32 Comparison of QCD to data after reweighting the QCD track multiplicity to match the data. This is the combined p17 and p20 data

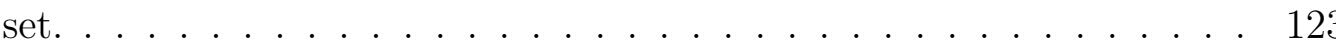

6.33 Secondary-vertex density in the $x y$-plane for data and QCD where QCD is normalized to data. The black crosses are the percent difference of remaining events between QCD and data if a cut is made at the given value. The dashed lines show the locations of the actual cuts that are

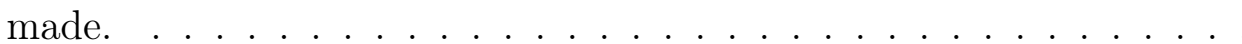

6.34 The SV density in the $x y$ plane for data secondary-vertices (two SVs per event). The red lines are data events resulting from the cuts used to search for the signal points while the black lines are the pre-selected data. . . . . . . . . . . . . . . . . . 125

6.35 The SV density in the $z r$ plane for data secondary-vertices (two SVs per event). The red lines are data events resulting from the cuts used to search for the signal points while the black lines are the pre-selected data. . . . . . . . . . . . . . . . . 126

A.1 The L1 and L2 efficiencies for the MU_2TRK3_L2M0 trigger with respect to an offline medium-quality, nseg $=3$ muon and a loose track. 135

A.2 The L1 efficiency of the MU_2TRK3_L2M0 trigger with respect to an offline good-jet. . . . . . . . . . . . . . . 135 
A.3 The L1 and L2 efficiencies for the MUJ5_MM0_2TK3_VX trigger with respect to an offline medium-quality, nseg $=3$ muon and a loose track. . . . . . . . . . . . . . . . . . . 136

A.4 The L1 and L2 efficiencies for the MUJ5_MM0_2TK3_VX trigger with respect to an offline good-jet. . . . . . . . . . . . . . . 136

B.1 Compare data collected during run 2a (p17) and run $2 \mathrm{~b}(\mathrm{p} 20) \ldots \ldots .138$

C.1 Secondary-vertex density map of the $x y$ and $z r$-planes before and after cutting out areas of high SV density for the p17 data set. Secondaryvertices with track multiplicity $\geq 3$ are used to generate the map. The cut0 pre-selection criteria is required for all events in these plots. . . . 140

C.2 Secondary-vertex density map of the $x y$ and $z r$-planes before and after cutting out areas of high SV density for the p20 data set. Secondaryvertices with track multiplicity $\geq 3$ are used to generate the map. The cut0 pre-selection criteria is required for all events in these plots. . . . 141

D.1 Event displays in $x y$ and $z r$-planes for p17 data in which two secondaryvertices had collinearity $<0.9977 \ldots \ldots \ldots \ldots$. . . . . . . 143

D.2 Event displays in $x y$-plane for p20 data in which two secondary-vertices had collinearity $<0.9977 . \ldots \ldots \ldots \ldots$. . . . . . . . . 143

D.3 Event displays in $x y$ and $z r$-planes for p17 data in which two secondaryvertices had mass $>2.5 \mathrm{GeV} . \ldots \ldots \ldots \ldots$. . . . . . . . 144

D.4 Event displays in $x y$ and $z r$-planes for p20 data in which two secondaryvertices had mass $>2.5 \mathrm{GeV} . \ldots \ldots \ldots \ldots$ 
D.5 Event displays in $x y$ and $z r$-planes for p20 data in which two secondaryvertices had mass $>2.5 \mathrm{GeV} . \ldots \ldots \ldots$ 


\section{List of Tables}

2.1 Fundamental particles in the Standard Model. . . . . . . . . . . . . . 6

5.1 The datasets used in this analysis. The integrated luminosity $\left(\int \mathcal{L}\right)$ is for "good quality" as defined by by the SMT, CFT, Calorimeter and Muon groups. ...................... 66

5.2 Summary of the signal hypotheses used in this analysis and number of events generated. . . . . . . . . . . . . . . .

5.3 The average trigger efficiency of each p20 trigger level for each signal sample. The p20 trigger is MUJ5_MM0_2TK3_VX. Events are required to pass the cut0 pre-selection (refer to table 6.1 for the definition

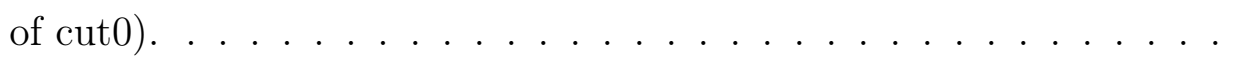

5.4 The average trigger efficiency of each p17 trigger level for each signal sample. The p17 trigger is MU_2TRK3_L2M0. Events are required to pass the cut0 pre-selection (refer to table 6.1 for the definition of

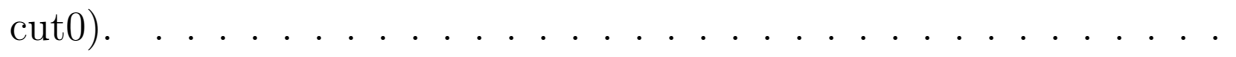


5.5 Average trigger efficiency for each signal sample including the jet turn on from figure 5.6. Events are required to pass the cut0 pre-selection (refer to table 6.1 for the definition of cut0). . . . . . . . .

6.1 Summary of all pre-selection cuts. The "p17" and "p20" columns indicate the number of data events passing each consecutive cut. Each event must contain at least one SV per event which passes the given pre-selection requirement as well as all of the previous requirements in the table. The $x y$ material density cut requires that events must have at least one SV with $\sqrt{\text { xydensity }}<11$ for p17 and $\sqrt{\text { xydensity }}<15$ for p20. The $z r$ material density cut requires that events must have at least one SV with $\sqrt{z r \text { density }}<40$ for p17 and $\sqrt{z r \text { density }}<59$ for p20. The $\Delta R$ cut only applies when there are two or more secondaryvertices in an event. . . . . . . . . . . . . . . . . .

6.2 The values of $C L_{s} @ 95 \%$ CL resulting from the top_statistics software package. The inputs come from section 6.4. . . . . . . . . . . . . 113

6.3 Summary of the cut-flow which is optimized for the signal hypotheses $\left(m_{H}=120, m_{H V}=15, d l=50 \mathrm{~mm}\right),\left(m_{H}=120, m_{H V}=15, d l=\right.$ $50 \mathrm{~mm})$ and $\left(m_{H}=90, m_{H V}=15, d l=50 \mathrm{~mm}\right)$. QCD is normalized to data after the pre-selection. . . . . . . . . . . . . . 118

6.4 Summary of the cut-flow which is optimized for the signal hypotheses $\left(m_{H}=120, m_{H V}=15, d l=100 m m\right)$ and $\left(m_{H}=200, m_{H V}=15, d l=\right.$ $50 \mathrm{~mm}$ ). QCD is normalized to data after the pre-selection. . . . . . . 118 
6.5 Summary of the cut-flow which is optimized for the signal hypotheses $\left(m_{H}=120, m_{H V}=40, d l=50 m m\right)$ and $\left(m_{H}=200, m_{H V}=40, d l=\right.$ $50 \mathrm{~mm}$ ). QCD is normalized to data after the pre-selection. . . . . . . 120

6.6 Summary of results for six signals hypotheses. Data from p17 and p20 are combined. . . . . . . . . . . . . . . . . 121

6.7 Summary of results for the last two signal hypotheses. Data from p17 and p20 are combined. . . . . . . . . . . . . . . . . . 122

6.8 Summary of the systematic uncertainties. . . . . . . . . . . . . 123

A.1 Run 2a trigger frequency for the most frequently used triggers . . . . 133

A.2 Run $2 \mathrm{~b}$ trigger frequency for the most frequently used triggers . . . . 134

E.1 Candidate events that pass the pre-selection criteria and maximum collinearity $<0.9977 \ldots \ldots \ldots \ldots$. . . . . . . . . . . . 148

E.2 Candidate events that pass the pre-selection criteria and minimum

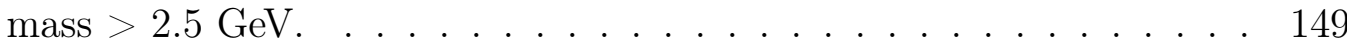




\section{Acknowledgements}

To Greg; your life was an inspiration. I would like to thank my advisor, Gustaaf Brooijmans, for providing fresh ideas at difficult junctures and reviewing my work. Without his help, none of this would have been possible. I would also like to acknowledge Andy Haas, whose inspired approach always produced fascinating new ideas, including the idea for this analysis. Thanks to Thomas Gadfort, for patiently explaining many details of $\mathrm{D} \varnothing$ and demanding more rigor from me. Thanks to Hal Evans for guiding my early hardware work on DØ. Ron McNabb was an independent ear and a faithful friend; spending many long hours polishing the presentation of my work. Finally, thanks to everyone at D $\varnothing$ and especially the New Phenomena group for providing reviews and useful advice. 


\section{Chapter 1}

\section{Introduction}

Everything that we see around us is composed of fundamental particles. The science

of particle physics seeks to describe the precise nature of these fundamental particles and the mechanism by which they interact with each other. What follows is a brief historical motivation for the present work and an outline of the thesis.

\subsection{Historical Introduction}

Modern particle physics was born in 1897 when JJ Thompson discovered the electron and measured its charge to mass ratio by bending a beam of accelerated electrons in a magnetic field. Nineteen-hundred marked the year that Max Planck identified the photon as the quantum of electromagnetic radiation and in 1905 Einstein used it to explain the photo-electric effect; the process by which electrons are ejected from an illuminated metallic surface. Ernest Rutherford discovered the atomic structure of ordinary matter by passing alpha particles through gold foil and noting that most particles were not deflected at all, while a few were deflected at very large angles. 
The result of his 1909 experiment could not be explained by the prevailing "plum pudding" model, but could be explained if a heavy nucleus were surrounded by a very low density of electrons. Dirac's treatment of relativist quantum mechanics gave rise to anti-particles and the anti-electron, or positron, was subsequently discovered by Carl Anderson in 1932. Wolfgang Pauli proposed the existence of the neutrino to solve the problem of energy conservation in beta decay. James Chadwick discovered the neutron in 1932, and in 1934 mesons were proposed by Hideki Yukawa to explain inter-nuclear forces. By 1947 Kaons were discovered and with them, a whole new class of "strange" particles. Hundreds of new and, seemingly, disparate particles were discovered in the following years. Is there a single theory that can bring all of these varied particles and their interactions together? The solution is the Standard Model.

The Standard Model of particle physics was developed in the 1960's and 1970's and it explains all known particle interactions in term of quantum fields (with the exception of gravity which will be neglected from now on). Although the Standard Model has been immensely successful, it demands the existence of one more field; the Higgs boson field, which has not yet been observed. The Standard Model also contains certain features which point to a new theory and the eventual observation of the Higgs boson may be intricately intertwined with the motivation for this new theory. For these reasons, discovering the Higgs boson is one of the most important activities of current particle physics research and is the impetus behind the present analysis. 


\subsection{Analysis Introduction}

The subject of this thesis is a novel search for long-lived particles that travel at least $1.6 \mathrm{~cm}$ in the lab's frame-of-reference before decaying to heavy quarks producing a highly displaced vertex. Particles of this nature may arise as a result of a hidden physics-sector. This sector, or "hidden-valley" as it is known in the literature, is energetically inaccessible to the SM at everyday energies and even at most highenergy experiments. However, it may be possible that the hidden-valley communicates with the SM through a particle, such as the Higgs boson, that could be produced at energies which are available to the Fermilab Tevatron Collider (Tevatron). The Tevatron is located at Fermilab outside of Chicago, IL. In order to produce the kinds of particles which are currently being explored, modern particle physics experiments are extraordinarily large and Fermilab is no exception. Figure 1.1 shows the Fermilab accelerator complex. The Tevatron is the world's highest energy collider, consisting of a superconducting ring that brings protons and antiprotons together for collisions at a center-of-mass energy of $1.96 \mathrm{TeV}$, an energy which is more than a thousand times the rest mass of a proton. There are two collision points at the Tevatron which are the locations of two large detectors that record information about the collisions. This analysis uses data collected by $\mathrm{D} \varnothing$, one of the two detectors on the Tevatron ring. One of the fundamental tenets of quantum mechanics is that any physical process that can happen, will happen with some probability. Therefore, we can maximize the chance of observing a rare physical process (such as the production of a hidden-valley particle) by colliding a large number of protons and antiprotons and analyzing as much of the data as possible. 


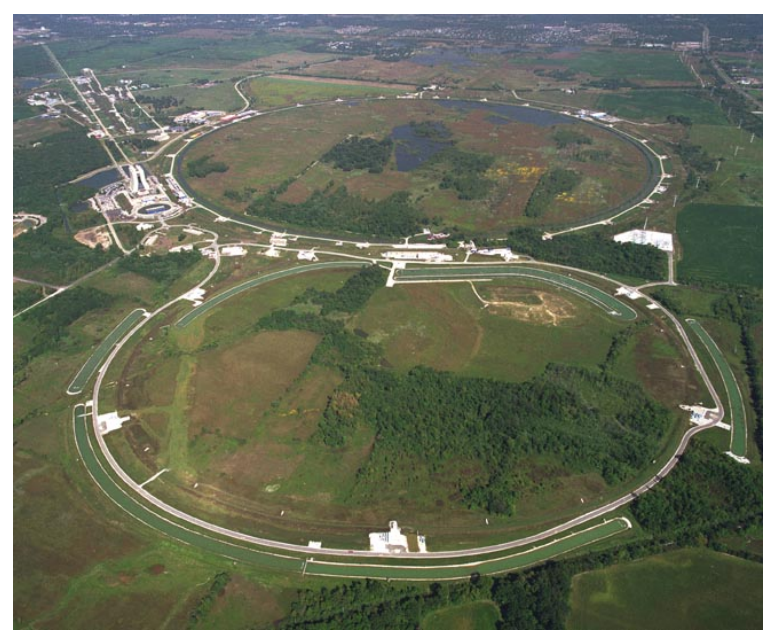

Figure 1.1: Aerial view of the Fermilab accelerator complex.

\subsection{Outline}

This thesis is divided into chapters as follows. Chapter 2 gives an introduction to the theory of the Standard Model and certain new particles which may exist as extensions to the Standard Model (or in any new theory that may replace the Standard Model). In order to facilitate a better understanding of why certain methods are employed, the chapter also gives an overview of the analysis which will be used to observe or exclude the existence of these particles. Chapter 3 contains a discussion of the experimental apparatus while the method of reconstruction and calibration is presented in chapter 4. Data and simulated data are discussed in chapter 5. Finally, chapter 6 presents the full analysis and its results. 


\section{Chapter 2}

\section{Theory and Experimental Introduction}

\subsection{Units}

Unless other units are explicitly stated, this thesis will utilize the so-called "natural units" in which $\hbar=c=1$. In this scheme mass, energy and momentum all have the same units of energy, usually measured in electron-volts or $e V$ where $1 \mathrm{eV}=$ $1.602 \times 10^{-19}$ Joules. In natural units, time and distance are both expressed in inverse energy while velocity is dimensionless. However, in these cases standard MKS units will usually be explicitly indicated to avoid confusion. For example, distances are often given in fermis where $1 \mathrm{fm}=10^{-15} \mathrm{~m}$ and cross-sections are given in barns where $1 \mathrm{~b}=10^{-28} \mathrm{~m}^{2}$. 


\begin{tabular}{|c|c|c|c|c|}
\hline & \multicolumn{3}{|c|}{$\begin{array}{c}\text { Fermion } \\
\text { Generations }\end{array}$} & \multirow{2}{*}{$\begin{array}{c}\text { vector } \\
\text { boson } \\
\text { force } \\
\text { carriers }\end{array}$} \\
\hline & I & II & III & \\
\hline \multirow{2}{*}{ quarks } & $\mathrm{u}$ & c & $\mathrm{t}$ & $\gamma$ \\
\hline & $\mathrm{d}$ & $\mathrm{s}$ & $\mathrm{b}$ & $\mathrm{g}$ \\
\hline \multirow{2}{*}{ leptons } & $\bar{e}$ & $\mu$ & $\tau$ & $W^{ \pm}$ \\
\hline & $\nu_{e}$ & $\nu_{\mu}$ & $\nu_{\tau}$ & $\mathrm{Z}$ \\
\hline
\end{tabular}

Table 2.1: Fundamental particles in the Standard Model.

\subsection{The Standard Model}

The Standard Model (SM) of particle physics is a theory of quantum fields in which particles are seen as excitations of the field. It is relativistic and quantum mechanical; valid at high relative velocity and short distances. The following discussion is based on references $[1,2,3]$. The Standard Model can be grouped by particle type as shown in table 2.1. The most general distinction is between the fermions which posses intrinsic spin $-\frac{1}{2}$ and the vector bosons which posses intrinsic spin -1 . Fermions are the constituents of matter and can be further subdivided into three generations while the vector bosons are the force carriers and mediate the interactions between particles. The SM contains three fundamental interactions: the strong described by quantum chromodynamics (QCD) theory, the electromagnetic described by quantum electrodynamics (QED) theory and the weak described by Glashow-Weinberg-Salam (GWS) theory. Each of these interactions are mediated by the vector bosons: the gluon or g (QCD), the photon or $\gamma(\mathrm{QED})$ and the $W^{ \pm}$and $Z$ bosons (GWS). Gravity is treated separately by General Relativity and not included in the SM. 


\subsubsection{Symmetries and Gauge Theories}

Symmetries play an important role in the Standard Model. According to Noether's theorem every symmetry implies a conservation law and every conservation law implies a corresponding symmetry. For example, rotational invariance gives rise to conservation of angular momentum and time translation invariance gives rise to energy conservation. All three interactions described by the SM are a result of a type of symmetry, known as local gauge invariance. This is an internal symmetry with respect to a group of parameters which remains invariant at all points in space and time under phase rotations, or changes of gauge. The change, $\psi \rightarrow e^{i \theta(x)} \psi$ is an example of a local gauge transformation while, $\psi \rightarrow e^{i \theta} \psi$ is a global gauge transformation. In response to Albert Einstein's geometric formulation of gravity, Hermann Weyl attempted to incorporate electromagnetism into geometry through the concept of a space-time dependent (local) scale transformation. Although initially unsuccessful, the term gauge is due to Weyl's original formulation.

\subsubsection{The QED Lagrangian}

The field equation for spin $-\frac{1}{2}$ particles is given by the Dirac Lagrangian which has global symmetry,

$$
\mathcal{L}=\bar{\psi}\left(i \gamma^{\mu} \partial_{\mu}-m\right) \psi
$$

where $\gamma^{\mu}$ are the Dirac matrices. From the global symmetry, Noether's theorem gives the conserved quantity, $J^{\mu}=e \bar{\psi} \gamma^{\mu} \psi$ which is simply electrical current density. We can impose local gauge invariance by making the replacement $\partial_{\mu} \rightarrow \mathcal{D}_{\mu} \equiv \partial_{\mu}+i e A_{\mu}$ where $\mathcal{D}_{\mu}$ is the covariant derivative and $A_{\mu}$ is a gauge field that transforms as, $A_{\mu} \rightarrow$ 
$A_{\mu}+\frac{1}{e} \partial_{\mu} \alpha(x)$. Then the Lagrangian becomes,

$$
\mathcal{L}=\bar{\psi}\left(i \gamma^{\mu} \mathcal{D}_{\mu}-m\right) \psi
$$

In order to make the gauge field a true dynamical quantity, we need to add a gaugeinvariant term, the simplest of which is, $-\frac{1}{4} F_{\mu \nu} F^{\mu \nu}$ where $F_{\mu \nu}=\partial_{\mu} A_{\nu}-\partial_{\nu} A_{\mu}$. How-

ever, the gauge field $A_{\mu}$ is required to be massless since $A_{\mu} A^{\nu}$ is not gauge invariant. We then arrive at the QED Lagrangian, were $A_{\mu}$ is the field equation of a photon,

$$
\mathcal{L}=\bar{\psi}\left(i \gamma^{\mu} \mathcal{D}_{\mu}-m\right) \psi-\frac{1}{4} F_{\mu \nu} F^{\mu \nu} .
$$

This particular form of symmetry is an example of a $U(1)$ symmetry since the local gauge transformation may be thought of as multiplying the field by a $1 \times 1$ unitary matrix, $\psi \rightarrow U \psi$ where $U^{\dagger} U=1$ implying that the matrices commute.

\subsubsection{QCD}

Other conservation laws can also be constructed by considering symmetries and the concept of local gauge invariance can be extended to groups whose members do not commute or, non-Abelian groups. In the preceding, $\psi$ is a two component spinor but one could require local gauge invariance of a more complicated field such as the three component color model of quarks. From this, the gauge theory of QCD can be derived and shown to be an $\mathrm{SU}(3)$ gauge symmetry (ie. the group of unitary matrices of determinant 1).

An important property of QCD that will figure prominently in the present work is confinement. As the separation distance between quarks increases, the energy 
density increases until there is enough energy to create quark-antiquark pairs from the vacuum. Thus, single quarks are never observed and only color singlets are observed. Another property of QCD is asymptotic freedom. The strength of QCD interactions goes down with increasing energy and, hence, at very high energies, quarks or gluons behave almost like free particles within hadrons. As a consequence, quarks and gluons are often generically called partons at high energy colliders such as the Tevatron where collisions appear to occur between the constituent partons rather than the hadrons.

\subsubsection{Electroweak (GWS) Theory and The Higgs Mechanism}

The weak interaction has always been something of a special case; unlike QCD and QED, the weak force is short-range necessitating a massive mediator. The weak interaction was first observed in $\beta$ decay for which Pauli postulated the existence of the neutrino to save conservation of energy. Prior to the advent of gauge theories, Enrico Fermi proposed his four-fermion interaction model, which is valid at low energies, to describe $\beta$ decay. In $1956 \mathrm{C}$. S. Wu conducted an experiment which measured the direction of electron emission from $C o^{60}$ decay and found that parity was not respected by the weak interaction. This was fixed by introducing the V-A Lagrangian; one part of the Lagrangian transforms as a vector and another part, which is subtracted, transforms as an axial-vector. The addition of a vector and an axial-vector must necessarily violate parity conservation. However, an additional problem arose in 1964 when weak decays in the K-meson system were found to violate charge conjugation and parity, or $\mathrm{CP}$, for which no natural solution existed. Additionally, the fourfermion theory violates unitarity and is not renormalizable.

The GWS theory unifies the weak and the electromagnetic interactions in a local 
gauge theory that has an $S U(2)_{L} \otimes U(1)_{Y}$ symmetry. However, as we have seen, gauge theories produce massless gauge bosons which is not what is observed in weak interactions and if masses are manually inserted, then the theory is not renormalizable. If, however, the underlying $S U(2)_{L} \otimes U(1)_{Y}$ gauge symmetry is spontaneously broken by the introduction of a massive scalar, then the correct spectrum of gauge bosons emerges. The massive scalar field is called the Higgs boson field and interactions with it produce the observed massive $W^{ \pm}$and $Z$ bosons.

Before electroweak symmetry breaking, the Lagrangian is given by,

$$
\mathcal{L}_{E W}=\mathcal{L}_{g}+\mathcal{L}_{f}+\mathcal{L}_{h}+\mathcal{L}_{y}
$$

where the $\mathrm{g}$ term describes the $\mathrm{SU}(2)$ and $\mathrm{U}(1)$ gauge-field tensors, the $\mathrm{f}$ term gives the couplings between fermions, the $\mathrm{h}$ term describes the Higgs field and the y term gives the Yukawa couplings between fermions and scalars. To see how spontaneous symmetry breaking can produce massive gauge bosons, we look at the Higgs Lagrangian which is given by,

$$
\begin{gathered}
\mathcal{L}_{h}=\left(D_{\mu} \Phi\right)^{\dagger}\left(D_{\mu} \Phi\right)-V(\Phi) \\
V(\Phi)=-\mu^{2} \Phi^{\dagger} \Phi+\lambda\left(\Phi^{\dagger} \Phi\right)^{2} .
\end{gathered}
$$

and using the covariant derivative,

$$
D_{\mu} \Phi=\left(\partial_{\mu}-\frac{i}{2} g \boldsymbol{\tau} \cdot \boldsymbol{A}_{\boldsymbol{\mu}}-\frac{i}{2} g^{\prime} B_{\mu}\right) \Phi
$$

where $A_{\mu}$ and $B_{\mu}$ are are, respectively, the $\mathrm{SU}(2)$ and $\mathrm{U}(1)$ gauge bosons and $\Phi$ is an 
$\mathrm{SU}(2)$ complex doublet of scalar particles,

$$
\Phi=\left(\begin{array}{l}
\phi^{+} \\
\phi^{-}
\end{array}\right)
$$

If $\mu$ and $\lambda$ are both positive, then the vacuum expectation value (VEV) of $\Phi$ is no longer zero,

$$
\langle\Phi\rangle_{0}=\frac{1}{\sqrt{2}}\left(\begin{array}{l}
0 \\
v
\end{array}\right)
$$

and the lowest potential energy is, $\left\langle\Phi^{\dagger} \Phi\right\rangle_{0}=\frac{v^{2}}{2}$ where $v=\sqrt{\frac{\mu^{2}}{\lambda}}$. Using polar variables for the scalar fields,

$$
\Phi(x)=U^{-1}(\xi)\left(\begin{array}{c}
0 \\
\frac{v+\eta(x)}{\sqrt{2}}
\end{array}\right)
$$

with $U(\xi)=\exp (i \boldsymbol{\xi}(\boldsymbol{x}) \cdot \boldsymbol{\tau} / v)$. The original complex fields $\phi^{+}(x)$ and $\phi^{-}(x)$ are now expressed in terms of four real fields with zero VEV: $\xi(x)$ which is identified with the Goldstone bosons and $\eta(x)$ which is identified with the Higgs boson.

All of the basic machinery is now in place and we need to make a gauge transformation to the unitary gauge in order to observe the particle spectra. The result of the transformation is given by,

$$
\Phi^{\prime}=U(\xi) \Phi=\left(\begin{array}{c}
0 \\
\frac{v+\eta(x)}{\sqrt{2}}
\end{array}\right)
$$

In the new gauge, equations 2.2 and 2.3 become,

$$
\begin{gathered}
D_{\mu} \Phi^{\prime}=\left(\partial_{\mu}-i \frac{g}{2} \boldsymbol{\tau} \cdot \boldsymbol{A}_{\boldsymbol{\mu}}^{\prime}-i \frac{g^{\prime}}{2} B_{\mu}^{\prime}\right)\left[\frac{v+\eta(x)}{\sqrt{2}}\right] \chi \\
V\left(\Phi^{\prime}\right)=\mu^{2} \eta^{2}+\lambda v \eta^{3}+\frac{\lambda}{4} \eta^{4}
\end{gathered}
$$


with $\chi=\left(\begin{array}{l}0 \\ 1\end{array}\right)$. The scalar Higgs boson's mass is then $m_{\eta}=\sqrt{2} \mu$. The vector boson masses are contained in the $\left(D_{\mu} \Phi^{\prime}\right)^{2}$ portion of the Lagrangian (equation 2.1) which, after carrying out the dot products, is given by,

$$
\begin{gathered}
\mathcal{L}_{V B}=\frac{v^{2}}{8} \chi\left\{g^{2}\left[\left(A_{\mu}^{\prime 1}\right)^{2}+\left(A_{\mu}^{\prime 2}\right)^{2}\right]+\left(g A_{\mu}^{\prime 3}-g^{\prime} B_{\mu}^{\prime}\right)^{2}\right\} \\
\equiv M_{W}^{2} W_{\mu}^{+} W^{-\mu}+\frac{1}{2} M_{Z}^{2} Z_{\mu} Z^{\mu}
\end{gathered}
$$

and we make the following identifications:

$$
\begin{gathered}
W_{\mu}^{ \pm}=\frac{1}{\sqrt{2}}\left(A_{\mu}^{1} \mp i A_{\mu}^{2}\right) \text { with mass } m_{W}=g \frac{v}{2} . \\
Z_{\mu}^{0}=\frac{1}{\sqrt{g^{2}+g^{\prime 2}}}\left(g A_{\mu}^{3}-g^{\prime} B_{\mu}\right) \text { with mass } m_{Z}=\sqrt{g^{2}+g^{\prime 2}} \frac{v}{2} . \\
A_{\mu}=\frac{1}{\sqrt{g^{2}+g^{\prime 2}}}\left(g^{\prime} A_{\mu}^{3}+g B_{\mu}\right) \text { with mass } m_{A}=0 .
\end{gathered}
$$

The charged $W_{\mu}^{ \pm}$and the neutral $Z_{\mu}$ are the massive gauge bosons of the weak interaction while the $A_{\mu}$ is the massless photon. Alternatively, the four gauge fields can be expressed in term of the Weinberg weak mixing angle, $\theta_{W} \equiv \arctan \left(g^{\prime} / g\right)$, in which case the masses of the bosons can be related by, $m_{W}=m_{Z} \cos \theta_{W}$. Currently, the measured value of the $\mathrm{W}$ mass is $80.398 \pm 0.025 \mathrm{GeV}$ and the measured value of the $\mathrm{Z}$ mass is $91.1876 \pm 0.0021 \mathrm{GeV}[4]$.

To summarize, the electromagnetic interaction and the weak interaction are found to result from a local gauge $S U(2)_{L} \otimes U(1)_{Y}$ theory known as the Glashow-WeinbergSalam (GWS) model. The underlying symmetry is broken by the introduction of a massive scalar field, the Higgs boson field. By a careful choice of gauge, two massive 
and one massless gauge field emerges, corresponding to the $W$ and $Z$ boson and the photon. The theory is also renormalizable. In 1971 't Hooft proved that all gauge theories, even those with spontaneous symmetry breaking, are renormalizable as long as certain anomalies cancel. With the observation of both the $W$ and $Z$, all that remains to fully confirm the GWS theory is to discover the Higgs boson. Direct searches at LEP have excluded at 95\% C.L. a SM Higgs boson for $m_{H}<114.4 \mathrm{GeV}$ [5] and the Tevatron has recently excluded at $95 \%$ C.L. $m_{H}=160-170 \mathrm{GeV}$ [6]. The $m_{H}<114.4 \mathrm{GeV}$ LEP limit only excludes SM Higgs decays $(h \rightarrow b \bar{b})$ while the most general LEP limit is $M_{H}>82 \mathrm{GeV}$ coming from a Higgs radiating off a $\mathrm{Z}$ boson [7].

\subsubsection{Beyond The Standard Model}

Despite the tremendous success of the Standard Model, there are still a few theoretical prejudices against it. The first is the constraint on the Higgs mass from the experiments at the Large Electron-Positron Collider (LEP) [5] which excluded much of the preferred SM prediction. LEP collided electrons and positrons at center-ofmass energies between 91 and $209 \mathrm{GeV}$ and primarily looked for Higgs production in association with a $Z$ boson and a subsequent Higgs decay to $b \bar{b}$ quark pairs via, $e^{+} e^{-} \rightarrow(H \rightarrow b \bar{b}) Z$. No Higgs boson candidates were observed which requires that the Higgs mass be greater than $114.4 \mathrm{GeV}$ [8] at 95\% CL. The existence of the Higgs boson is needed to explain the massive vector bosons of the weak interaction as demonstrated in section 2.2.1.3 and, although the SM is not able to predict the mass of the Higgs boson, SM particles are subject to radiative corrections from interactions with the Higgs field. A fit can be performed of all of the precision measurements of the electroweak parameters as shown in figure 2.1. Currently these measurements 


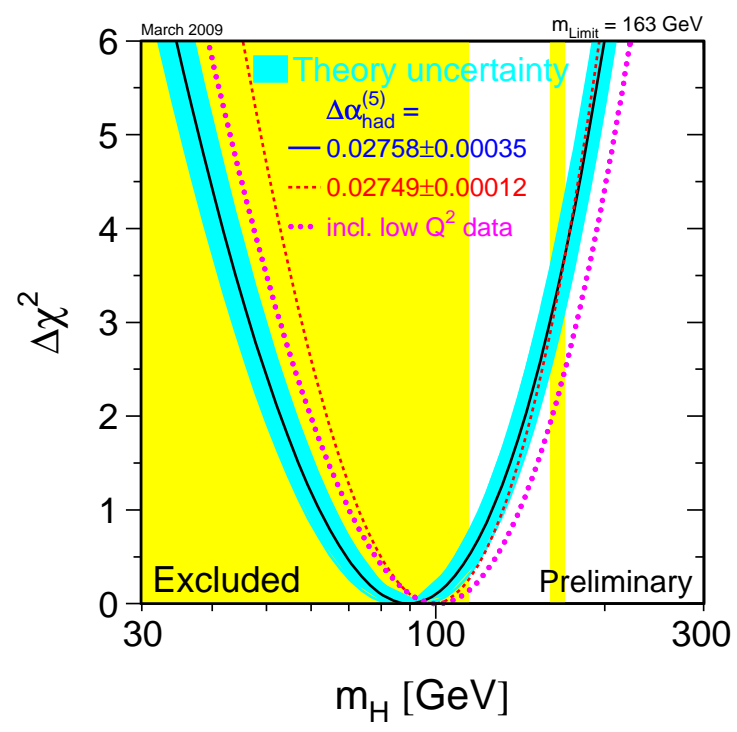

Figure 2.1: Global fit to precision electroweak data showing the preferred mass for the Higgs boson. Yellow areas are excluded by experiments [9].

predict that the SM Higgs boson mass is $m_{H}=90_{-27}^{+36} \mathrm{GeV}$ and constrained at $95 \%$ CL to a mass $m_{H}<191 \mathrm{GeV}$. Consequently, LEP has ruled out much of the most preferred mass as determined from electroweak fits, although of course, this does not rule out the SM Higgs.

The second issue is the unification of the gauge symmetries into a larger gauge group. The trend in physics has been a gradual unification of the fundamental interactions; Maxwell's theory of electromagnetism and the GWS electroweak theory, for example. Each of the fundamental interactions in the SM are characterized by coupling strengths which are energy dependent. If unification is to occur, the strengths must all be identical at some high energy, however, this does not occur in the SM as demonstrated in figure 2.2. It is hoped that in a future theory, the forces will unify.

In the SM, the Higgs boson mass would naturally tend to a very high mass value 


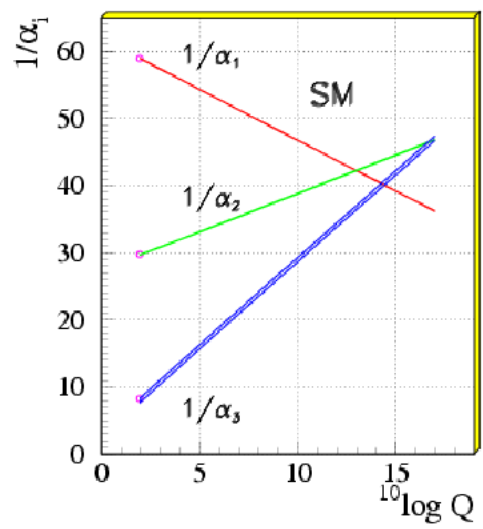

Figure 2.2: Gauge coupling evolutions in the Standard Model [4].

where gravity becomes important, yet all measurements indicate a light Higgs. The Higgs field can undergo radiative corrections and these corrections would tend to push the Higgs mass to the Planck scale if not for fine-tuned cancellations. A future theory should explain the light Higgs in a more natural way.

The SM has 19 input parameters which must be experimentally measured. This seems rather ad hoc and any future theory should have fewer input parameters.

Finally, there are the cosmological problems: there is no explanation in the SM for the matter-antimatter asymmetry of the universe, the SM has no dark matter candidate and it is also impossible to incorporate gravity into the SM.

\subsection{Hidden-Valley Phenomenology}

It is important for experimentalists to employ novel techniques to find the Higgs boson in order to explore all possible theoretical models and overcome inherent experimental constraints. Section 2.2.2 concluded that, despite the tremendous success of the Standard Model, it cannot be a complete description of nature. In particular, the fact 
that the Higgs boson has not been observed and much of the most favored mass has been excluded, hints that the Higgs may not be a Standard Model Higgs or it may not directly interact with Standard Model particles, in which case current experimental techniques, even at higher energy and higher luminosity accelerators, might never see the Higgs. Furthermore, traditional Higgs searches, such as $p \bar{p} \rightarrow W\left(\rightarrow l \nu_{l}\right) H(\rightarrow$ $b \bar{b})$ face tremendous background from light-jet misidentification as well as top-quark production and $W$ production in association with a b or c-quark [10]. Advanced techniques such as neural networks are employed to overcome the background and extract a signal, however cross section limits are still substantially larger than SM expectation in this channel [10]. If the Higgs production cross section is close to the SM prediction and has a mass of $m_{H}=120-200 \mathrm{GeV}$, then the Tevatron has already produced 2000-4000 Higgs bosons. If the Higgs decays decays to invisible particles with a moderate lifetime, leading to vertices displaced macroscopically far away from the $p \bar{p}$ interaction point, the signature could be spectacular enough that few events would be necessary to claim an observation, or least evidence, of the Higgs boson. In this way, the Tevatron may have a chance at discovering the Higgs before it is superseded by the Large Hadron Collider.

Although we consider a specific class of models which may give rise to highly displaced vertices, the analysis presented in this thesis is of a general nature and the results are applicable to any model proposing a long-lived state which can decay to heavy quarks. Other models with potentially long-lived states have been proposed, such as those found in reference [11]. Despite a wide array of proposed models, few searches which are sensitive to hidden-valley models have been undertaken, other than $[12,13,14,15]$, and some of these results have been controversial [16]. 


\subsubsection{A Specific Hidden-Valley Model}

Reference [17] predicts a confining gauge group weakly-coupled to the standard model, known as a "hidden-valley" (HV). The HV would be like a mirror of SM QCD but energetically inaccessible to LEP experiments while possibly accessible at Tevatron energies. In these models a particle which is charged in both the SM gauge group, $G_{S M}$, and in the HV gauge group, $G_{H V}$, could act as a communicator. Candidates for this role include the Higgs boson, the $Z^{\prime}$, the lightest standard model superpartner, a heavy sterile neutrino, or some other loop of heavy particles carrying both $G_{S M}$ and $G_{H V}$ charges.

For concreteness, consider the simplest example hidden-sector from [17] which adds a broken $S U(2) \times U(1)$ gauge group that is similar to SM QCD. The $S U(2)$ interaction confines at the scale, $1 \mathrm{GeV}<\Lambda_{v}<1 \mathrm{TeV}$. The $U(1)$ interaction is broken by a scalar expectation value $\langle\phi\rangle$ giving a mass of $1-6 \mathrm{TeV}$ to the gauge mediator, the $Z^{\prime}$. Consider a hidden-valley that consists of two quarks (v-quarks or $Q$ ), labeled $\mathrm{U}$ and $\mathrm{C}$ which hadronize due to confinement. Both quarks have a mass much less than the confinement scale, $\Lambda_{v}$. In this regime, an approximate v-isospin between $\mathrm{U}$ and $\mathrm{C}$ controls the spectrum and and the v-hadrons are labeled $\pi_{v}^{ \pm}$and $\pi_{v}^{0}$. Note that these v-hadrons are electrically neutral. All of the v-hadrons are stable and invisible to the SM except for $\pi_{v}^{0}$ which has the wavefunction $U \bar{U}-C \bar{C}$ and decays via $Q \bar{Q} \rightarrow Z^{\prime} \rightarrow f \bar{f}$ where $f$ is any SM fermion. Since v-particles are uncharged in $G_{S M}$, they would be very long lived. If $2 m_{b}<m_{\pi_{v}}<m_{t}$ the dominant decay is $\pi_{v}^{0} \rightarrow b \bar{b}$, due to helicity suppression ${ }^{1}$ and if $m_{\pi_{v}} \ll m_{Z}$ then the decay width is given

\footnotetext{
${ }^{1}$ The HV pion decays preferentially to heavy particles for the same helicity considerations that enhance SM $\pi^{+}$decaying to $\mu \nu$.
} 


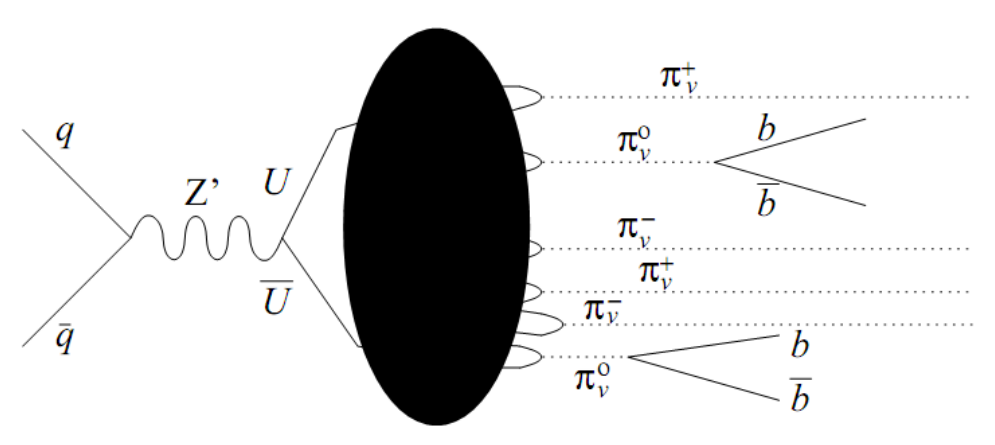

Figure 2.3: A possible v-particle production mechanism [17].

by [17],

$$
\Gamma_{\pi_{v} \rightarrow b \bar{b}} \sim 6 \times 10^{9} \sec ^{-1} \frac{f_{\pi_{v}}^{2} m_{\pi_{v}}^{5}}{(20 \mathrm{GeV})^{7}}\left(\frac{10 \mathrm{TeV}}{m_{Z^{\prime}} / g^{\prime}}\right)^{4}
$$

where $g^{\prime}$ is the coupling constant between the $Z^{\prime}$ and $Q$, analogous to the QCD coupling constant, $g$ and $f_{\pi_{v}}$ is the v-pion decay constant, analogous to the usual pion decay constant. Reference [18] suggests that for certain simple models, lifetimes of order $100 \mathrm{ps}(c \tau=3.8 \mathrm{~cm})$ may be possible for $20 \mathrm{GeV}$-pions decaying to heavy flavor. The parameters $m_{Z^{\prime}} / g^{\prime}$ and $f_{\pi_{v}}$ are unknown and may vary widely. As a consequence the v-pion may decay promptly or travel macroscopic distances. The most promising scenario, and the one considered here, is one that produces a long lived state corresponding to a light v-particle mass.

One possible mechanism for producing v-particles could occur through a $Z^{\prime}$ via $q \bar{q} \rightarrow Z^{\prime} \rightarrow U \bar{U}$ (as shown in figure 2.3). The precise details of the decay mechanism vary depending on the proposed model, however in each of them, the v-quarks hadronize due to confinement producing v-particles that are possibly long lived.

Another possible production mechanism and one which is of particular importance to this analysis is the possibility of a Higgs mixing with $\phi$ (as shown in figure 2.4), a 


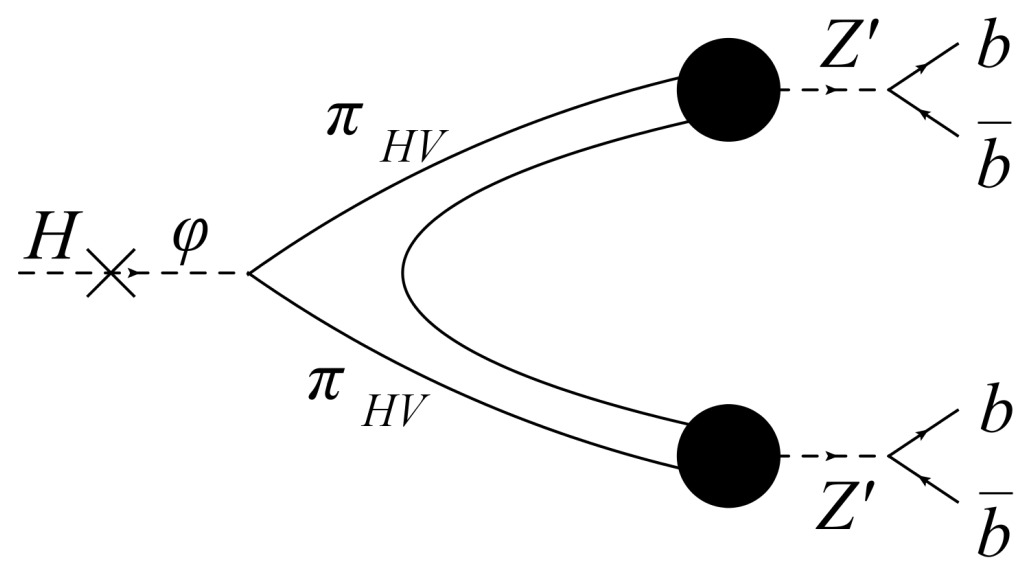

Figure 2.4: A possible Higgs decay through mixing [18].

HV Higgs boson that, in analogy to the SM, gives mass to v-particles. The $\phi$ could decay to v-hadrons which could either directly decay back to SM particles or mix through an intermediate, such as the $Z^{\prime}$, and then decay to SM particles. The SM Higgs could also decay directly to v-hadrons. The branching fraction of Higgs to v-hadrons could be substantial [18]. This particular model is used as a benchmark for the present analysis.

\subsubsection{Experimental Constraints on Hidden-Valley Models}

Experimental constraints on this hidden-valley model are minimal. From LEP1 there is an upper bound on the $\mathrm{Z}$ branching fraction of $10^{-7}$ for the process $Z \rightarrow U \bar{U}, C \bar{C}$ via $Z-Z^{\prime}$ mixing [19]. Cosmological constraints only require the existence of at least one light v-hadron with a lifetime $\ll 1$ second in order to preserve big-bang nucleosynthesis [17]. Other v-hadrons could be longer lived or even stable. Therefore, the only considerations limiting the reach of this analysis are experimental ones: the Tevatron is only capable of producing relatively light SM Higgs bosons and the size of 
the tracking portion of the $\mathrm{D} \varnothing$ detector determines the maximum observable v-hadron decay length ${ }^{2}$. The referenced hidden-valley models make no specific v-hadron mass or lifetime predictions and there is no HV model which is clearly more likely, however the simplest models which produce long-lived particles predict $m_{H V}$ in the range $20 \sim 40 \mathrm{GeV}$ (where the subscript HV is used to indicate any, generic v-particle).

The Higgs boson could serve as a window to a new sector of physics which would otherwise not interact with the SM. Given the dearth of experimental constraints on highly displaced vertices that result from HV models, it would not be surprising if the Higgs boson had already been produced and its decay had been entirely overlooked.

\subsection{Overview of the Analysis}

The signal for this analysis consists of at least two neutral particles, each one decaying to two b-jets after traveling at least $1.6 \mathrm{~cm}$, as illustrated in figure 2.5. This signature is completely generic and, although a specific model is considered, the results are applicable to a wide range of theoretically motivated hidden-sectors which produce long-lived states. The particular model used as a benchmark for this search is outlined in section 2.3.1 in which the Higgs boson mixes with a HV Higgs boson which decays to v-particles that are HV versions of the pion, or v-pions (see fig. 2.4). The vpions can then decay to a highly displaced $b \bar{b}$ pair. Although other analyses have looked for signatures that could be interpreted as having come from a hidden-sector, no other search for long lived v-particles with two highly displaced vertices has ever been undertaken and this analysis is completely novel.

\footnotetext{
${ }^{2}$ Our analysis method requires events that are fully contained. A different analysis using the muon system and missing energy could look for v-particles with a longer decay length. Such an analysis was proposed but will be left for future studies.
} 
Since multijet events comprise an enormous, irreducible background, it is necessary to search for events containing highly displaced vertices. Secondary-vertices (SV) are reconstructed by the tracking portion of the detector, which effectively limits the analysis to v-hadron decays occurring within the tracker volume, corresponding to a maximum radius of $50 \mathrm{~cm}$. In practice the maximum allowed radius is tightened; this is a fiducial volume cut because tracks are needed to reconstruct SVs. Additionally, previous studies have only verified MC to data vertex reconstruction agreement for radii less than $20 \mathrm{~cm} \mathrm{[12].} \mathrm{We} \mathrm{also} \mathrm{exclude} \mathrm{radii} \mathrm{less} \mathrm{than} 1.6 \mathrm{~cm}$ since this decay length is dominated by b-hadrons which are directly produced and whose decays are difficult to differentiate from signal.

Signal events are modeled with PYTHIA and a full detector simulation, as described in section 5.2.3. Figure 2.6 is a D $\varnothing$ event display in which a SM Higgs is produced near the primary vertex and decays in the silicon microstrip tracker via Higgs $\rightarrow$ $U \bar{U} \rightarrow \pi_{v}(\rightarrow b b) \pi_{v}(\rightarrow b b)$ where $\pi_{v}$ stands for any kinematically allowed v-particle.

Sources of highly displaced vertices other than signal, constituting the background, include the following:

- Charged pions and kaons are long lived and may decay in-flight producing tracks via $K_{S}^{0} \rightarrow \pi^{+} \pi^{-}, K^{ \pm} \rightarrow \mu^{ \pm} \nu_{\mu}$ and $\pi^{ \pm} \rightarrow \mu^{ \pm} \nu_{\mu}$, for example.

- Particles such as pions produced in the $p \bar{p}$ collision may inelastically scatter off the nuclei of detector material.

- Photons may produce $e^{+} e^{-}$pairs in the presence of a nucleus via, $\gamma+(N, A) \rightarrow$ $e^{+} e^{-}+(N, A)$.

- Multiple tracks coming from different decays could randomly coincide and be 


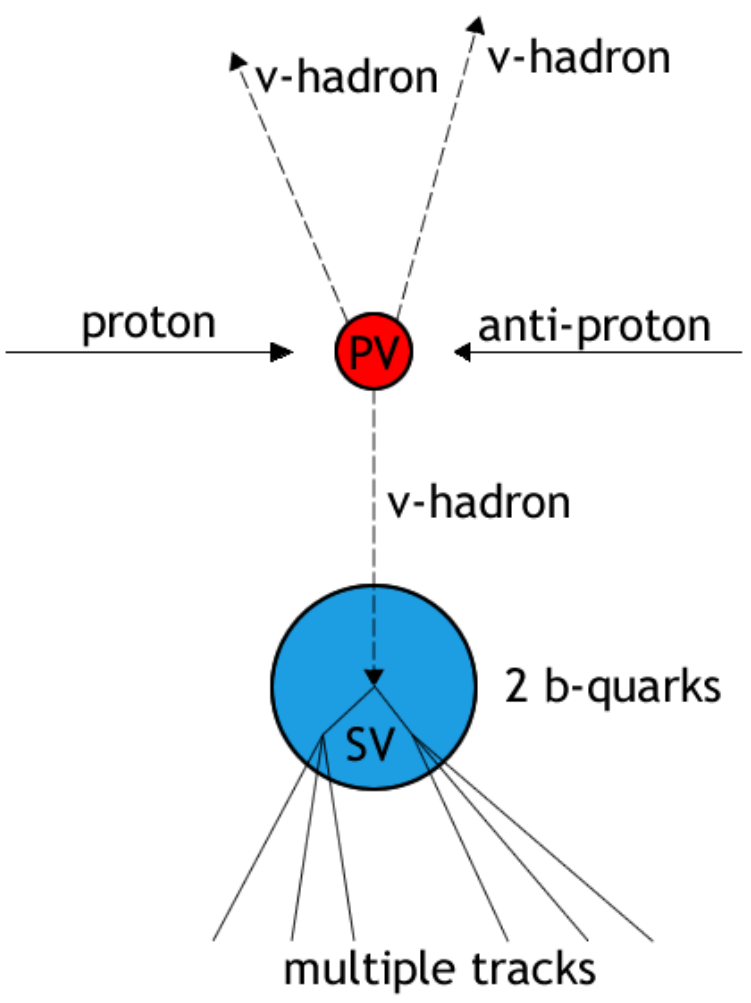

Figure 2.5: In this event, three v-hadrons are produced at the primary vertex (red circle), one of which decays in the tracker (blue circle). The secondary-vertex consists of two b-quark jets with their subsequent decays indicated by multiple tracks. 


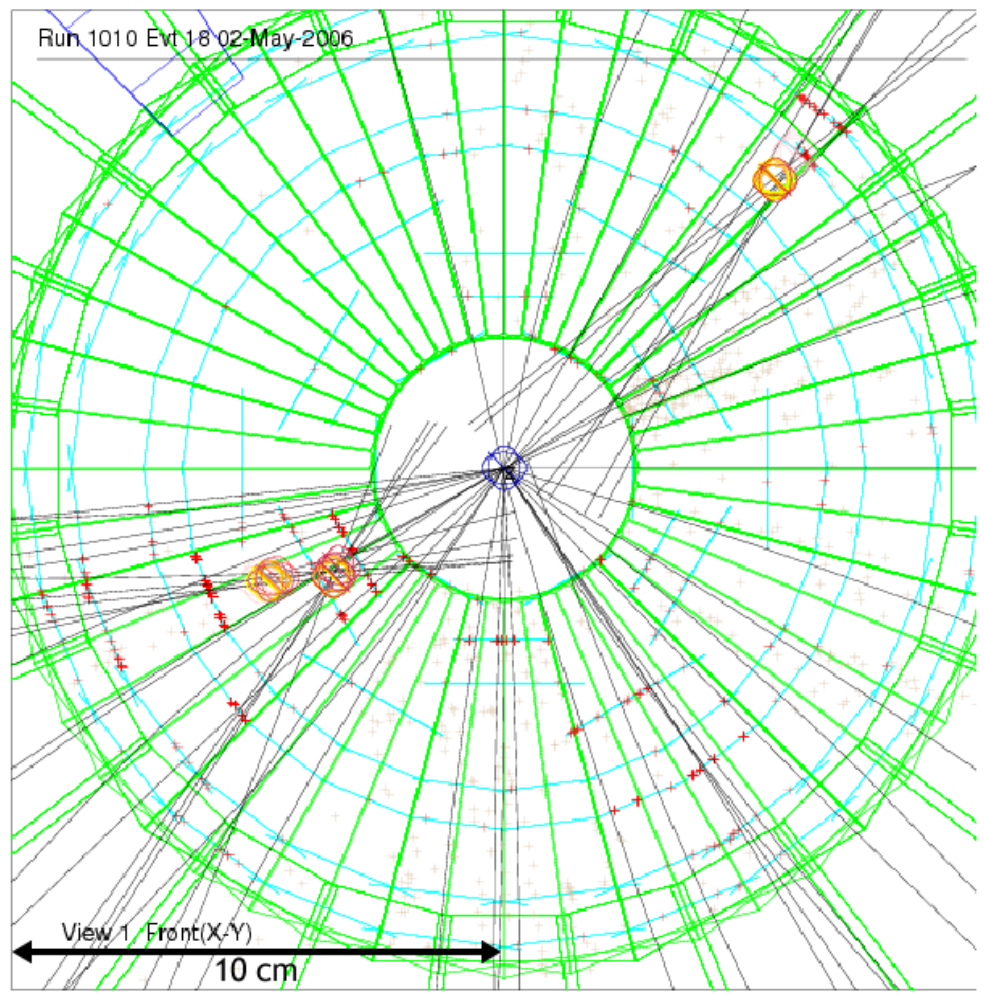

Figure 2.6: A MC simulated Higgs decay in the X-Y plane. The dark-blue primary vertex is in the center within the beam pipe which is at a radius of $1.6 \mathrm{~cm}$. There are three secondary vertices represented by the red-orange circles. Blue lines represent the material of the SMT ladders. 


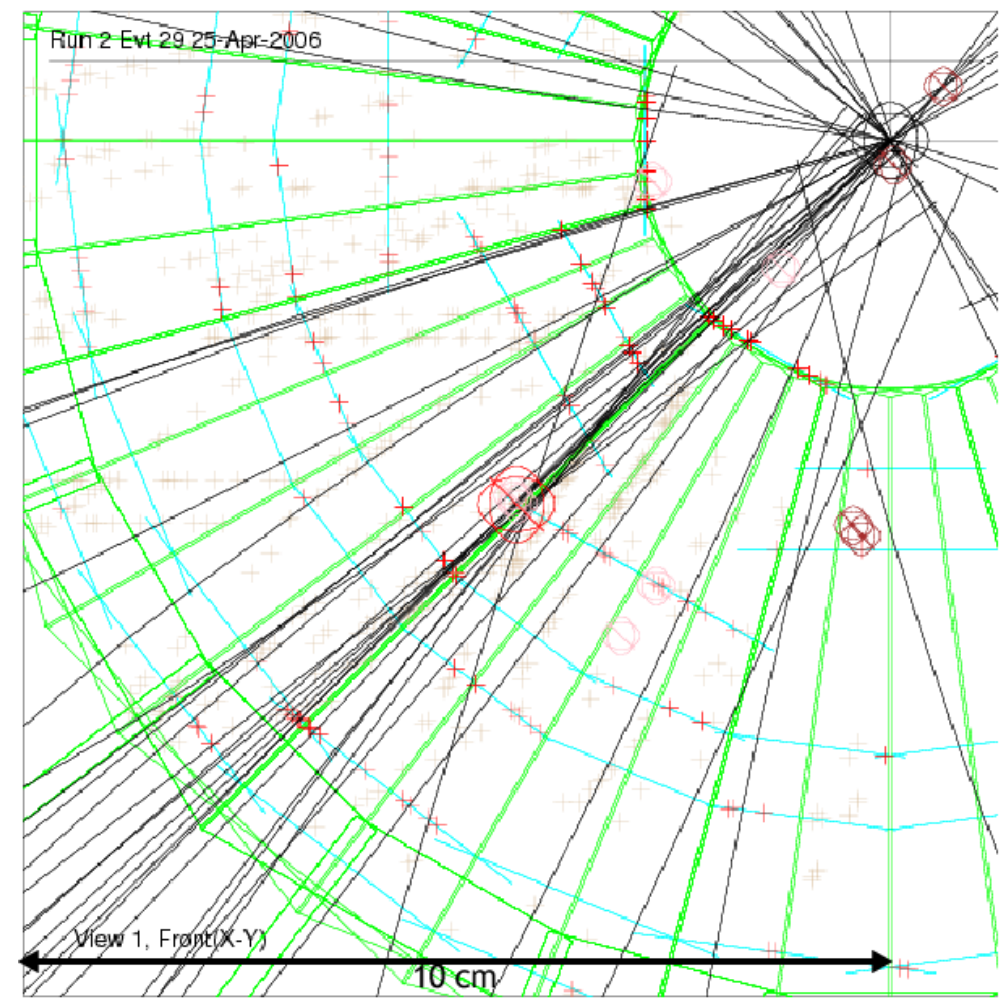

Figure 2.7: A MC simulated background event resulting from a material interaction. Six tracks are reconstructed to produce the secondary vertex which is located in a ladder of the SMT.

misreconstructed as having come from a common vertex.

- Problems with pattern recognition could misreconstruct a vertex from a group of tracks.

We model these processes with Monte-Carlo programs as discussed in section 5.2.2 and the methods of reconstruction are discussed in section 4 . Figure 2.7 is an example of a background event resulting from an interaction with the material of the SMT.

There are a few handles, as described in section 6.1, that can be used to distinguish background-like events from signal-like events. In section 6.2 we apply a loose set of cuts as a pre-selection, after which we normalize the background model to the data 
and apply MC corrections in section 6.3. Then in section 6.4 we make the final cuts which maximize the signal significance, defined as significance $=\frac{\text { signal }}{\sqrt{\text { signal+background }}}$. Finally, in section 6.6 we use the modified-frequentist approach to set a cross-section limit and discuss the effect of systematic uncertainties. The results of this analysis are the first constraints which have ever placed on a signature of this type. 


\section{Chapter 3}

\section{Experimental Apparatus}

This chapter describes how to produce high-energy proton-antiproton collisions and how to detect the particles that are created in such collisions. The data used in this analysis was collected during Run II of the Tevatron which began in 2001 and continues. Run II is a major upgrade from Run I which lasted from 1992-1995. The $\mathrm{D} \varnothing$ detector was also upgraded between runs. The upgraded D $\varnothing$ detector includes a new triggering system, a central solenoidal magnetic field and high-resolution track and vertex detectors.

\subsection{Units and Coordinates}

\subsubsection{Luminosity}

Luminosity is a measure of the intensity of a beam of particles and is determined by the density of particles in the beam. Instantaneous luminosity, $\mathcal{L}$ is the number of 
particles per unit area, per unit time and is related to the event rate by the expression,

$$
\text { Rate }=\frac{d N}{d t}=\sigma \times \mathcal{L}
$$

where $\sigma$ is the cross-section for a physics process. The cross-section is fixed for a given physical process and center-of-mass energy. The integrated luminosity is, $\int \mathcal{L} d t=N / \sigma$ where $\mathrm{N}$ is the total number of events. Cross-sections are measured in barns, which is $10^{-24} \mathrm{~cm}^{2}$. Although typical cross-sections are usually very small and measured in pico-barns $(\mathrm{pb})$ or $10^{-36} \mathrm{~cm}^{-2}$.

\subsubsection{Geometry}

Particle interactions are described in standard spherical coordinates centered at the interaction point: $\mathrm{r}, \phi$ (azimuthal), and $\theta$ as shown in figure 3.1. The $\mathrm{z}$ direction $(r=0)$ is defined along the beam-line. It is often convenient to use pseudo-rapidity instead of $\theta$ which is given by,

$$
\eta=-\ln \left(\tan \frac{\theta}{2}\right)
$$

Pseudo-rapidity is an approximation of rapidity in the massless limit which is given by,

$$
y=\tanh ^{-1} \frac{p_{Z}}{E}=\frac{1}{2} \ln \left(\frac{E+p_{Z}}{E-p_{Z}}\right)
$$

Under a boost in the z-direction to a frame with velocity $\beta, y \rightarrow y-\tanh ^{-1} \beta$. Hence, the shape of the rapidity distribution, $d N / d y$, is relativistically invariant or alternatively, the number of particles resulting from collisions is constant as a function of $y$ and (approximately) of $\eta$. 


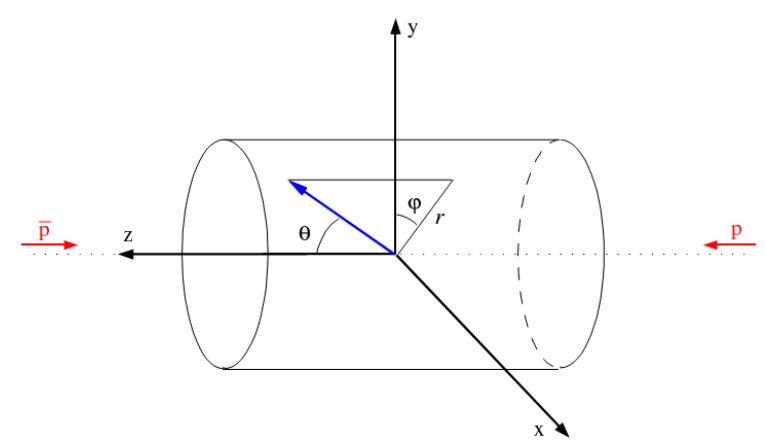

Figure 3.1: The $\mathrm{D} \varnothing$ coordinate system [20].

Solid angles are measured in terms of $\Delta R=\sqrt{\Delta \phi^{2}+\Delta \eta^{2}}$ which is also approximately invariant under boosts in the z direction.

\subsection{Physics Objects}

There are several physical objects that are special to high energy physics or collider experiments. The first of these is the jet. When quarks or gluons (which carry color) are produced in high energy collisions, they are boosted into different directions, however, color confinement demands that only color singlets can exist at large distances. The energy density surrounding a single quark increases until new quarks or antiquarks are spontaneously created from the vacuum in a process called hadronization. This process continues and more quark-antiquark pairs are produced resulting in a narrow cone of hadrons, known as a jet which is the object that is measured by the detector rather than the single quark, which hadronizes much too quickly to be directly observable. The jet will retain the original flavor of the first quark that gave rise to it so, for example, if a b-quark is created, the jet will have a net "b-ness" and it is possible to tag the jet as a b-jet. 
In a particle collision, a significant amount of energy may be carried away by particles that travel along the beam pipe. Collider detectors are, by necessity, not instrumented in the beam pipe and, consequently, the energy carried along the beam pipe will be unknown. As a solution to this problem, detectors measure the energy and momentum that is transverse to the beam: $E_{T}$ and $p_{T}$ (respectively). The calorimeter is segmented into cells and the transverse energy measured by the cell is given by, $E_{T}=E \cdot \sin \theta$ where $E$ is the energy measured by the cell and $\theta$ is the angle between the beamline ( $\mathrm{z}$ direction) and a ray pointing from the interaction point to the calorimeter cell. Because the incoming transverse momentum is zero missing transverse energy can be defined as, $M E_{T} \equiv E_{T}^{m i s s}=-\Sigma p_{T}$. In practice, this quantity is computed by adding up vectorially the transverse energies in all the calorimeter cells and subtracting any muon momenta, given by, $M E_{T}=-\left[\Sigma E_{T}\right]-p_{T}(l)$. If there is non-zero $M E_{T}$ it indicates energy that has escaped, usually due to non-interacting particles such as neutrinos.

Chapter 4 discusses the method of reconstructing physics objects from the measurements made by the elements of the detector.

\subsection{The Tevatron and Accelerator Chain}

The Fermilab Tevatron Collider $[21,22,23]$ is a $1 \mathrm{~km}$ radius superconducting synchrotron which is able to accelerate and store $980 \mathrm{GeV}$ beams of counter-rotating protons and antiprotons. The Tevatron collides the beams at two interaction regions which have a 3D Gaussian distribution with $\sigma \approx 25 \mathrm{~cm}$ in the $\mathrm{z}$ direction and $\sigma \approx 30$ $\mu m$ in the $\mathrm{r}$ direction. The protons and antiprotons are grouped into bunches of 36 


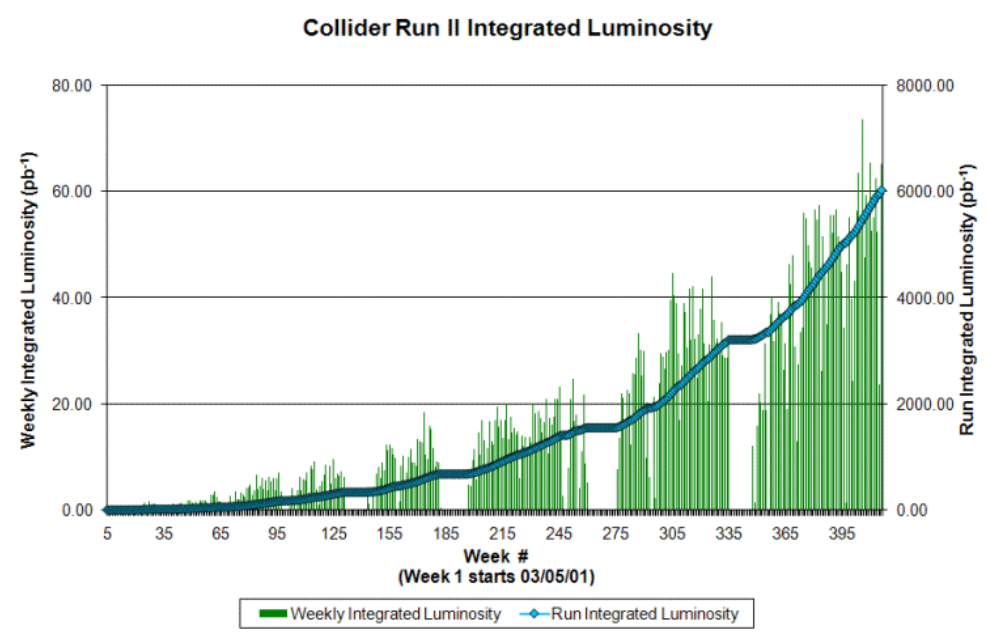

Figure 3.2: Integrated luminosity delivered by the Tevatron collider.

bunches per beam, corresponding to a bunch spacing of 396 ns. There are approximately $10^{13}$ protons and about a factor of 4 fewer antiprotons per bunch. Current peak instantaneous luminosities are in excess of $3.0 \times 10^{32} \mathrm{~cm}^{-2} \mathrm{~s}^{-1}$ and figure 3.2 shows the weekly and total integrated luminosities delivered from the beginning of Run II in March of 2001. Protons and antiprotons go through five unique stages of acceleration before they can be brought to their final energy of $980 \mathrm{GeV}$ in the Tevatron. What follows is a brief discussion of the various accelerator stages. Refer to figure 3.3 for a schematic of the accelerators at Fermilab.

\subsubsection{The Fermilab Accelerators}

Proton production begins with the Cockroft-Walton pre-accelerator in which hydrogen gas is ionized to create negative ions. Negative hydrogen ions come from a magnetron surface-plasma source [22]. The $H^{-}$ions are brought to $750 \mathrm{KeV}$, roughly 30 times the energy of the electron beam in a CRT, at which point they are injected 


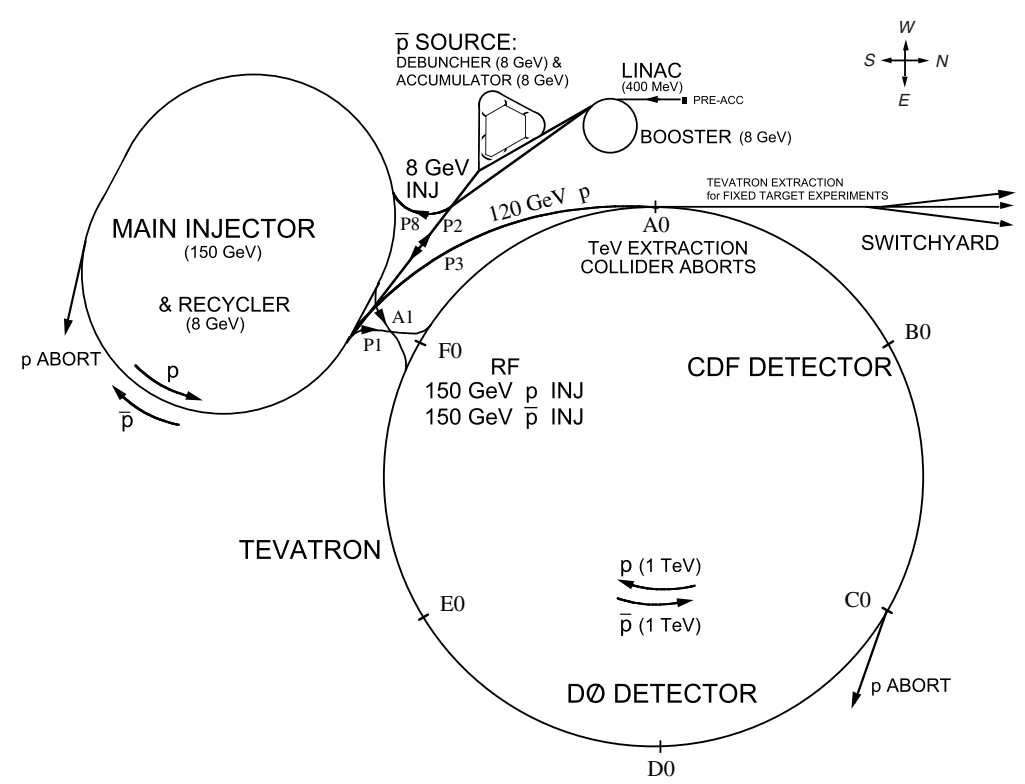

Figure 3.3: Fermilab's accelerator chain [23].

into a linear accelerator (LINAC). The LINAC is about 500 feet long and consists of RF cavities which produce oscillating electric fields. The LINAC accelerates the $H^{-}$ions to $400 \mathrm{MeV}$ before passing them through a carbon foil which strips away the electrons leaving the positively charged protons. The third stage of acceleration is the Booster which is a circular synchrotron of radius 151 meters. The booster groups the protons into bunches and brings them to an energy of $8 \mathrm{GeV}$ in $33 \mu s$ at which point the bunches are injected in the Main Injector. The Main Injector is a proton synchrotron serving 4 distinct purposes: 1) It accelerates protons from $8 \mathrm{GeV}$ to $150 \mathrm{GeV}$. 2) It produces $120 \mathrm{GeV}$ protons which are used for antiproton production 3) It receives antiprotons from the Debuncher and increases their energy to $150 \mathrm{GeV}$ and 4) It injects protons and antiprotons into the Tevatron. Antiprotons are produced when the Main Injector collides $120 \mathrm{GeV}$ protons onto a nickel target and focused by a lithium lens after which $8 \mathrm{GeV}$ antiprotons are selected by a dipole 
magnet. The Debuncher removes the bunch structure of the antiprotons and removes their momentum spread through stochastic cooling. From there, the antiprotons are stored in the Accumulator and Recycler until a sufficient number are collected to produce the required 36 bunches. The Main Injector tunnel also houses the antiproton recycler which stores antiprotons when the Accumulator is full and implements electron cooling to improve the quality and luminosity of the beam. At this point, 36 bunches of $150 \mathrm{GeV}$ protons are transferred to the Tevatron. Then 4 bunches at a time of antiprotons are transferred to the Main Injector, accelerated to $150 \mathrm{GeV}$ and then transferred to the Tevatron. Once 36 bunches of protons and antiprotons are present, the Tevatron accelerates them to $980 \mathrm{GeV}$ in a single process. The beams are "scraped" and brought into focus at the collision points. Beam scraping uses collimators to remove halos which occur when protons or antiprotons posses a momentum different from that of the main bunch. Finally, a store is declared and collisions continue until the quality of the beams have degraded to the point where they are no longer in focus or of sufficient luminosity. Usually after about 20 hours the store ends and the beams are dumped.

\subsubsection{Proton-Antiproton Collisions}

Protons are composite particles and consequently, collisions occur between the quark and gluon constituents, or partons. Most collisions result in a small momentum transfer, or soft-scatter. The more interesting case occurs in a hard-scatter, when the partons directly interact with one another and there is a large amount of energy made available in the collision. The hard-scattering interaction may result in the production of intermediate resonances, such as the $Z$ or Higgs boson. These resonances are 
typically very short lived, much too short to be directly observed by the detector elements, although their decay products are semi-stable and can be measured.

Two classes of particles, hadrons and leptons, are created following the decay of a heavy particle. The momenta of charged particles are measured in the tracking portion of the detector which is immersed in a solenoidal magnetic field. Electrons and photons produce showers in the calorimeter, where their energy is measured. Hadrons are more penetrating and deposit their energy deeper in the calorimeter. Muons generally are not absorbed in the calorimeter and consequently escape to the muon chambers where their momenta are measured using a toroidal magnetic field. Neutrinos escape the detector and can only be measured by requiring energy conservation.

\subsection{The DØ Detector}

Although the DØ Run II detector [24] is described elsewhere, this section will briefly describe the main components that are important for this analysis. Figure 3.4 shows an elevation view of the detector. The innermost portion of the detector is the beryllium beam pipe which is $2.37 \mathrm{~m}$ long, has a diameter of $38.1 \mathrm{~mm}$ and a wall thickness of $0.508 \mathrm{~mm}$. Within the beam pipe, $p \bar{p}$ collisions occur in vacuum.

\subsubsection{The Inner Detectors}

The inner detectors perform tracking and luminosity measurement. Excellent tracking is essential for most physics studies at $\mathrm{D} \varnothing$ and the present analysis depends heavily on tracking. There are two separate tracking detectors which are described 


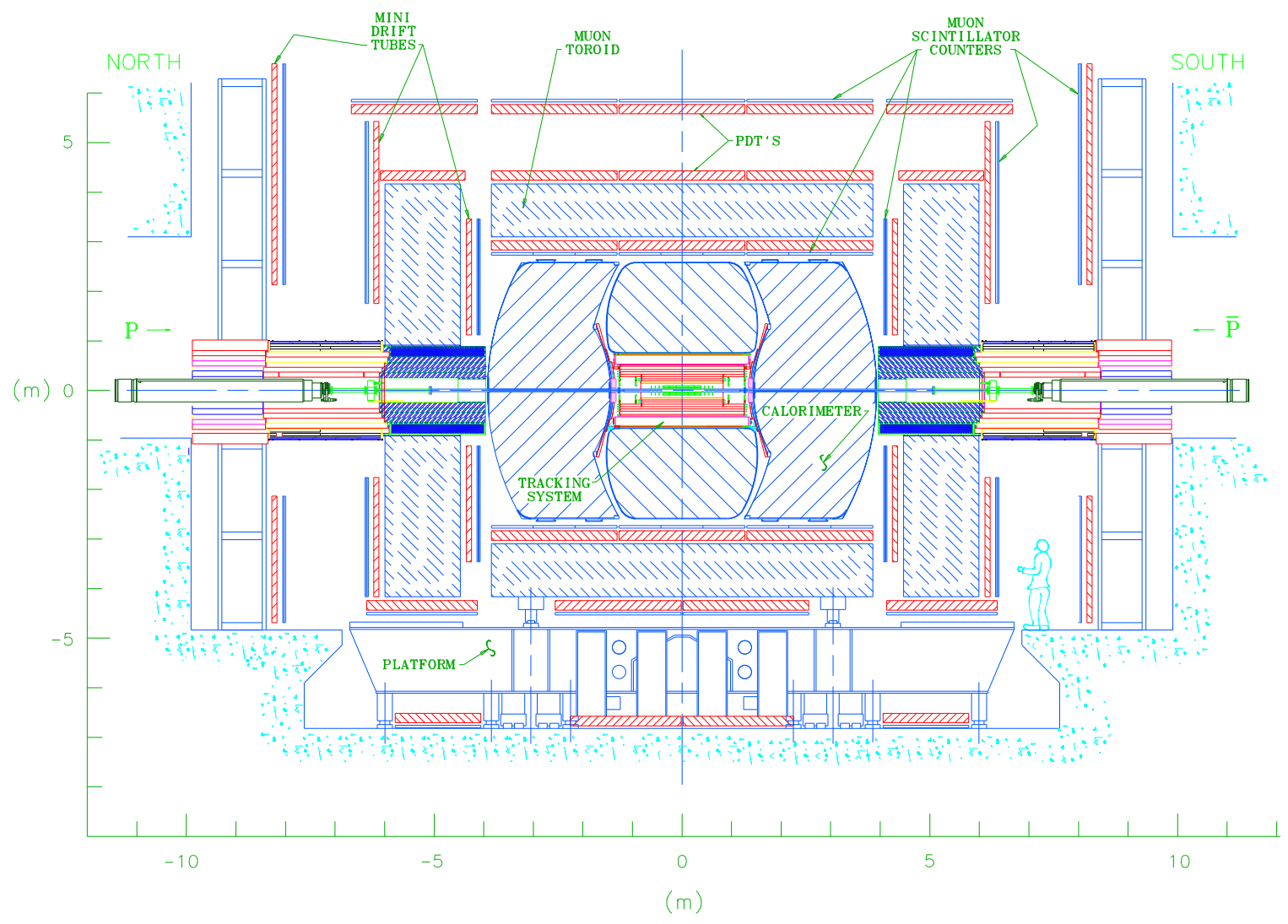

Figure 3.4: The upgraded D $\varnothing$ detector for Run II. Protons enter from the left while antiprotons enter from the right. Collisions occur near the center of the detector. The innermost portion of the detector consists of the tracker which is contained within a solenoidal magnet. The calorimeter is in the central portion and the muon system, which contains an iron toroid magnet, comprises the outermost portion. 
in the following sections and together they can determine the location of the primary interaction point (primary-vertex) with a resolution of about $35 \mu \mathrm{m}$ and they can measure the impact parameter of b-quark jets with a resolution of about $15 \mu \mathrm{m}$.

\subsubsection{The Luminosity Monitor}

The luminosity detector consists of plastic scintillators mounted in front of the end-cap calorimeters and covers the $\eta$ range, $2.7<\eta<4.4$ (ie. very close to the beam pipe). In figure 3.6 the luminosity monitor is labeled as "level 0". At D $\varnothing$ the luminosity is measured by counting the rate of inelastic $p \bar{p}$ collisions. Inelastic collisions produce charged fragments which interact with the plastic scintillator to produce light pulses which are recorded by photo-multiplier tubes. The probability of $n$ interactions per beam crossing is given by a Poisson distribution of mean $\mu$,

$$
P(n)=\frac{\mu^{n}}{n !} e^{-\mu}
$$

The probability of at least one interaction is then given by,

$$
P(n>0)=1-e^{-\mu}
$$

where $\mu=\mathcal{L} \times \sigma_{\text {eff }} /$ crossing rate which implies that the luminosity is,

$$
\mathcal{L}=-\frac{\text { crossing rate }}{\sigma_{\text {eff }}} \ln (1-P(n>0))
$$

The effective cross-section, $\sigma_{\text {eff }}$ consists of three parts: 1) the cross-sections for for hard-core, single-diffractive and double-diffractive processes, 2) the acceptance for 
each process and 3) the detection efficiency of the luminosity monitor counters. The cross-section for each process is known from previous experiments. In this way, the luminosity is measured by counting the number of beam crossings in which there is no inelastic collision [25].

\subsubsection{The Silicon Microstrip Tracker}

The silicon microstrip tracker (SMT) measures the location of charged particle tracks with a resolution of about $10 \sim 35 \mu \mathrm{m}$ depending on location. When a charged particle traverses a p-n junction in silicon it ionizes the material, creating electronhole pairs. By applying a bias voltage, the pairs drift apart until they reach conducting strips which are implanted in the silicon. Each silicon wafer is about $300 \mu m$ thick while the conducting strips are about $50 \mu \mathrm{m}$ wide. Charge from these strips are collected by a capacitor and then read-out and digitized. The microstrips used in the detector are single-sided or double-sided. Double sided microstrips allow position to be localized in more than one direction by rotating the strips relative to each other, either by $2^{\circ}$ or $90^{\circ}$.

The SMT is located immediately outside of the beam pipe and occupies the radial distance to about $10 \mathrm{~cm}$ and provides coverage for $|\eta| \leq 3.0$. Along with the Central Fiber Tracker $(\mathrm{CFT})$ it is located within a $2.0 \mathrm{~T}$ axial magnetic field provided by a solenoid. The magnetic field allows for precise momentum resolution. The SMT is designed to cover the entire interaction region, which is distributed along a distance of about $25 \mathrm{~cm}$ along the beam line. The SMT requires that tracks from charged particles traverse the silicon strips roughly perpendicularly which necessitates that some strips are parallel to the beam (barrels) and some are perpendicular to the beam (disks). 


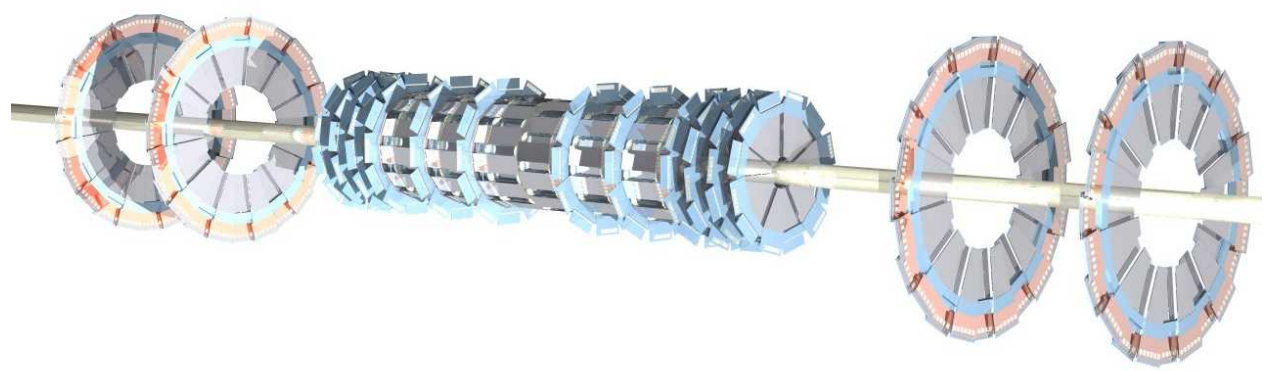

Figure 3.5: The silicon microstrip tracker geometry. Six barrels cover the central region (about $|\eta| \leq 1.1)$ while the F-disks and H-disks cover the high $\eta$ region $(|\eta| \leq$ 3.0) [27].

There are six $12 \mathrm{~cm}$ long barrels comprising four detector layers interspersed with 12 disks ("F-disks) for forward tracking. In the very far-forward regions there are larger disks ("H-disks") which cover the region $2<\eta<3$. The barrels have an inner radius of $2.7 \mathrm{~cm}$ and an outer radius of $9.4 \mathrm{~cm}$ while the $\mathrm{F}$-disks have an inner radius of $2.6 \mathrm{~cm}$ and an outer radius of $10 \mathrm{~cm}$. The larger H-disks have an inner radius of $9.6 \mathrm{~cm}$ and an outer radius of $23.6 \mathrm{~cm}$. Figure 3.5 shows the SMT geometry while figure 3.6 shows the location of the SMT relative to the rest of the DØ detector. After approximately $1 / 3$ of the data for this analysis was collected, an additional inner layer ("layer 0") was installed. Layer 0 fits inside of the existing SMT barrels at a radial distance of $1.6 \mathrm{~cm}$, just $6.8 \mathrm{~mm}$ outside of the beampipe [26]. This upgrade occurred between run $2 \mathrm{a}$ and run $2 \mathrm{~b}$ and its presence is accounted for in the analysis.

\subsubsection{The Central Fiber Tracker}

The second component of DØ's tracking system is the central fiber tracker (CFT) which is located within the solenoid, just outside of the SMT (refer to figure 3.6). The CFT extends from from a radial distance of about $20 \mathrm{~cm}$ to $52 \mathrm{~cm}$ from the 
beam pipe and can measure position with a resolution of about $100 \mu \mathrm{m}$ assuming the position of the fiber is well measured. The CFT consists of scintillating fibers of radius $835 \mu \mathrm{m}$ which are mounted on eight concentric cylinders. In order to allow space for the SMT's H-disks, the two inner cylinders are $1.66 \mathrm{~m}$ long while the six outer cylinders are $2.52 \mathrm{~m}$ long and provide coverage for $|\eta| \leq 1.7$. Each cylinder carries two doublet layers of fiber; one layer points in the radial direction while the other points at a stereo angle of $+3^{\circ}$ or $-3^{\circ}$. The orientation of the stereo layer alternates $(+)$ or $(-)$ for each successive cylinder.

The fibers are composed of an organic scintillating compound surrounded by two layers of cladding which provide total internal reflection. The fibers are wavelength shifting which prevents the scintillation light from being reabsorbed. The wavelength shifting dye absorbs the original $340 \mathrm{~nm}$ radiation and re-emits it at $530 \mathrm{~nm}$. The light is brought out to visible light photon counters (VLPCs) via clear waveguides of the same diameter as the individual fibers. Because only one end of the fiber is read-out, the other end is coated with reflective aluminum which reflects about $90 \%$ of the light back to the other end. The CFT is a very fast detector because of the rapid fluorescence which decays after only a few nanoseconds.

\subsubsection{The Calorimeter}

A sampling calorimeter lies outside of the central tracker and solenoid in a region of low magnetic field. The purpose of the calorimeter is to measure the energy of electromagnetically interacting particles and strongly interacting particles. The absorber material induces electromagnetic and hadronic showering while the resulting charged particles ionize the active material. The active material is in an applied electric field 


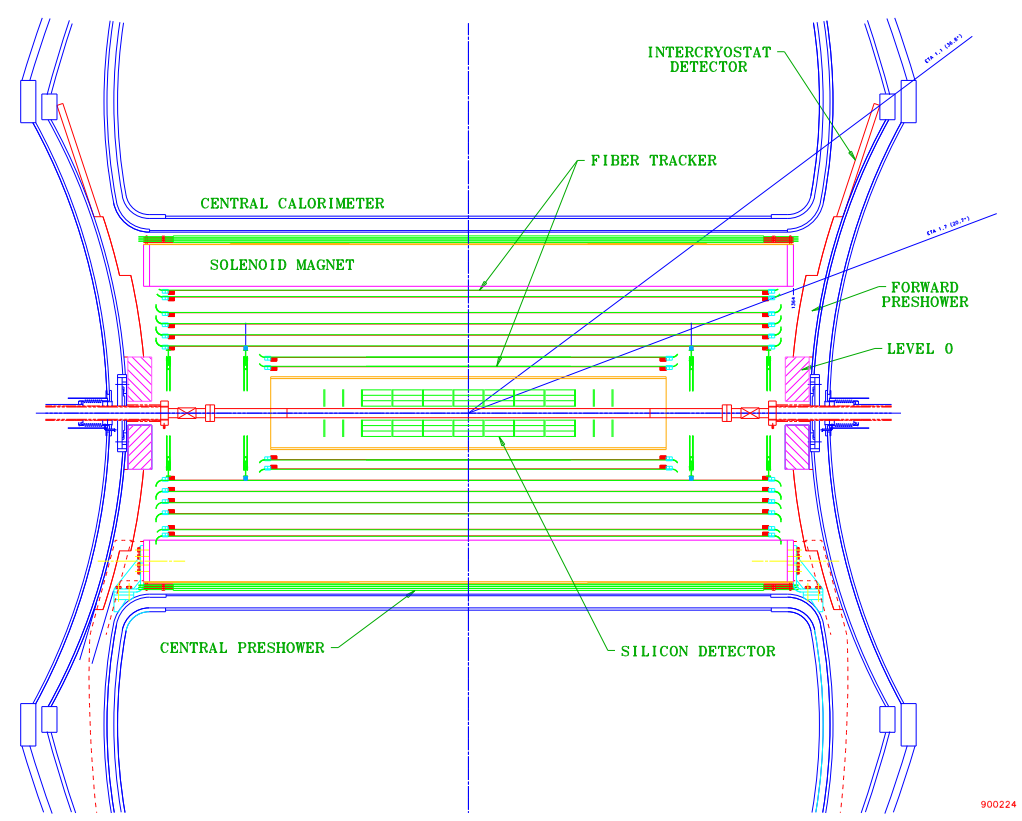

Figure 3.6: Cross-sectional view of the inner detectors in the x-y plane [24].

which separates the electrons and ions that result from ionization. The charge that is collected is then read-out and recorded as an electrical pulse. The amount of charge is proportional to the total energy of the ionizing particle.

There are three sub-detectors which make up the calorimeter: the central region (CC) which extends to $|\eta|$ of 1.1 and two end-cap regions (EC) which extend to $|\eta|$ of 4.2, as shown in figure 3.7. Each region is housed in a separate cryostat. The regions between the CC and EC are covered by a separate detector called the inter-cryostat detector so that the whole calorimeter is nearly hermetic. The calorimeter is further subdivided into three additional detector regions which are, in order of distance from the beamline: the electromagnetic region, the fine hadronic region and the coarse hadronic region. The electromagnetic portion represents 21 radiation lengths while the combined fine and coarse hadronic portions adds an additional 7 radiation lengths to the detector. The numerous radiation lengths are important to ensure that a 


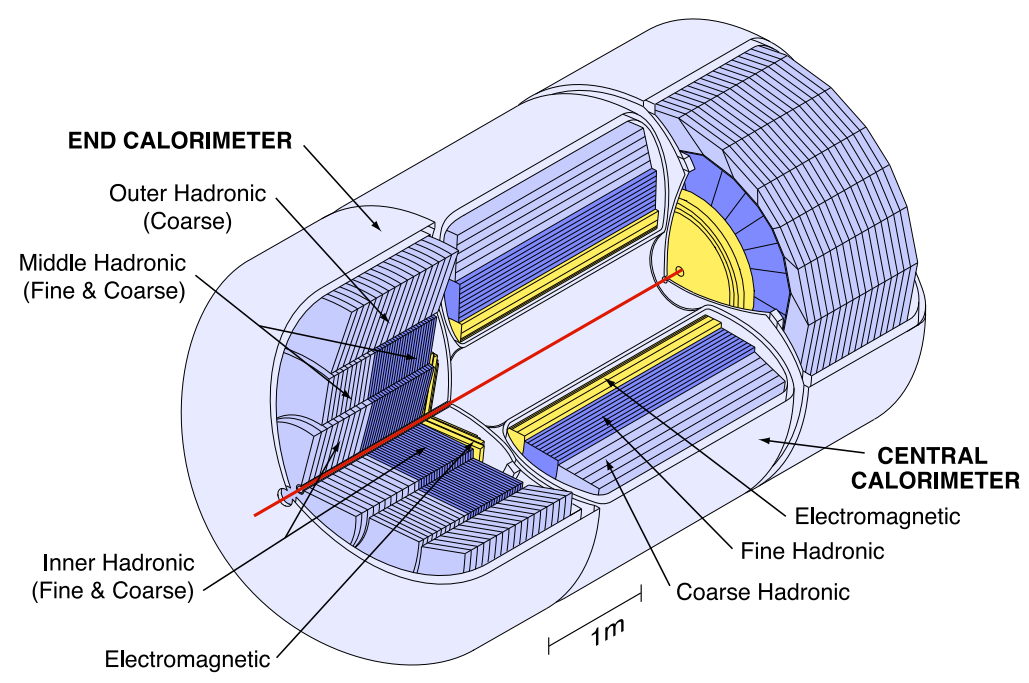

Figure 3.7: A 3D view of the calorimeter. The central and end-cap calorimeters are contained in separate cryostats [24].

particle deposits nearly all of its energy in the detector. The calorimeter is composed of read-out cells that form pseudo-projective towers with each tower subdivided in depth (refer to figure 3.8). The size of each tower in $\eta$ and $\phi$ is $0.1 \times 0.1$.

The absorber material is made from depleted uranium in the electromagnetic section, uranium-niobium alloy in the fine hadronic section and copper (in the CC) or stainless steel (in the coarse hadronic section). Both pions and electrons create the same ionization per unit length in uranium making it an ideal absorber material for the electromagnetic portion of the calorimeter. Between each layer is the read-out cell which contains liquid argon. Within each cell, a copper pad is held at high voltage to create an anode which collects the ionization. A typical calorimeter cell is shown in figure 3.9 . 


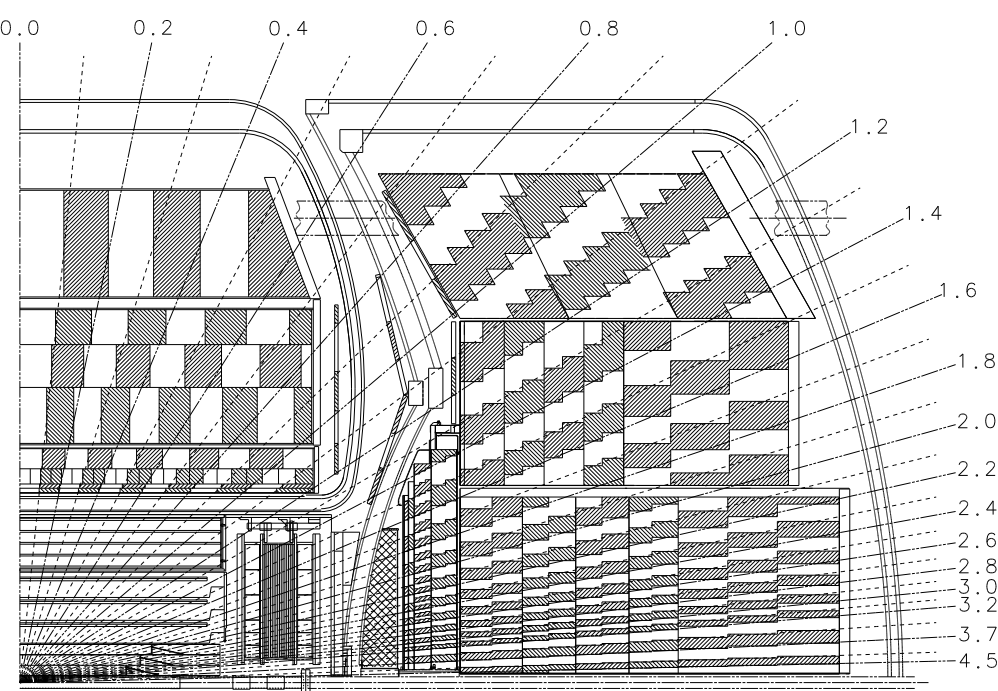

Figure 3.8: A side view of the calorimeter. The calorimeter is segmented into cells of size in $\eta$ and $\phi$. In the electromagnetic and fine hadronic portion the cell size is $\eta \times \phi=0.1 \times 0.1$ while in the course hadronic portion the cell size is $\eta \times \phi=0.2 \times 0.2$ [24].

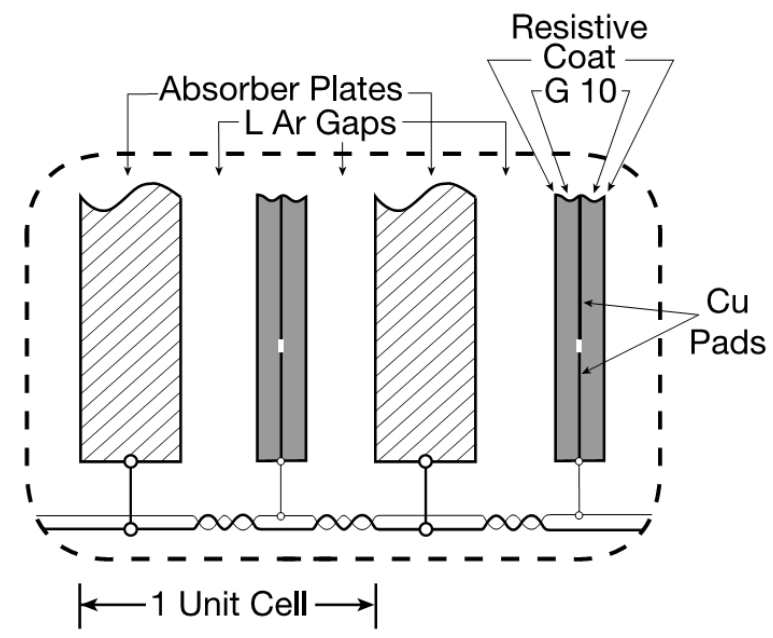

Figure 3.9: Schematic view of the liquid argon gap and signal board unit cell for the calorimeter [24]. 


\subsubsection{The Muon System}

Because most muons pass through all of the detector elements previously mentioned, the outermost portion of the detector is the muon system. The purpose of the muon systems is to measure the muon momentum. The muon system consists of a 2000 ton, 1.9 Tesla iron toroid magnet and three active detector layers that measure particle position. Muon momentum is determined by measuring the bending of the muon's path which results from its passage through the magnetic field. One position measurement is performed before the muon passes through the magnetic field (in the A layer) and two measurements (in the $\mathrm{B}$ and $\mathrm{C}$ layers) are performed after it passes through the magnetic field. The upgraded Run II detector added a magnetic field and tracker to the central region of the detector which also performs momentum measurement, usually more accurately. Nevertheless, the muon system is still necessary for muon identification and is more accurate for momentum measurements of muons with $|\eta|>1.6$. Each detection layer consists of fast scintillation counters for triggering and drift chambers for precise position measurement. Timing information from the scintillation counters allows for coincidence determination and, therefore, cosmic rays can be rejected. Muon identification is further assisted by combing information from the central tracker and the calorimeter where muons typically deposit about 3 $\mathrm{GeV}$ of energy.

A three dimensional layout of the muon system in relation to the rest of the detector is shown in figure 3.10 . 


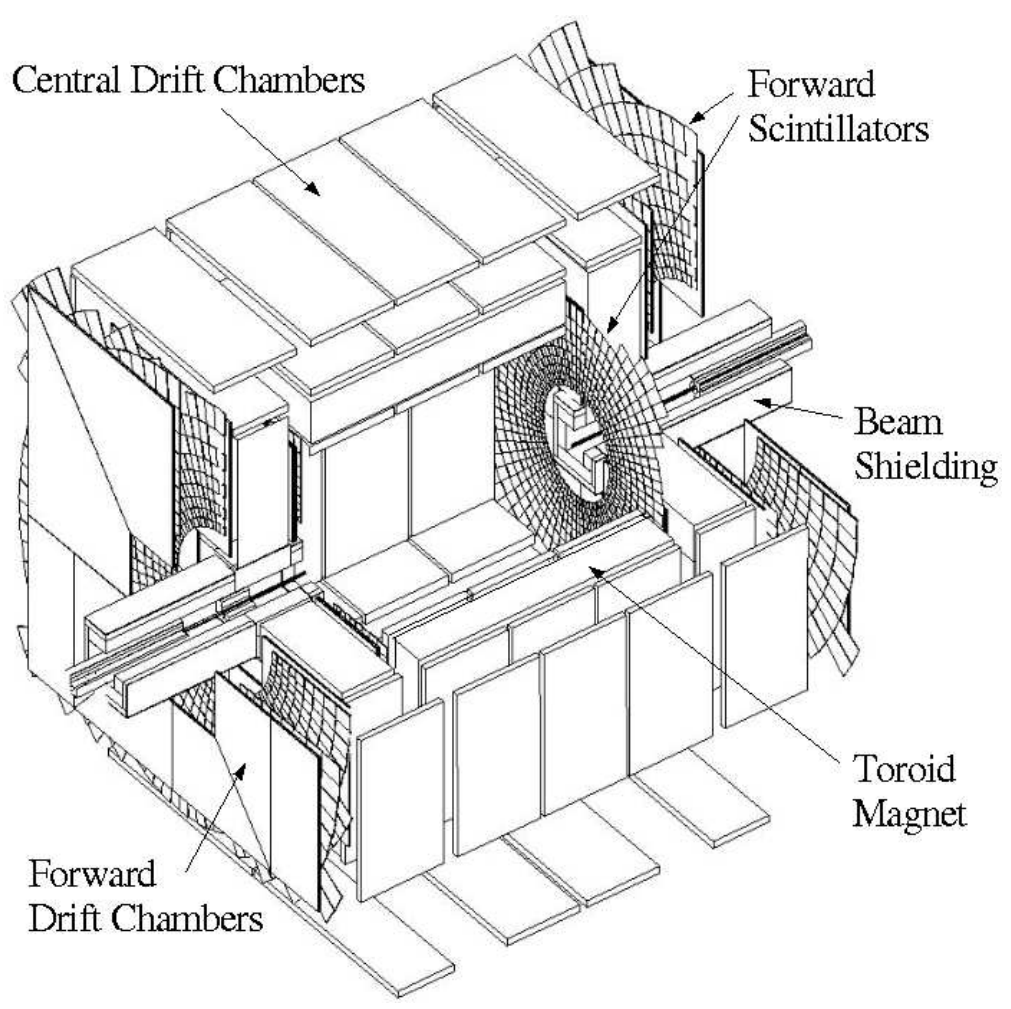

Figure 3.10: The DØ muon detector. The bottom of the detector (not shown) is not fully instrumented and contains holes for the support structure and wiring. The toroid manget sits between the A and B layers [28]. 


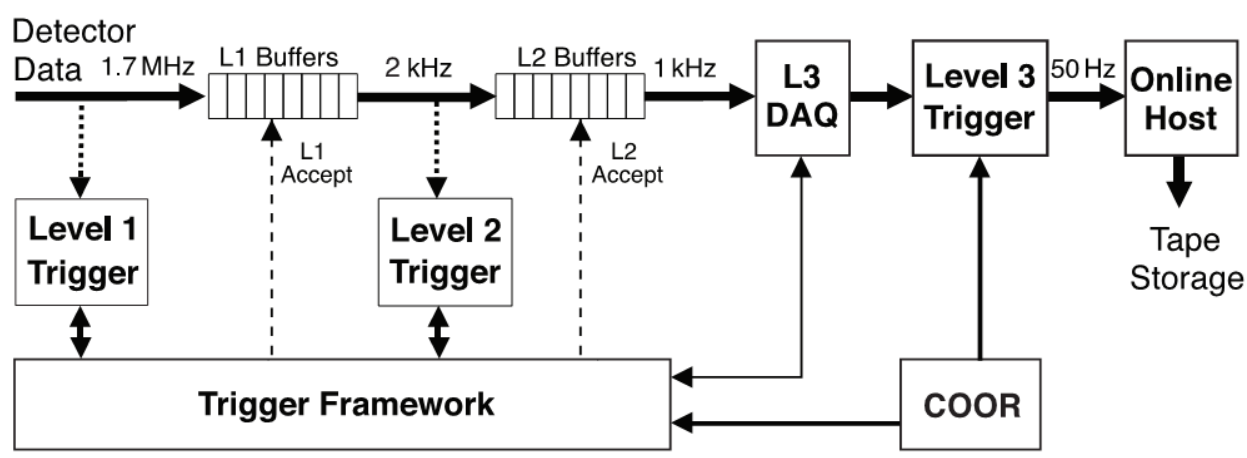

Figure 3.11: Overview of the D $\varnothing$ trigger and run control systems [24].

\subsubsection{Triggering and Data Acquisition}

The Tevatron produces collisions every 396 ns for a $1.7 \mathrm{MHz}$ event rate, yet hardware and offline resource limitations only allow for $\mathrm{D} \varnothing$ to record events at a rate of about $100 \mathrm{~Hz}$. A simple trigger system could simply throw away events to bring the rate down (known as prescaling) however, most collisions either do not result in a hard scatter or simply do not contain an "interesting" event. DØ implements a three level trigger system in order to maximize the usefullness of the $100 \mathrm{~Hz}$ of bandwidth that is available and only record event that contain potentially interesting physics. Each event must pass each successive trigger level to be considered by the next level. Each level also sees a smaller rate of events and has more detector information on which to base its decision. Figure 3.11 shows a block diagram of the trigger and data aquisition system. The following describes the major components of the trigger system used in this analysis.

The level one trigger (L1) takes as input simplified signals from four different detector subsystems: the calorimeter, the central fiber tracker, the muon system and the forward proton detector. The L1 trigger examines every event for interesting 
features and reduces the event rate to about $2 \mathrm{kHz}$. The trigger decision must arrive at the trigger framework in $3.5 \mu s$ or less. It is implemented in specially designed hardware and sends its trigger decision to the front end electronics and on to the next level of triggering, L2. The two L1 triggers used for this analysis are the calorimeter trigger (L1Cal) and the muon triger (L1Muon). The L1Cal sums calorimeter cells together and forms trigger towers which are cell groups of size $0.2 \times 0.2$ in magnet $\eta \times \phi$. The event is passed by the trigger if there are a certain number of towers with energy above a predetermined threshold. The L1Muo examines tracks in the muon system and passes events if a certain number of tracks exceed preset thresholds in transverse momentum.

In the second trigger stage (L2), the event rate is further reduced to about 1 $\mathrm{kHz}$. The trigger takes as input, the information from L1 and slightly more, though still simplified, information from the detector subsystems. L2 uses special hardware and embedded microprocessors associated with each detector subsystem. Because the input rate is less than L1, the L2 trigger can spend more time on each event allowing it to consider more sophisticated physics objects and correlations between them. For example, the L2 trigger implements a simple jet reconstruction algorithm by summing trigger towers around a candidate. This analysis only uses the L2 muon and jet systems.

The level three trigger (L3) is software based and implemented on a computer farm which reduces the event rate by a factor of 10. After a L2 trigger accept, the full data from the event under consideration is read-out from each detector subsystem and then processed on a single computer. If the event passes the L3 trigger, it is recorded to tape. The algorithms used at L3 are slightly simplified, though very similar to the 
full offline reconstruction. Approximately $100 \mathrm{~ms}$ are spent per event.

\section{Run 2b Trigger Upgrade}

Between runs $2 \mathrm{a}$ and $2 \mathrm{~b}$, major upgrades were made on several of the trigger subsystems: the L1 calorimeter (L1Cal), the L1 central track trigger (CTT), the L2 silicon track trigger (STT) and a new L1 system to match calorimeter energy clusters with tracks (Cal-TRK). This analysis is only affected by the upgrades performed on the L1Cal and the L1 CTT.

The L1Cal upgrade uses the same calorimeter trigger towers as in run $2 \mathrm{a}(0.2 \times 0.2$ in $\eta$ and $\phi$ ) but performs a local-maxima finding routine on groups of trigger towers instead of only considering single towers. The new algorithm moves a window grid across the calorimeter's $\eta, \phi$ space to find local maxima, which is known as the "sliding windows" algorithm. This has the advantage of finding true local-maxima (instead of single towers) and allows for accounting of differently shaped energy deposits. For example, tau leptons deposit energy in a tighter cone of energy than a hadronic jet. The new scheme also allows for isolation criteria; requiring that adjacent trigger towers not exceed a certain threshold.

The upgraded L1 CTT trigger improves the simplified CFT track finding algorithm that is implemented at L1. During run 2a the algorithm only considered pairs of neighboring fibers, referred to as a "doublet". In this scheme, a track must pass through two fibers mounted on a single cylinder. The run $2 \mathrm{~b}$ upgrade allows for particle tracks to pass through only a single layer of fibers per cylinder as shown in figure 3.12. 


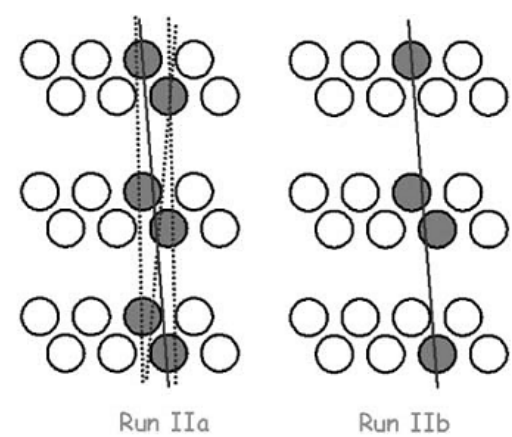

Figure 3.12: The doublet (left) and singlet (right) schemes for the CTT triggers. The circles represent fibers and the solid line represents the actual track trajectory [29]. 


\section{Chapter 4}

\section{Reconstruction and Calibration}

Reconstruction is the process of taking low level data collected by the D $\varnothing$ detector

and identifying it with physics objects. This chapter describes how small amounts of energy deposited in each detector element are used to reconstruct the full event. This analysis relies on four basic physics objects indicated by the headings of the following sections: Tracks (section 4.1) represent the path taken by particles as they traverse the detector and are measured when the particles deposit small amounts of energy in the SMT or CFT. When a particle decays or when multiple particles interact, their tracks intersect at a vertex (section 4.2). This analysis looks for long lived particles that decay to highly displaced vertices, therefore accurate measurements of the locations of the primary and secondary vertices are of utmost importance. Strongly interacting partons produce a cone of particles through hadronization and these are observed as jets in the calorimeter (section 4.3). Finally, muons penetrate most of the detector and are identified by their presence in the outer portion of the detector (section 4.4). Both jets and muons are used at the trigger level to maximize 
the probability of observing a signal-type event. As a final step, the response of the detector to a given amount of energy deposition must be calibrated in order to make real measurements of quantities like transverse energy or momentum.

The same reconstruction algorithms described in this chapter are applied to both data and Monte-Carlo simulated events.

\subsection{Tracking}

The purpose of the tracking system is to measure, with great accuracy, the location of particles and their momenta. Location can be determined by causing the particle to interact and deposit energy in pieces of the detector whose locations are known very precisely. The momentum of a particle in a magnetic field can be determined from the position measurements if the particle is charged in which case its path will be that of a helix. In a magnetic field, a charged particle's transverse momentum is given by

$$
p_{T}=\frac{e B}{\rho}
$$

where $\rho$ is the curvature of the particle's trajectory, $e$ is the particle's charge and $B$ is the external magnetic field.

\subsubsection{Hit Clustering}

The tracking system determines the path taken by charged particles by reconstructing a series of "hits" left behind in the SMT and CFT. Hits are points where energy is measured; either charge gathered by the microstrips in the SMT or light collected by the fibers in the CFT. If an adjacent SMT strip registers a hit, then they are 
combined in a process called track hit clustering. The center of the hit is given by the charge weighted average of the center of each silicon strip. It should be noted that the SMT is immersed in a magnetic field and as a consequence, the electrons and holes do not drift along the electric field lines but at an angle called the Lorentz angle which must be corrected for when calculating the center of the SMT hits. The Lorentz angle depends on the magnetic field strength, the electron or hole Hall-mobility, and the drift velocity in silicon. The measured values are $18^{\circ}$ for electrons and $4^{\circ}$ for holes [30]. The position of hits are reconstructed to within $10 \mu \mathrm{m}$ in the axial direction, 35 $\mu m$ in the $z$ direction for $90^{\circ}$ stereo and $450 \mu m$ in the $z$ direction for $2^{\circ}$ stereo [31].

Track hit clustering is also performed in the CFT when adjacent fibers register a hit. The center of the cluster is simply the average position of the fibers. Due to the $3^{\circ}$ relative angle between doublet-layers, the intersection of two overlapping fibers gives the $\mathrm{x}-\mathrm{y}$ coordinates. The resolution of the CFT is about $100 \mu \mathrm{m}$ in the axial direction and about $2 \mathrm{~cm}$ in the $z$ direction [28].

To measure the position of an individual particle, the location of the detector itself must first be known with respect to the other detector elements. The support structures of the CFT and the SMT are machined and placed with great care and their locations are optically surveyed, however to achieve the very high accuracy that is necessary, the location of the sub-detectors must be calibrated in situ by use of cosmic rays or particles from $p \bar{p}$ collisions.

\subsubsection{Track Finding}

At present, there are two distinct algorithms which are used to fit tracks to hits; histogram track finding (HTF) and the alternative algorithm (AA). Both methods are 
used to reconstruct all tracks and the results are combined at the end and smoothed using the Kalman algorithm.

\subsubsection{Histogram Track Finding}

The histogramming track finder (HTF) algorithm implemented at DØ employs a method originally invented in the 1950's for bubble chamber experiments [32]. The algorithm uses a Hough transform to map pairs of points to lines in parameter space [33]. The lines intersect at a point corresponding to the parameters of the trajectory in question. Given a hit at point $(\mathrm{x}, \mathrm{y})$ from a track originating at the origin and having curvature $\rho$ (from equation 4.1) and direction $\phi$ at the origin, the Hough transform makes the mapping $(x, y) \rightarrow(\rho, \phi)$. If the coordinates are not known perfectly, then the mapping produces bands that intersect in an area of the parameter space. A histogram of the parameters is filled and, in principle, peaks in the histogram correspond to actual track trajectories. In practice, statistics are frequently low and the histograms are cleaned by removing bins with few entries. The remaining bins are taken to be track candidates and converted to templates. A template is a candidate for one track. The 2D Kalman filter is then applied to select tracks from the templates. An example of this technique is demonstrated in figure 4.1. More details of the Kalman filtering method can be found in references [34, 35].

\subsubsection{Alternate Algorithm}

The other algorithm is known as the alternate algorithm (AA) [36]. This algorithm begins by constructing a track hypothesis from 3 SMT hits. The selection begins at the innermost layer and works outward. The first measurement can be any hit in the 
(a)
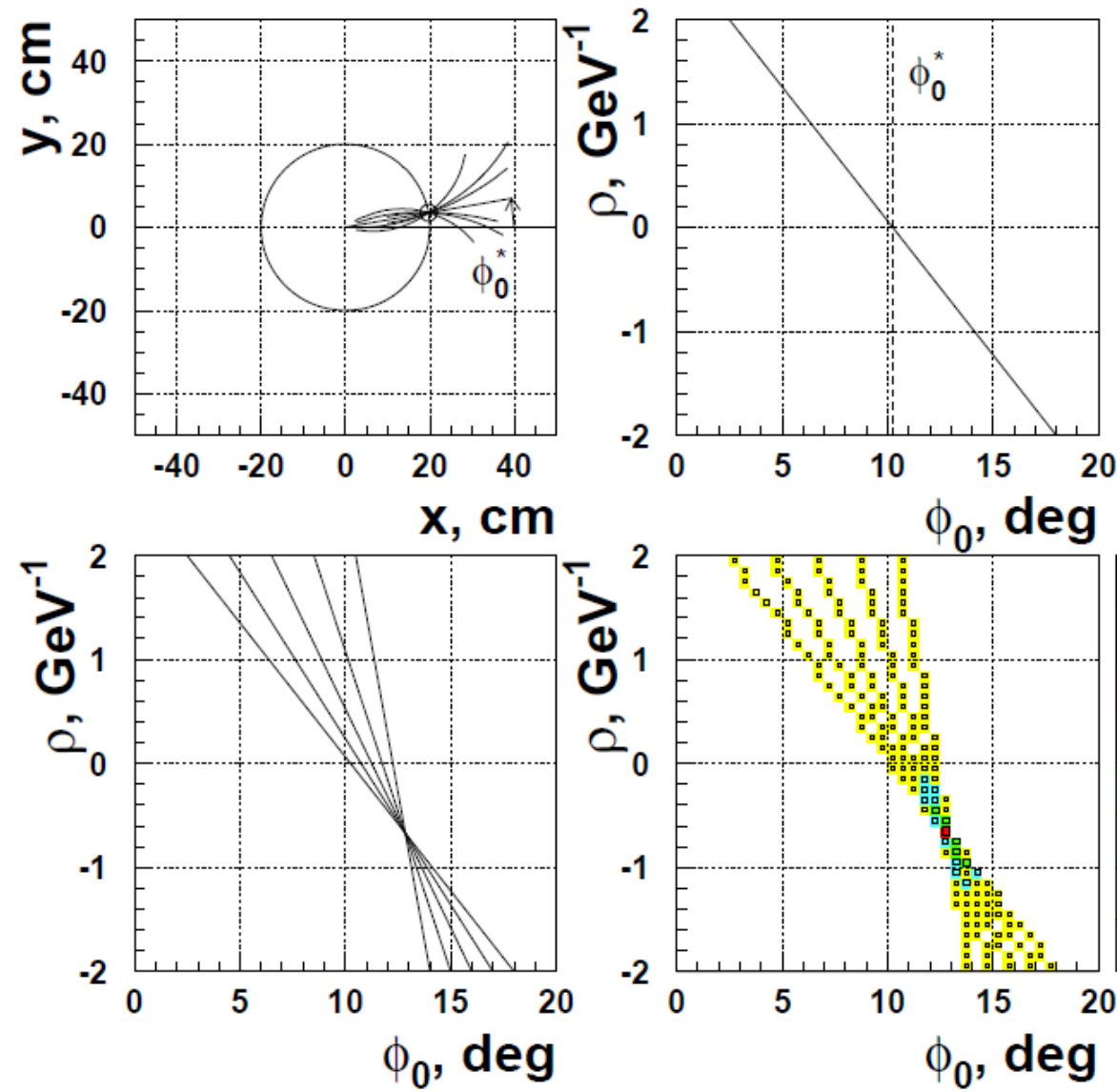

(c)

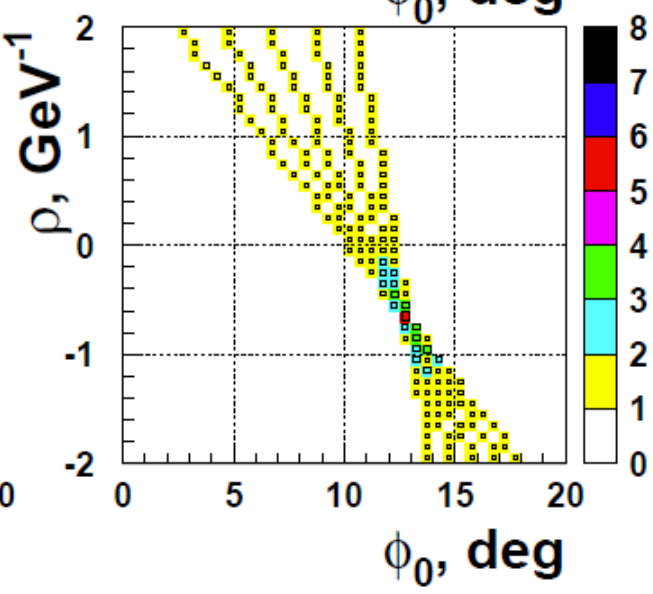

(d)

Figure 4.1: Example of the histogram track finding technique for a single $1.5 \mathrm{GeV}$ track coming from the center of the detector and producing 5 hits. Plot (a) shows the family of trajectories containing a given hit. Plot (b) shows the line in Hough transformed space. Plot (c) shows curves from different hits intersecting at one point corresponding to the track parameters. Plot $(\mathrm{d})$ shows the peak in the $(\rho, \phi)$ histogram which corresponds to the intersection of the lines in parameter space [33]. 
the SMT. The second is selected from any following layer, provided the axial angle between it and the first, as seen from the beam spot, is less than $\Delta \phi=0.08$. The third is also selected from any following layer, provided it falls on a radius greater than $30 \mathrm{~cm}$ and its axial impact parameter with respect to the beam spot is less than $2.5 \mathrm{~cm}$. The track hypothesis must have $\chi^{2}<16$. The track is extrapolated to subsequent layers and hits are added as long as the overall $\chi^{2}$ remains less than 16. A certain number of misses, or layers without any hits, are allowed. The track hypotheses are ordered by the total number of hits, then by fewest number of misses (if the number of hits are equal), and finally by $\chi^{2}$ (if the number of hits and misses are equal).

The same procedure is applied to track hypotheses originating with three CFT hits. However, to reduce the huge number of combinatorics, tracks are required to pass near a primary-vertex as determined by SMT tracks.

\section{$4.2 \quad$ Vertexing}

There are two main classes of vertices: primary-vertices and secondary-vertices. The primary-vertex is the $p \bar{p}$ interaction point and the point of creation for the heavy resonances considered in this analysis, such as the Higgs boson. Particles that are created at the primary vertex may travel some distance before decaying and producing a secondary-vertex. Vertices of either type are found by locating the intersection of two or more tracks. 


\subsubsection{The Primary-Vertex}

Because collisions at the Tevatron occur between bunches of protons and antiprotons, there may be more than one $p \bar{p}$ interaction. However, the hard-scatter event is labeled as the primary-vertex while the other, softer $p \bar{p}$ interactions are known as minimumbias. It is important to identify the primary-vertex in order to fully reconstruct the event. To accomplish this, the adaptive primary-vertex algorithm (AA) is employed [37]. The AA algorithm is implemented as an iterative Kalman filter. In the first pass, all tracks with $p_{T}>0.5 \mathrm{GeV}$ are extrapolated to a common vertex which is determined with the Kalman filter vertex fitting algorithm, using a tear-down approach. There is no SMT hit requirement placed on the candidate tracks. In the tear-down approach, tracks with the highest $\chi^{2}$ contribution to the vertex are removed in turn, until the total $\chi^{2}$ per degree of freedom is smaller than 10. In the second pass, tracks are selected according to their distance of closest approach (dca) to the beam spot and are required to have $d c a<5 \sigma$ where the beam position is computed using the previously measured beam position and error.

The hard-scatter vertex is then selected by assigning to each track a weight given by the following function:

$$
w_{i}=\frac{1}{1+e^{\left(\chi_{i}^{2}-\chi_{c u t o f f}^{2}\right) / 2 T}}
$$

where $\chi_{i}^{2}$ is the contribution to the overall $\chi^{2}$ from track i while $\chi_{\text {cutoff }}^{2}$ and $\mathrm{T}$ are tunable parameters. The optimal values are found to be $\chi_{\text {cutoff }}^{2}=4$ and $T=3$ [37]. The algorithm starts by setting all weights to $1, w_{i}=1$ and then fitting the primaryvertex track candidates using the Kalman filter. The weights are then updated using the new primary-vertex and each track weight, $w_{i}$, is recomputed. If $w_{i}<10^{-6}$ 
the track is eliminated from the fit. The steps are repeated until convergence of the weights is achieved. Convergence occurs when the maximum change in track weights is less than $10^{-4}$.

Once a list of primary-vertices is created, the hard-scatter must be selected. Minimum bias events have low $p_{T}$ tracks, therefore a probability is assigned based on $\log _{10}\left(p_{T}\right)$ for tracks associated with a minimum bias interaction. Individual track probabilities are combined into a total probability for each vertex and the one with the lowest probability to be a minimum bias vertex is selected as the hard-scatter, primary-vertex.

Events are rejected if the $\mathrm{z}$ position of the primary-vertex is not within $35 \mathrm{~cm}$ of the center of the detector. There is not a enough detector volume to properly reconstruct tracks when the primary-vertex is located at high $\mathrm{z}$.

\subsubsection{Secondary-Vertices}

Secondary-vertices are the most important physics object for this analysis. Consequently, accurate secondary-vertex reconstruction is of primary importance.

Events with more than 3 minimum bias vertices do not undergo secondary-vertex reconstruction due to the resulting large track-multiplicity and the subsequent high coincidence rate; when there are a very large number of tracks in an event, the probability is high for two or more tracks to point back to a common vertex because of a coincidence rather than an actual interaction between the two particles which produced the tracks.

Secondary-vertices are reconstructed in a similar fashion as the primary-vertex; the algorithm is based on the Kalman filter fitting technique but using a build-up 
approach. The details of the algorithm are described in reference [38], what follows is a discussion of the criteria for secondary-vertex selection and for the selection of the tracks which are used to construct the vertices. Certain modifications were made to the default algorithm in order to construct secondary-vertices at large radial distances from the primary-vertex.

The first step is to find track clusters. All tracks in a cluster must fall within a cone of size $\Delta R=\sqrt{\Delta \phi^{2}+\Delta \eta^{2}}=0.5$. The tracks must have $p_{T}>0.5 \mathrm{GeV}$ and $\chi^{2}<10$ and cannot be associated with the primary-vertex nor with any minimum bias vertices. The secondary-vertex reconstruction algorithm does not require any SMT hits because this analysis is looking for very highly displaced vertices that may occur at large radial distances. Candidate tracks are further required to have a distance of closest approach to the primary-vertex, $d c a<10 \mathrm{~cm}$ in the $\mathrm{x}-\mathrm{y}$ plane and in the $\mathrm{z}$ direction and a significance of $d c a>3 \sigma$.

In the build-up approach, all two-track combinations within a track cluster are selected as seeds so long as the total $\chi^{2} / n d o f$ of the track cluster with respect to the seed is less than 500. Tracks are then attached to the seed so long as the $\chi^{2} / n d o f$ contribution is smaller than 250. All tracks are used in this step, not only those associated with the cluster. The secondary-vertex location and $\chi^{2}$ are computed by a Kalman filter fitter.

The final step consists of selecting the secondary-vertex from among the candidates. All vertices must have at least 2 tracks, a decay length significance of at least $5 \sigma$, and a collinearity of at least $0.5^{1}$. Secondary-vertices are also not allowed to share tracks. If a track is shared, the "best" vertex keeps the track while the other vertex is

\footnotetext{
${ }^{1}$ Secondary-vertex collinearity is defined as the cosine of the angle between the vector sum of the momentum of the decay products and the direction the parent. Refer to section 6.1.
} 
removed. The result is a list of candidate secondary-vertices for a given track cluster. The final secondary-vertex is then selected as the best one in the cluster. Between two vertices, the best vertex is defined as the one with the smaller opening angle and the smaller $\chi^{2} / n d o f$ (per degree of freedom). An event may contain an arbitrary number of secondary-vertices.

The secondary-vertex invariant mass is an important quantity which is used later in the analysis. The invariant mass is calculated from the four-momenta of the outgoing particle tracks, however the identity, and therefore the mass, of the particles which gives rise to the tracks is not known. Particles which create tracks in the SMT and CFT are typically pions, kaons and protons. Pions are the most frequently produced particles that travel far enough to create tracks. A weighted average of the three is used, corresponding to a mass of $0.25 \mathrm{GeV}$.

\subsection{Jets}

Jet do not play a prominent role in this analysis; they are only used for triggering and for background normalization. Nonetheless, it is important to understand how jets are reconstructed. Figure 4.2 shows a sketch of the evolution of a parton (quark

or gluon) created in a $p \bar{p}$ hard scatter which then hadronizes into particles that travel through the detector and form a jet which is measured as energy in the calorimeter. This section discusses the quality requirements which are used to select good jets and the calibration that is applied to correct for detector and physical effects. 


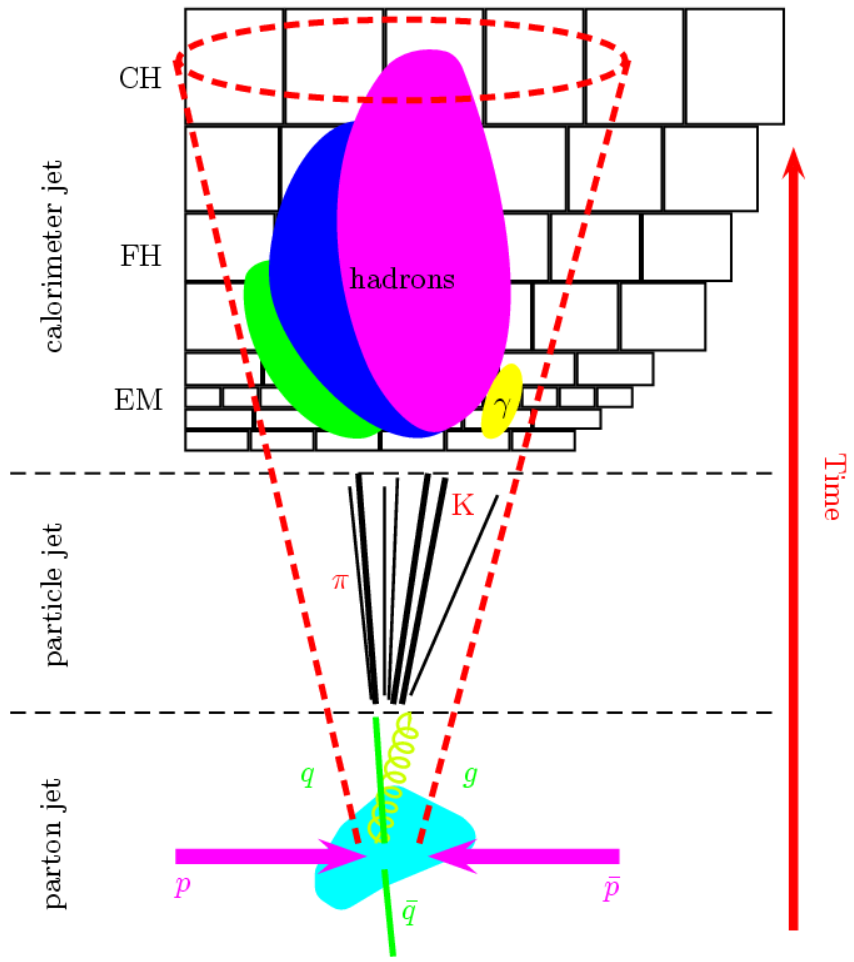

Figure 4.2: A sketch of the evolution from the hard-scatter parton to a jet in the calorimeter [39]. 


\subsubsection{Reconstruction}

Jets are reconstructed with the Run II Cone Algorithm described in reference [20]. The algorithm starts by constructing a $p_{T}$ ordered list of seeds; towers in the calorimeter with $E_{T}>0.5 \mathrm{GeV}$. All of the towers with an energy of at least $1 \mathrm{MeV}$ and within a cone of radius 0.3 in $\eta$ and $\phi$ of the seed tower are then summed to form a precluster. Whenever a tower is added to a pre-cluster it is removed from the list of seeds. Preclusters composed of only one tower or with total $E_{T}<1 \mathrm{GeV}$ are removed from the list of preclusters.

In the next step, the energy of all the towers in a cone of radius $0.5^{2}$ centered on the seed is summed and the center of the new cone is taken to be the $E_{T}$ weighted geometric center of the midpoints of each calorimeter tower. The $E_{T}$ sum is performed again, around the new center and this process is iterated until a stable cone axis is found. Stable cones are called proto-jets.

At this point, the algorithm is sensitive to soft radiation. Figure 4.3 shows an example in which two jets could be reconstructed as one in the presence of soft radiation between two high $E_{T}$ clusters. To overcome this limitation, the midpoints in $\eta-\phi$ between all pairs of proto-jets are taken as seeds and iterated in the same way as the previous seeds.

Duplicate proto-jets are removed as well as those with a total $E_{T}<3 \mathrm{GeV}$. Protojets with overlapping regions are either merged or split and a new cone axis and $E_{T}$ is computed. If the $E_{T}$ of the overlapping region is greater than half of the $E_{T}$ of either jet, then the two jets are merged, otherwise they are split. Finally, after splitting and

\footnotetext{
${ }^{2}$ The jet cone radius is a variable quantity that can range from 0.3 to 1.0. This analysis uses a radius of 0.5 which is labeled as a "JCCB" jet.
} 

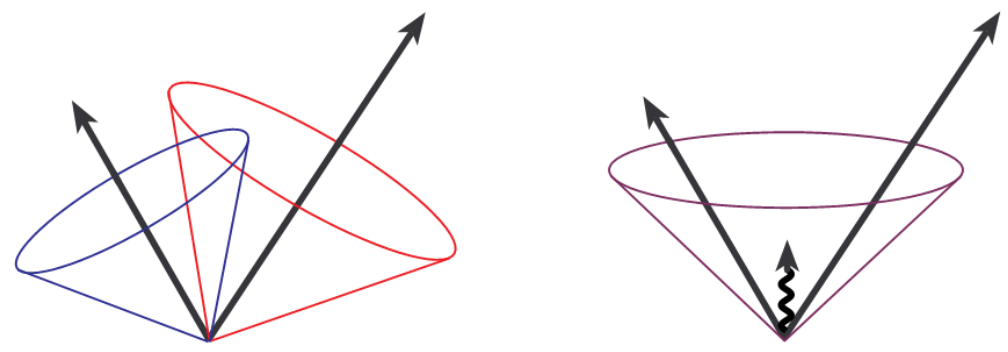

Figure 4.3: An illustration of soft radiation sensitivity in the cone jet algorithm. In this example, jet clustering begins around seed particles, shown here as arrows with length proportional to their energy. The presence of soft radiation between the two jets may cause a merging of the jets which would otherwise not occur [40].

merging, all jets must have $E_{T}>6 \mathrm{GeV}$.

\subsubsection{Jet Selection Criteria}

After jet reconstruction, a series of quality cuts are applied to eliminate fake jets that could be reconstructed from calorimeter noise. The following quality cuts are applied to each reconstructed jet [41]:

- $0.05<$ EM fraction $(\mathrm{EMF})<0.95$

The EM fraction is the ratio of energy deposited in the EM layers to the total energy deposited in the calorimeter. Jets deposit their energy uniformly throughout the calorimeter while electromagnetic objects, photons and electrons, very rarely reach the hadronic portion of the calorimeter. Therefore, EM objects tend to have EMF > 0.95 while jets tend to have EMF of about 0.5, on average.

- coarse hadronic fraction $(\mathrm{CHF})<0.4$

The coarse hadronic portion of the calorimeter is known to be noisy and very 
few jets reach the outer layers which constitute the coarse hadronic calorimeter. As a consequence, reconstructed jets with a high fraction of coarse hadronic energy are rejected.

- hot fraction $<10$

The hot fraction is the ratio of energy in the most energetic cell to that of the next most energetic cell. This cut is intended to reduce the chance of accepting a jet that is reconstructed purely from a hot, or noisy cell in the calorimeter.

- $\mathrm{n} 90>1$

n90 is the minimum number of cells which, together contain $90 \%$ of a jet's energy. If a single cell contains $90 \%$ of the energy of a jet, then it is most likely due to a single hot calorimeter tower or cell.

\subsubsection{Jet Energy Scale}

The goal of the jet energy scale (JES) correction is to correct the energy measured in the calorimeter so that it matches the energy of the particle jets before their interaction with the detector [40]. The JES does not attempt to correct for energy from hard gluon radiation found at very large angles with respect to the original parton direction. The corrected energy is given by the following equation and the terms are described below [39]:

$$
E_{j e t}^{c o r r}=\frac{E_{j e t}^{\text {uncorr }}-E_{\text {offset }}}{F_{\eta} \times R \times S}
$$

- $E_{\text {offset }}$ 
The offset energy $\left(E_{\text {offset }}\right)$ is energy which is not due to the hard scatter such as electronic noise or multiple proton interactions (minimum bias events). This is subtracted from the uncorrected energy of the reconstructed jet.

- $F_{\eta}$

The relative response correction $\left(F_{\eta}\right)$ corrects for the energy response of the calorimeter as a function of jet pseudo-rapidity, $\eta$.

- $R$

The absolute response correction $(R)$ accounts for effects such as uninstrumented detector regions and lower calorimeter response to hadrons as compared to EM objects.

- $S$

The showering correction $(S)$ takes into account energy which is deposited outof-cone. Particles inside a jet cone may deposit energy outside of the reconstructed cone radius or particles which are outside the jet may deposit energy within the reconstructed cone radius. This effect results from shower development in the calorimeter which may cause particles to deposit energy in or out of the cone or from the magnetic field bending particles.

\subsection{Muons}

As with jets, muons are not directly used in this analysis except for the purpose of triggering. Muons are reconstructed by the inner detectors and by the outer muon system. A muon candidate is classified by two parameters: the muon type and the 
muon quality. This analysis uses medium quality muons of type nseg $=3$ which are defined below [42]:

- $\operatorname{nseg}=3$ :

Muons are reconstructed in the $\mathrm{A}$ and $\mathrm{BC}$ layers of the muon system and matched to a central track. A central track is one that is reconstructed by the inner detector (SMT and CFT).

- medium quality:

The muon must have at least two A layer wire hits and at least one A layer scintillator hit. It must also have at least two BC layer wire hits and at least one $\mathrm{BC}$ scintillator hit (except for central muons with less than four BC wire hits).

Because of the curvature induced by the toroid magnet, the momentum of the muon can be measured from the hits in the three layers of the muon system, however the central detector is able to make a much better measurement.

Cosmic muons are removed by making timing and location cuts. The muon must be observed in all three muon layers within 10 ns of a bunch crossing and muon tracks are required to have $d c a<0.16 \mathrm{~cm}$ from the primary-vertex. The muon must also be matched within $2 \mathrm{~cm}$ of the beamspot.

Finally, to increase the probability of selecting a muon from the decay of a b or c quark, the muon is required to have $p_{T}>4 \mathrm{GeV}$ and it must be matched to a jet within a cone of radius $\Delta R=0.7$. 


\section{Chapter 5}

\section{Data and Simulation}

\subsection{Data}

The dataset which is used in this analysis was collected from April 2002 to August of 2008 during Run II of the Tevatron. In order to maximize the discovery potential of this analysis we do not make any specific trigger requirements. The most frequently used triggers typically require a muon and a jet and are summarized in tables A.1 and A.2 in appendix A.1. We estimate the effect of the combined triggers on our signal in section 5.3.

To analyze the relevant subset of the events collected by the DØ detector we use skims. Muons and jets are frequent features of b-decays which are characteristic of our signal, therefore we make use of the "SKIM_BID" portion of the data. The "SKIM_BID" requires that events meet the following criteria to enhance the fraction of events with a b-quark:

- One medium quality muon [42] with $p_{T}>4 \mathrm{GeV}$. The muon must have hits 
in both the $\mathrm{A}$ and $\mathrm{BC}$ layers of the muon system (nseg=3) and be matched to within $\Delta R<0.7$ to a jet of cone radius 0.5 (JCCB). The cone radius is specified by $\Delta R=\sqrt{(\Delta \phi)^{2}+(\Delta \eta)^{2}}$ with $\phi$ and $\eta$ being the azimuth and pseudo-rapidity. This requirement enhances the fraction of heavy-flavor events due to the decays, $b \rightarrow \mu$ and $b \rightarrow c \rightarrow \mu$.

- At least 2 jets (JCCB), each with $p_{T}>10 \mathrm{GeV}$. All jets are JES corrected.

To ensure good vertex reconstruction we further require the following:

- The PV must be located within $|z|<35 \mathrm{~cm}$ and $r<1 \mathrm{~cm}$ of the center of the beamline.

- Less than three minimum bias vertices. In any given event there is only one primary vertex (as described in section 4.2) but there might be multiple vertices which produce particles near the $p \bar{p}$ interaction point. More minimum bias vertices can produce events that are poorly reconstructed due to the large number of resulting tracks (as described in section 4.2).

There are approximately 50 million events remaining after imposing all data quality and skim requirements. Since the run 2a (p17) and run 2b (p20) datasets are sufficiently different they are analyzed separately (refer to appendix B).

The total luminosity of the sample after data quality requirements is $3.65 \mathrm{fb}^{-1}$ as measured with the standard luminosity tools [43]. The default dataset definitions and their luminosities are summarized in table 5.1. The effective luminosity of the data used in the analysis is $2.7 \mathrm{fb}^{-1}$ where the reduction results from the minimum bias requirement (see fig. 5.1). 


\begin{tabular}{c|c|c|c}
\hline run & dates & data set name & $\begin{array}{c}\mathcal{L} \\
\left(\mathrm{pb} b^{-1}\right)\end{array}$ \\
\hline \hline 2a & Apr. & CSskim-MUinclusive-PASS3-p17.09.06b & 120.92 \\
& $20,2002-$ & & \\
& Feb. 22, & & \\
& 2006 & CSskim-MUinclusive-PASS3-p17.09.06 & 97.27 \\
& & CSskim-MUinclusive-PASS3-p17.09.03 & 842.66 \\
\hline 2b & Jun. 9, 2006 & CSskim-MUinclusive-PASS2-p21.03.00-allfix2007 & 1214.77 \\
& - Aug. 4, & & \\
& 2007 & CSskim-MUinclusive-PASS4-p21.08.00-allfix2008 & 404.34 \\
& Oct. 28, & & 34.19 \\
& $2007-$ & CSskim-MUinclusive-PASS4-p21.08.00-p20.12.01 & 34.00 \\
& - & CSskim-MUinclusive-PASS4-p21.08.00-p20.12.02 & 934.48 \\
\hline & Aug., 2008 & TOTAL & 3648.63 \\
\hline
\end{tabular}

Table 5.1: The datasets used in this analysis. The integrated luminosity $\left(\int \mathcal{L}\right)$ is for "good quality" as defined by by the SMT, CFT, Calorimeter and Muon groups [43].
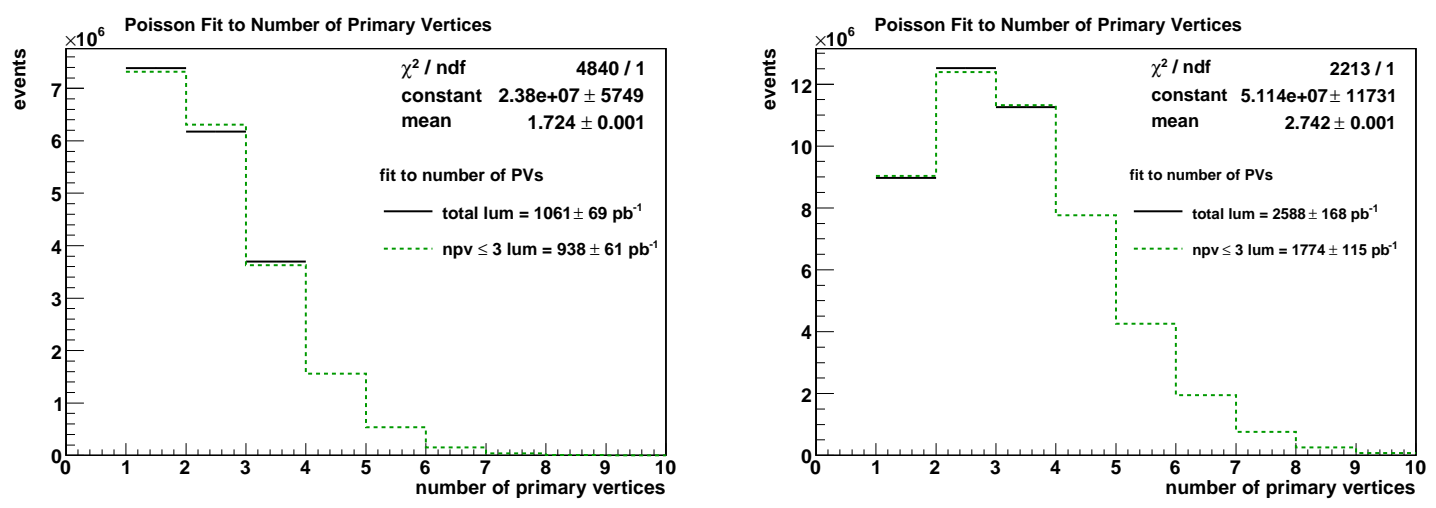

Figure 5.1: A fit of the number of primary vertices to a Poisson distribution to estimate the luminosity of the dataset after requiring 3 or fewer primary vertices per event. Green is the fitted line and black is the data used in the analysis. The uncertainty includes both the standard luminosity uncertainty as well as the uncertainty from the fit. The average instantaneous luminosity in run 2b (p20; on the right) was higher than in run $2 \mathrm{a}$ (p17; on the left) which produced more primary-vertices per event. As a result, more data was cut in p20 than in p17. The total integrated luminosity of the two samples is $2712 \mathrm{fb}^{-1}$. 
Vertexing is accomplished by the method described in section 4.2. Some modifications of the vertexing parameters are necessary to handle tracks at large radii. The impact parameter requirement is increased from $0.15 \mathrm{~cm}$ to $10 \mathrm{~cm}$ in the xy-plane and $0.4 \mathrm{~cm}$ to $10 \mathrm{~cm}$ in the z-plane. We also remove a hard cut which excludes vertices located at $L x y \geq 2.6 \mathrm{~cm}$ or $L z \geq 5 \mathrm{~cm}$ with respect to the primary-vertex. The maximum allowed $\chi^{2}$ for vertex reconstruction and track fitting is increased from 15 to 500. Refer to section 4.2 .

After reconstruction, both data and MC are processed through a custom ntuplegenerating package. The jet energy scale corrections from section 4.3 are applied to all jets.

\subsection{Monte Carlo Techniques}

In general, a Monte Carlo program simulates a given process by repeated random sampling of a known distribution. This is especially useful when the underlying theory is so complicated that an exact solution is not feasible or the underlying theory is inherently random. Physical processes in quantum mechanics occur randomly, but with a known distribution so Monte Carlo simulation is especially well suited for modeling.

Simulated events at $\mathrm{D} \varnothing$ for this analysis are produced through the following steps: (1) physics events are generated by the Pythia [44] and AlPGEn Monte Carlo software programs, (2) the events are run through a full detector simulation to replicate the response of the $\mathrm{D} \varnothing$ detector, and (3) the events are reconstructed using the same algorithm that reconstructs data events as discussed in section 4. Signal and back- 
ground events are generated by Monte Carlo and both are further fortified with zero bias events; actual background events from $p \bar{p}$ collisions recorded by the $\mathrm{D} \emptyset$ detector. The simulated signal and background events are then used to determine the optimal selection criteria which will be applied to the data so the hypothetical signal can be extracted.

\subsubsection{Background Monte-Carlo Samples}

The mc_runjob software package is used to process both background and signal MC through the full GEANT 3-based D $\varnothing$ detector simulation. Events are then reconstructed with the same software used to reconstruct data events. The same primary-vertex $(\mathrm{PV})$ location requirements are also imposed on all $\mathrm{MC}$ samples and jet energy scale corrections are applied to all jets. The $\mathrm{D} \varnothing \mathrm{p} 17.09 .01$ software release is used throughout.

\subsubsection{Background}

The background model is composed of two distinct MC samples:

- 2.1 million events of $b \bar{b}+X$ and $c \bar{c}+X$ monte-carlo. AlPGEN is used to generate the final-state partons while PYTHIA performs the showering and decay of the final-state partons.

- 2.4 million events of QCD inclusive MC is generated by PYTHIA with parton transverse momenta in the range, $10<p_{T}<980 \mathrm{GeV}$.

The samples of $b \bar{b}$ and $c \bar{c} \mathrm{MC}$ are used to increase the statistical power of the background model and they should behave identically as the QCD MC at long decay 
lengths (far from the beamline). A check is performed to insure that both samples separately agree. Figure 5.2 shows good agreement between the two MC samples for leading jet $p_{T}$, secondary-vertex (SV) collinearity, SV mass and SV track multiplicity. The SV variables are used to distinguish background from signal.

The events of the combined sample are weighted so that the distribution of leading jet $p_{T}$ and number of primary-vertices agree with the data sample as demonstrated in figure 5.3 and 5.4. Since the luminosity profiles of run 2a (p17) and run 2b (p20) are sufficiently different, Monte-Carlo events are weighted to agree with p17 or p20 and the two datasets are analyzed separately. Effectively there are two Monte-Carlo sets; one which is weighted to agree with p17 and one which is weighted to agree with p20, although the same events are used in each set.

The skim requirements outlined in section 5.1 are not imposed since QCD events typically do not contain a muon and the skim requirement would have the unwanted effect of reducing the number of background events by about $90 \%$. This does not introduce a bias because muons are not used beyond the trigger. From here on, the background $\mathrm{MC}$ is labeled "QCD" in figures.

\subsubsection{Signal Modeling}

Signal events are generated by PYTHIA (version 6.319) in which the initial Higgs boson is created via gluon fusion, $g g \rightarrow H$ with the Higgs decaying to an $A^{0}$ pair where the $A^{0}$ is a pseudo-scalar, supersymmetric Higgs that represents a v-hadron. Each $A^{0}$ is subsequently forced to decay to a pair of b-quarks. The mass of the Higgs, the mass of the $A^{0}$ and the decay length of the $A^{0}$ are varied. Events are overlaid with an average of 1.0 zero-bias (ie. random) event from data. Signal MC events are 

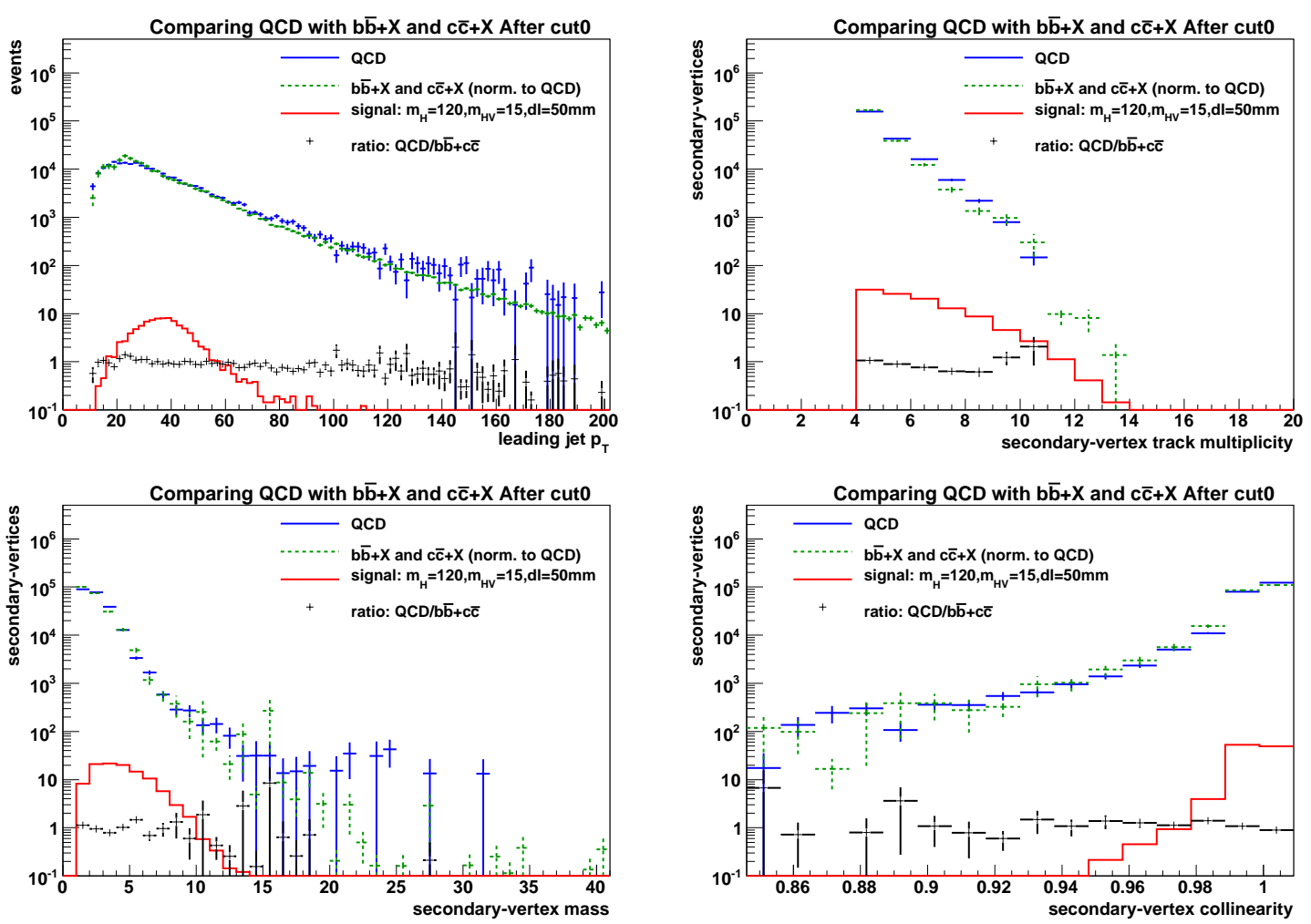

Figure 5.2: Agreement between QCD MC and $b \bar{b}+X$ and $c \bar{c}+X$ MC. All samples are normalized to each other. The "cut0" pre-selection criteria requires that each SV must be reconstructed with $\chi^{2}<20$, have a track multiplicity greater than 4 and be located at a distance of at least $1.6 \mathrm{~cm}$ in the transverse plane. The cut0 pre-selection is discussed in section 6.2. 

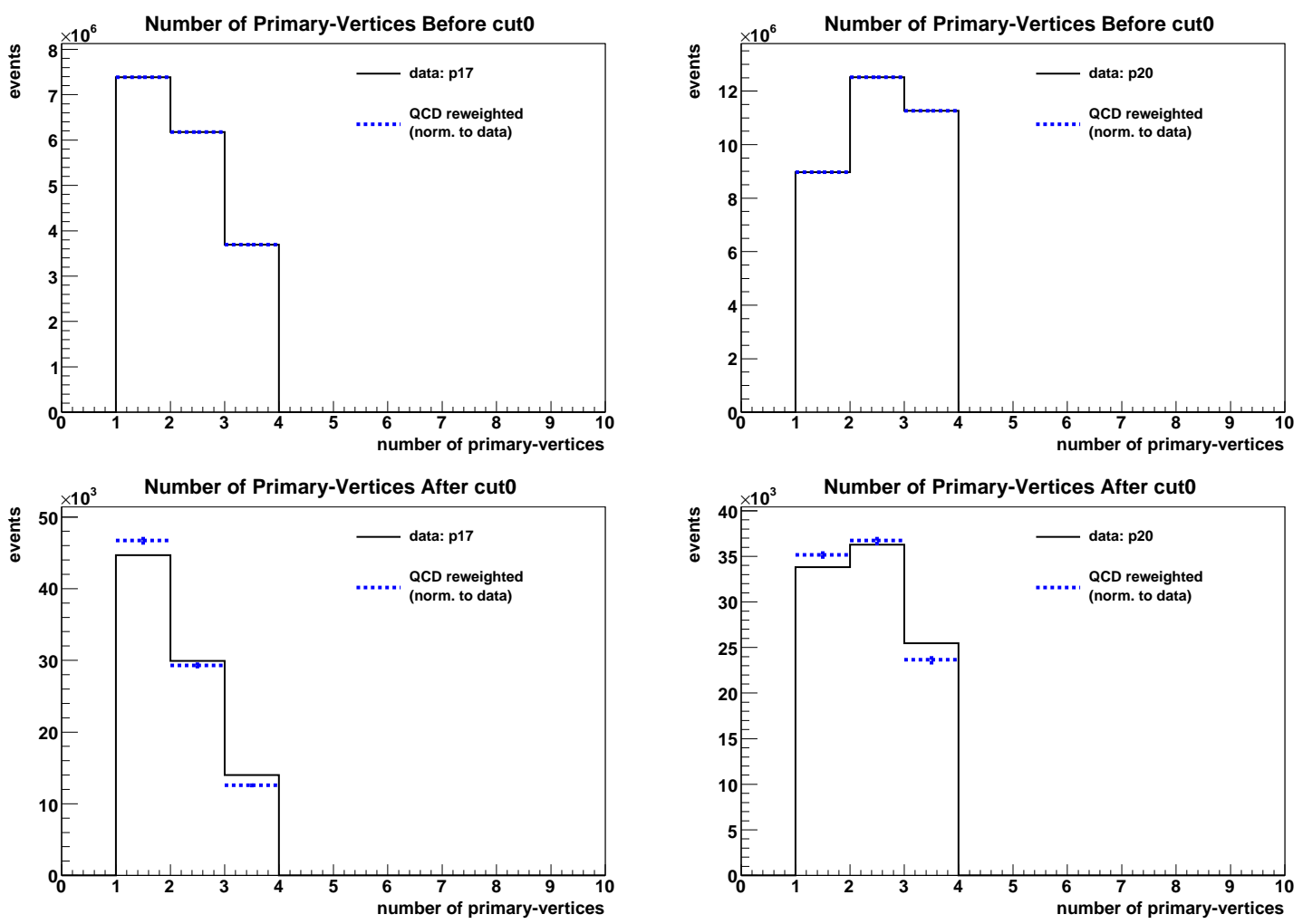

Figure 5.3: QCD MC after reweighting to run 2a (p17; on the left) and run 2b (p20; on the right) data. All samples are normalized to each other. The "cut0" pre-selection criterion is defined in section 6.2. 

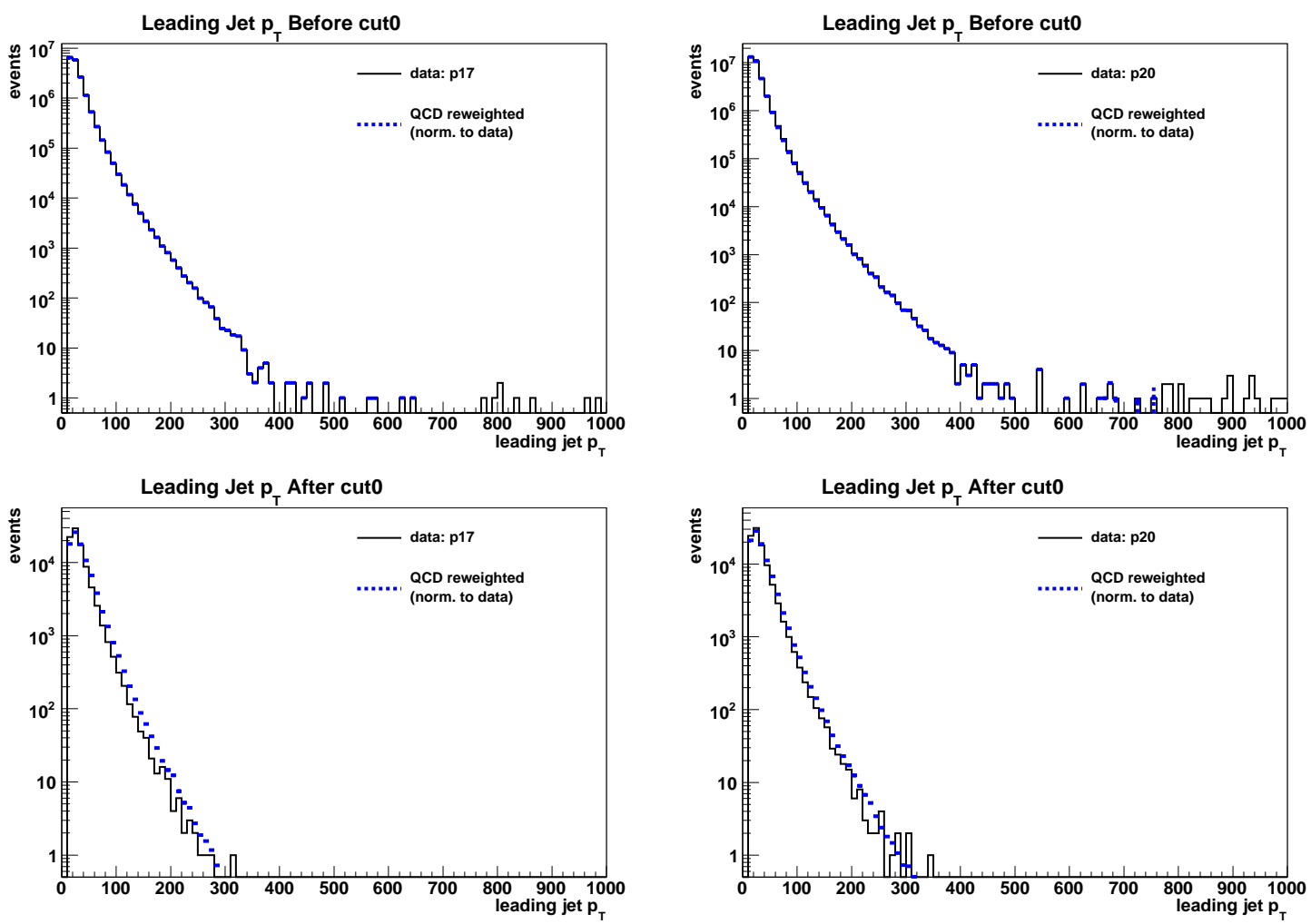

Figure 5.4: QCD MC before and after reweighting to run 2a (p17; on the left) and run $2 \mathrm{~b}$ (p20; on the right) data. All samples are normalized to each other. The "cut0" pre-selection criterion is defined in section 6.2. 


\begin{tabular}{c|c|c|c|c}
\hline$m_{H}(\mathrm{GeV})$ & $m_{H V}(\mathrm{GeV})$ & $c \tau(\mathrm{mm})$ & $\mathrm{SM} \sigma_{g g \rightarrow H}(\mathrm{pb})$ & $N_{\text {generated }}$ \\
\hline \hline 90 & 15 & 50 & 2.0 & 135,788 \\
90 & 40 & 50 & 2.0 & 108,871 \\
120 & 15 & 25 & 1.0 & 95,168 \\
120 & 15 & 50 & 1.0 & 116,738 \\
120 & 15 & 100 & 1.0 & 140,251 \\
120 & 40 & 50 & 1.0 & 119,750 \\
200 & 15 & 50 & 0.2 & 101,402 \\
200 & 40 & 50 & 0.2 & 103,213 \\
\hline
\end{tabular}

Table 5.2: Summary of the signal hypotheses used in this analysis and number of events generated.

required to pass the same skim and PV location requirements as data (refer to section 5.1). We look for a Higgs boson with $m_{H}=200 \mathrm{GeV}, m_{H}=120 \mathrm{GeV}$ and $m_{H}=90$ $\mathrm{GeV}$ which is just above the most general LEP limit. Table 5.2 summarizes the eight signal hypotheses which are generated and analyzed.

To calculate the expected number of signal events, we scale each sample by,

$$
\text { scale }=\frac{\sigma_{g g \rightarrow H} \cdot \int \mathcal{L} \cdot \epsilon_{\text {all triggers }}}{N_{\text {generated }}}
$$

where $\sigma_{g g \rightarrow H}$ is taken to be the SM Higgs production cross section from reference [45], $\int \mathcal{L}$ is the integrated luminosity found in section $5.1, \epsilon_{\text {trigger }}$ is the efficiency for triggering on a signal event as described in section 5.3 and $N_{\text {generated }}$ is the number of generated MC events. We also assume a $100 \%$ branching ratio (BR) of $\pi_{v} \rightarrow b \bar{b}$.

\subsection{Trigger Efficiency}

The probability for a signal event to pass the combined single muon triggers is found with the muid_eff package. The muid_eff package parametrizes trigger efficiencies 
for a given type of offline muon in terms of muon kinematic quantities (we require a medium quality, nseg $=3$ muon as discussed in section 5.1). Trigger efficiencies are found by using the tag and probe method [42] on dimuon samples. The tagged muon must be of loose quality, it must be matched to a central track and it must satisfy a single muon trigger. The probe muon must be of medium quality and it must satisfy certain angular requirements in relation to the probe track. The probe muon is then matched to a muon trigger object to estimate the muon trigger efficiency.

Similar to the muon, the probability for a signal event to pass the combined single jet triggers is found with the jetid_eff package. Jet efficiency uses the tag-and-probe method on dijet events and parametrizes trigger efficiencies for "good" offline jets [46].

Appendix A.2 contains the locations of the files used for the parametrizations and appendix A.3 shows plots of the trigger efficiencies.

As mentioned in section 5.1, we place no specific trigger requirements on the data. Therefore it is necessary to estimate the efficiency of all the triggers on our signal by using the data itself . The number of events passing a single trigger is given by,

$$
N_{\text {trigger }}^{\text {signal }}=\int \mathcal{L}{ }_{\text {trigger }} \cdot \sigma_{\text {signal }} \cdot \epsilon_{\text {trigger }}^{\text {signal }}
$$

while the number of events passing all triggers is,

$$
\begin{aligned}
& N_{\text {alltriggers }}^{\text {signal }}=\int \mathcal{L}_{\text {all triggers }} \cdot \sigma_{\text {signal }} \cdot \epsilon_{\text {all triggers }}^{\text {signal }} \\
& \Longrightarrow \epsilon_{\text {alltriggers }}^{\text {signal }}=\epsilon_{\text {trigger }}^{\text {signal }} \cdot \frac{N_{\text {all }}^{\text {signal }}}{N_{\text {trigger }}^{\text {signal }}} \cdot \frac{\int \mathcal{L}_{\text {trigger }}}{\int \mathcal{L}_{\text {alltriggers }}}
\end{aligned}
$$

where $\sigma_{\text {signal }}$ is the signal production cross-section, $\epsilon_{\text {trigger }}^{\text {signal }}$ is the efficiency of a single 
trigger to pass the signal and $\epsilon_{\text {altrigger }}^{\text {signal }}$ is the efficiency of the combined triggers to pass the signal. The production cross-section for signal is taken to be the same as the SM Higgs production cross-section, $\sigma_{\text {signal }}=\sigma_{g g \rightarrow H}$.

Fundamentally then, the problem is reduced to finding a suitable estimate for the ratio of the number of events passing all the triggers to the number of events passing a single trigger, $\frac{N_{\text {al }}^{\text {signal }}}{N_{\text {trigal }}^{\text {siger }}}$. If our loose selection criteria pick events which are close to signal, at least as far as the trigger is concerned, then we can make the approximation,

$$
\frac{N_{\text {alltriggers }}^{\text {selected }}}{N_{\text {triggect }}^{\text {seled }}} \approx \frac{N_{\text {all }}^{\text {signal }}}{N_{\text {trignger }}^{\text {signal }}}
$$

This is a reasonable approximation because our signal is characterized by the features of the pre-selection criteria.

For the purpose of this calculation we need to select a trigger which both collects a large portion of the data and can be modeled by the muid_eff and jetid_eff software packages [47]. Since Run 2a and Run $2 \mathrm{~b}$ use different trigger lists, two triggers need to be modeled. For Run 2a we select the "MU_2TRK3_L2M0" trigger which acquired $20 \%$ of the data (see table A.1) and has the following requirements at each detector trigger level:

L1 A single muon in the A-region (wide muon region) based on muon scintillators. One Calorimeter JET trigger tower with $E_{T}>5 \mathrm{GeV}$.

L2 One muon of medium quality without $p_{T}$ requirement.

L3 Two tracks with $p_{T}>3 \mathrm{GeV}$.

For Run 2b we select the "MUJ5_MM0_2TK3_VX" trigger which gives rise to 39\% 
of the data (see table A.2) and has the following requirements at each detector trigger level:

L1 A single muon in the all-region $(|\eta|<2.0)$ with tight scintillator and tight wire requirements and no CTT track requirement. Also one jet with $E_{T}>15 \mathrm{GeV}$ and $\mid$ eta $\mid<3.2$.

L2 One muon with $p_{T}>3 \mathrm{GeV}$, of medium quality and no region requirement. Also, one jet with $E_{T}>20 \mathrm{GeV}$.

L3 Two global tracks with $p_{T}>3 \mathrm{GeV}$. Also, one PV with $|z|<35 \mathrm{~cm}$ and one muon with no $p_{T}$ threshold.

We also verify that the trigger does not bias the data for the variables used in this analysis. Figure 5.5 demonstrates good agreement, within statistical fluctuations, between SV multiplicity, collinearity and mass for data collected with the single trigger and all the triggers.

For the selection criteria, we require events with 1 SV having multiplicity $\geq 4$, $2 \mathrm{~d}$ decay length $>1.6 \mathrm{~cm}$ and $\chi^{2}<20$. We also require two offline tracks with $p_{T}>5 \mathrm{GeV}$. Using the muid_eff and jetid_eff packages we estimate the efficiency of the single trigger to pass a signal event. The software package gives efficiencies as a function of detector $\eta, \phi$ and $z$. Tables 5.3 and 5.4 summarize the efficiencies of our two selected triggers at each trigger level.

Next we consider trigger turn-on effects. With respect to the muons that are selected, the trigger is already fully efficient and turn-on effects do not need to be considered (refer to figure 5.6). The jet trigger turn-on is estimated from the ratio of single-trigger leading jet $p_{T}$ to all-trigger leading jet $p_{T}$ as shown in figure 5.6. 

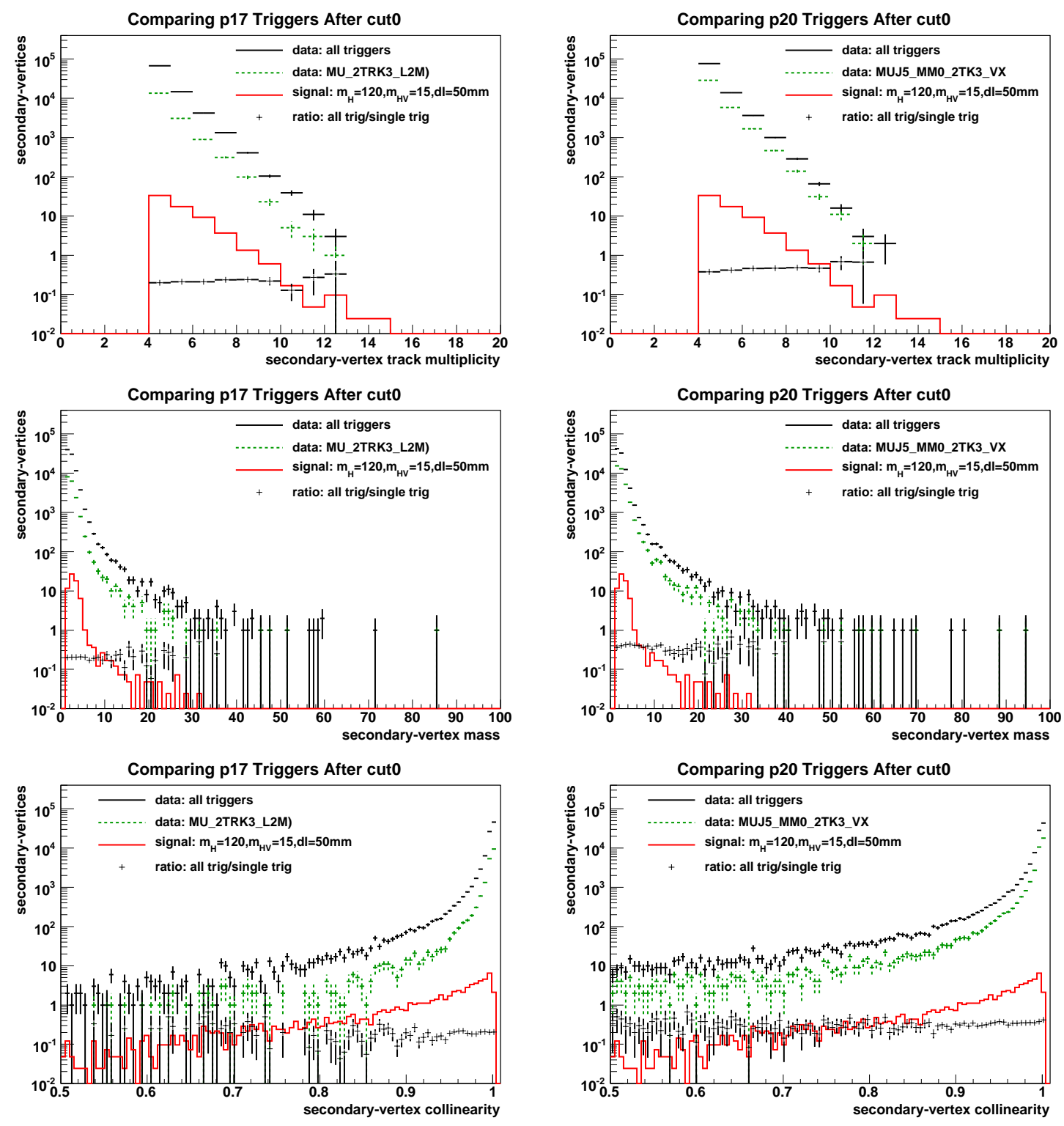

Figure 5.5: A comparison of data collected with the selected single trigger and all triggers. Run $2 \mathrm{a}$ is on the left and Run $2 \mathrm{~b}$ on the right. The $m_{H}=120 \mathrm{GeV}$, $m_{H V}=15 \mathrm{GeV}, \mathrm{dl}=50 \mathrm{~mm}$ signal sample is shown to illustrate the location of signal events. 

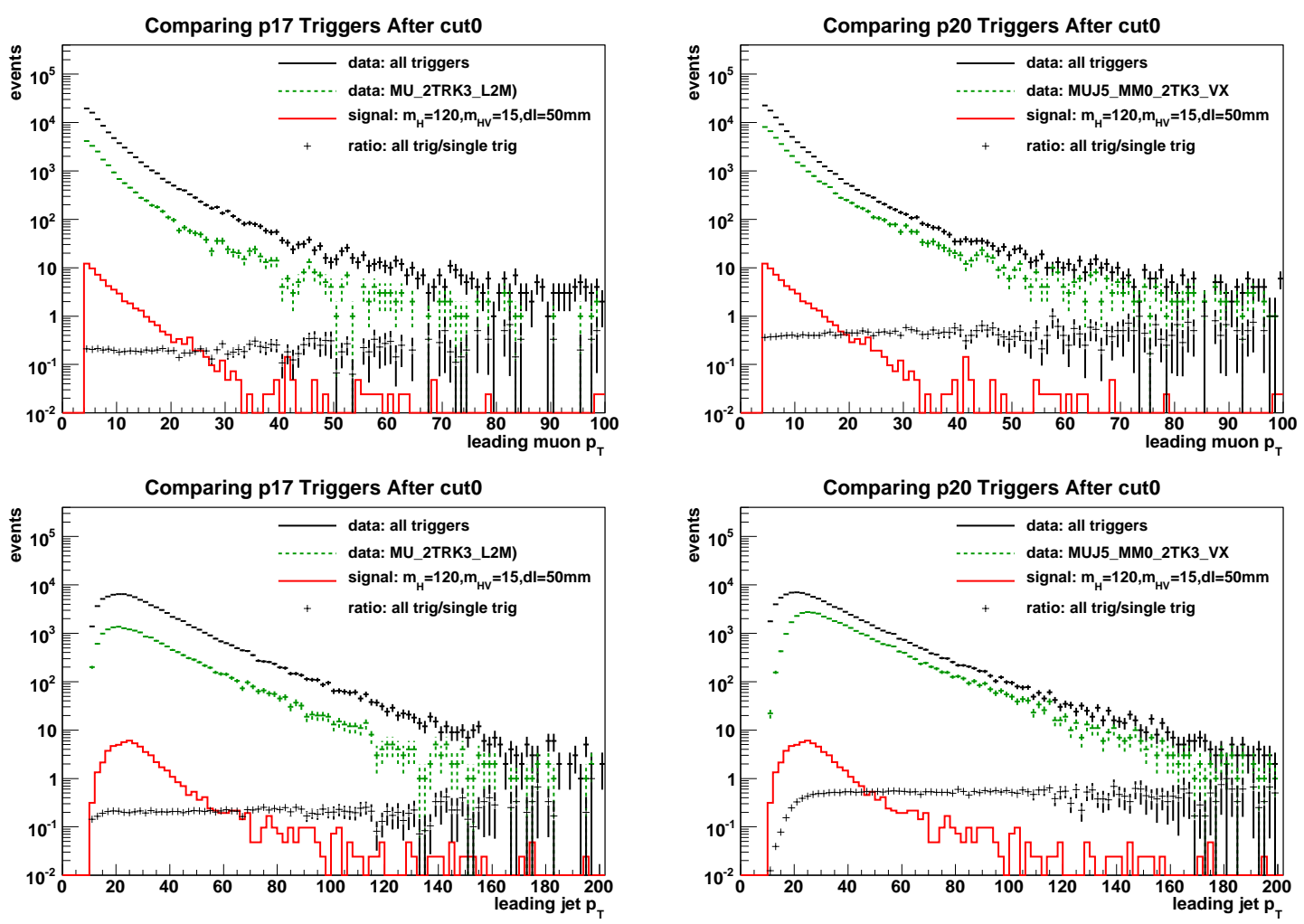

Figure 5.6: The ratio of the number of events from the single trigger to all triggers as a function of leading muon $p_{T}$ and leading jet $p_{T}$. The Run $2 \mathrm{a}$ trigger ratio is on the left and the Run $2 \mathrm{~b}$ ratio is on the right. The $m_{H}=120 \mathrm{GeV}, m_{H V}=15 \mathrm{GeV}$, dl $=$ $50 \mathrm{~mm}$ signal sample is shown to illustrate the location of signal events . The black line is events collected by all the triggers, the red line is signal events, the green line is events collected by the single-trigger, and the line represented by the black cross section is the ratio of the number of all-trigger events to single-trigger events. 


\begin{tabular}{c|c|c|c|c|c}
\hline $\begin{array}{c}\text { signal sample } m_{H}(\mathrm{GeV}), m_{H V} \\
(\mathrm{GeV}), \mathrm{dl}(\mathrm{mm})\end{array}$ & $\begin{array}{c}\text { muon } \\
\text { L1 }\end{array}$ & $\begin{array}{c}\text { jet } \\
\text { L1 }\end{array}$ & $\begin{array}{c}\text { muon } \\
\text { L2 }\end{array}$ & $\begin{array}{c}\text { jet } \\
\text { L2 }\end{array}$ & $\begin{array}{c}\text { track } \\
\text { L3 }\end{array}$ \\
\hline \hline$m_{H}=90, m_{H V}=15, \mathrm{dl}=50$ & $98 \%$ & $62 \%$ & $100 \%$ & $95 \%$ & $95 \%$ \\
$m_{H}=90, m_{H V}=40, \mathrm{dl}=50$ & $99 \%$ & $58 \%$ & $100 \%$ & $88 \%$ & $97 \%$ \\
$m_{H}=120, m_{H V}=15, \mathrm{dl}=25$ & $99 \%$ & $85 \%$ & $100 \%$ & $99 \%$ & $98 \%$ \\
$m_{H}=120, m_{H V}=15, \mathrm{dl}=50$ & $99 \%$ & $86 \%$ & $100 \%$ & $99 \%$ & $97 \%$ \\
$m_{H}=120, m_{H V}=15, \mathrm{dl}=100$ & $99 \%$ & $87 \%$ & $100 \%$ & $99 \%$ & $96 \%$ \\
$m_{H}=120, m_{H V}=40, \mathrm{dl}=50$ & $99 \%$ & $64 \%$ & $100 \%$ & $95 \%$ & $98 \%$ \\
$m_{H}=200, m_{H V}=15, \mathrm{dl}=50$ & $100 \%$ & $97 \%$ & $100 \%$ & $100 \%$ & $98 \%$ \\
$m_{H}=200, m_{H V}=40, \mathrm{dl}=50$ & $100 \%$ & $93 \%$ & $100 \%$ & $100 \%$ & $99 \%$ \\
\hline
\end{tabular}

Table 5.3: The average trigger efficiency of each p20 trigger level for each signal sample. The p20 trigger is MUJ5_MM0_2TK3_VX. Events are required to pass the cut0 pre-selection (refer to table 6.1 for the definition of cut0).

Finally, the result is an estimate of trigger efficiency as a function of leading jet $p_{T}, \eta, \phi$ and $z$ given by, ${ }^{1}$

$$
\begin{gathered}
\epsilon\left(\text { jet }, p_{T}\right)=\epsilon_{\text {trigger }}^{\text {signal }}(\eta, \phi, z) \cdot \frac{N_{\text {all }}^{\text {selected }}}{N_{\text {trigger }}^{\text {selected }}} \cdot \frac{L_{\text {trigger }}}{L_{\text {all triggers }}} \cdot \operatorname{ratio}(j e t, p T) . \\
\epsilon_{\text {run } 2 a}\left(j e t, p_{T}\right)=.94 \cdot \epsilon_{\text {trigger }}^{\text {signal }}(\eta, \phi, z) \cdot \operatorname{ratio}\left(j e t, p_{T}\right) . \\
\epsilon_{\text {run } 2 b}\left(\text { jet }, p_{T}\right)=.96 \cdot \epsilon_{\text {trigger }}^{\text {signal }}(\eta, \phi, z) \cdot \operatorname{ratio}\left(\text { jet }, p_{T}\right) .
\end{gathered}
$$

which is applied to the signal samples for the analysis. Figures 5.7, 5.8 and 5.9 show the trigger efficiency for each signal sample as a function of jet $p_{T}$ while table 5.5 summarizes the average trigger efficiency and uncertainty for the eight signal samples which are studied in this analysis.

\footnotetext{
${ }^{1}$ ratio $\left(j e t p_{T}\right)$ is normalized so that the flat portion of the curve is set to 1 .
} 

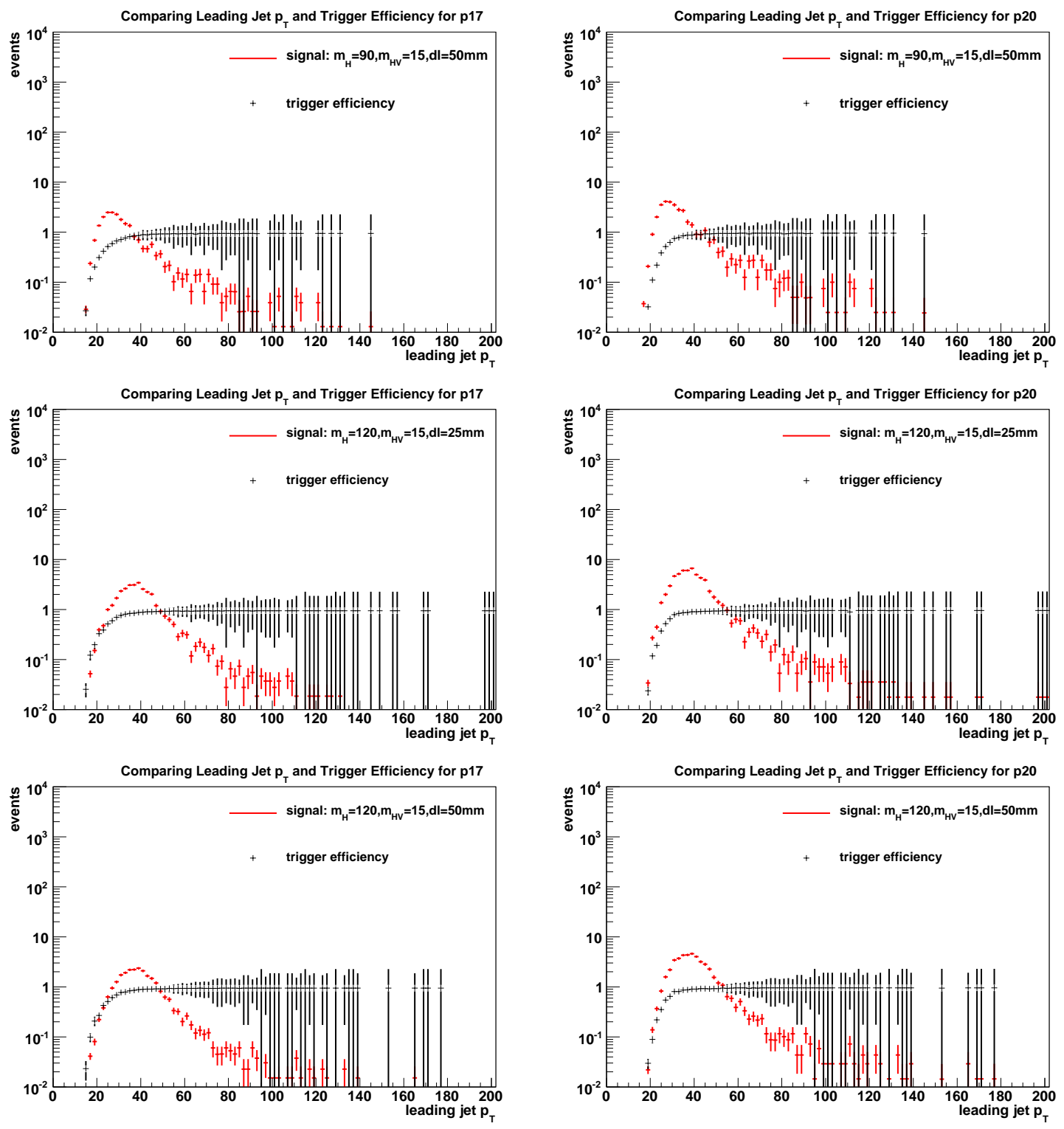

Figure 5.7: Leading jet $p_{T}$ and overall trigger efficiency as a function of leading jet $p_{T}$ for three different signal samples. Plots on the left show the total p17 trigger efficiency while plots on the right show total p20 trigger efficiency. 

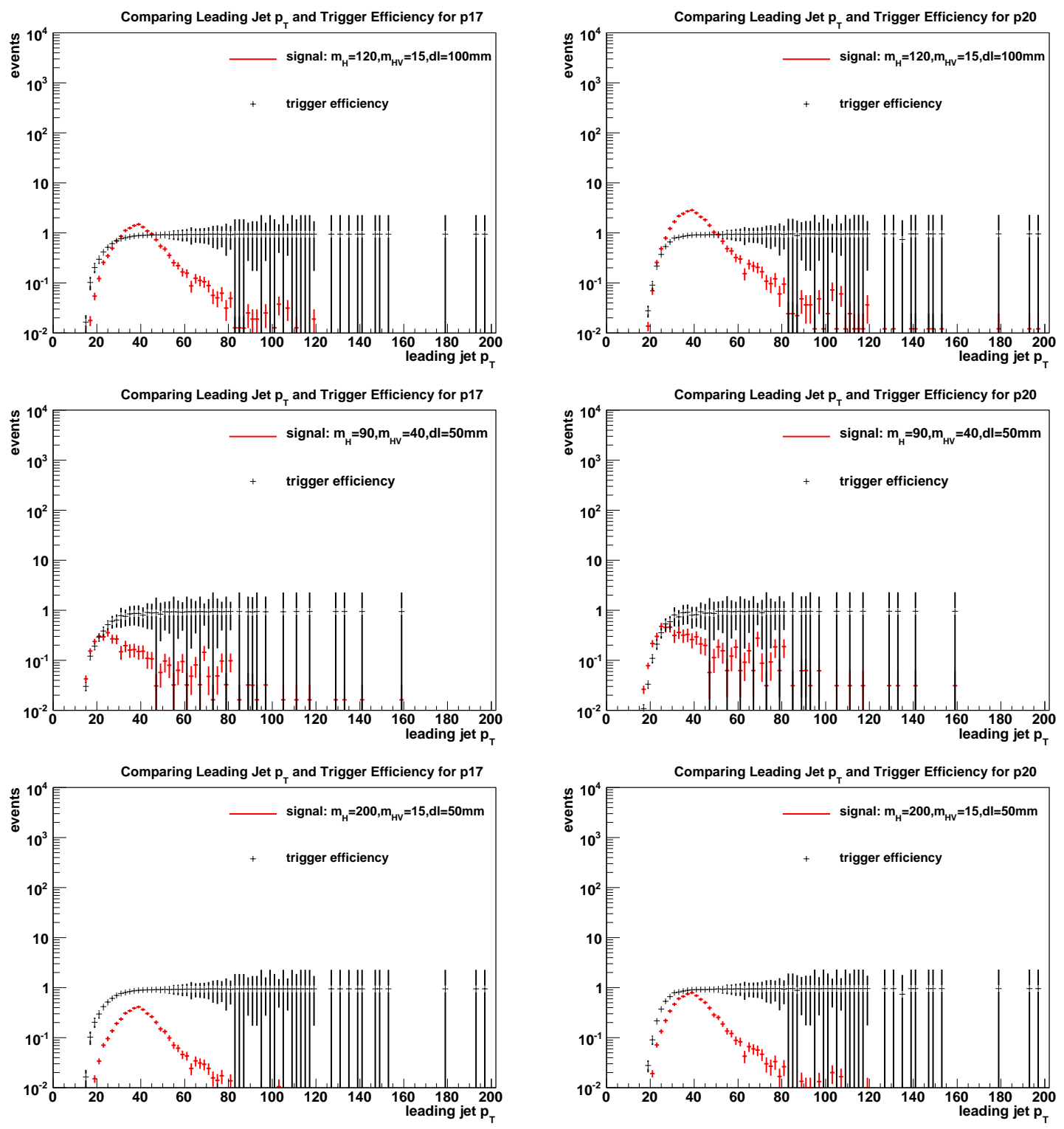

Figure 5.8: Leading jet $p_{T}$ and overall trigger efficiency as a function of leading jet $p_{T}$ for three different signal samples. Plots on the left show the total p17 trigger efficiency while plots on the right show total p20 trigger efficiency. 

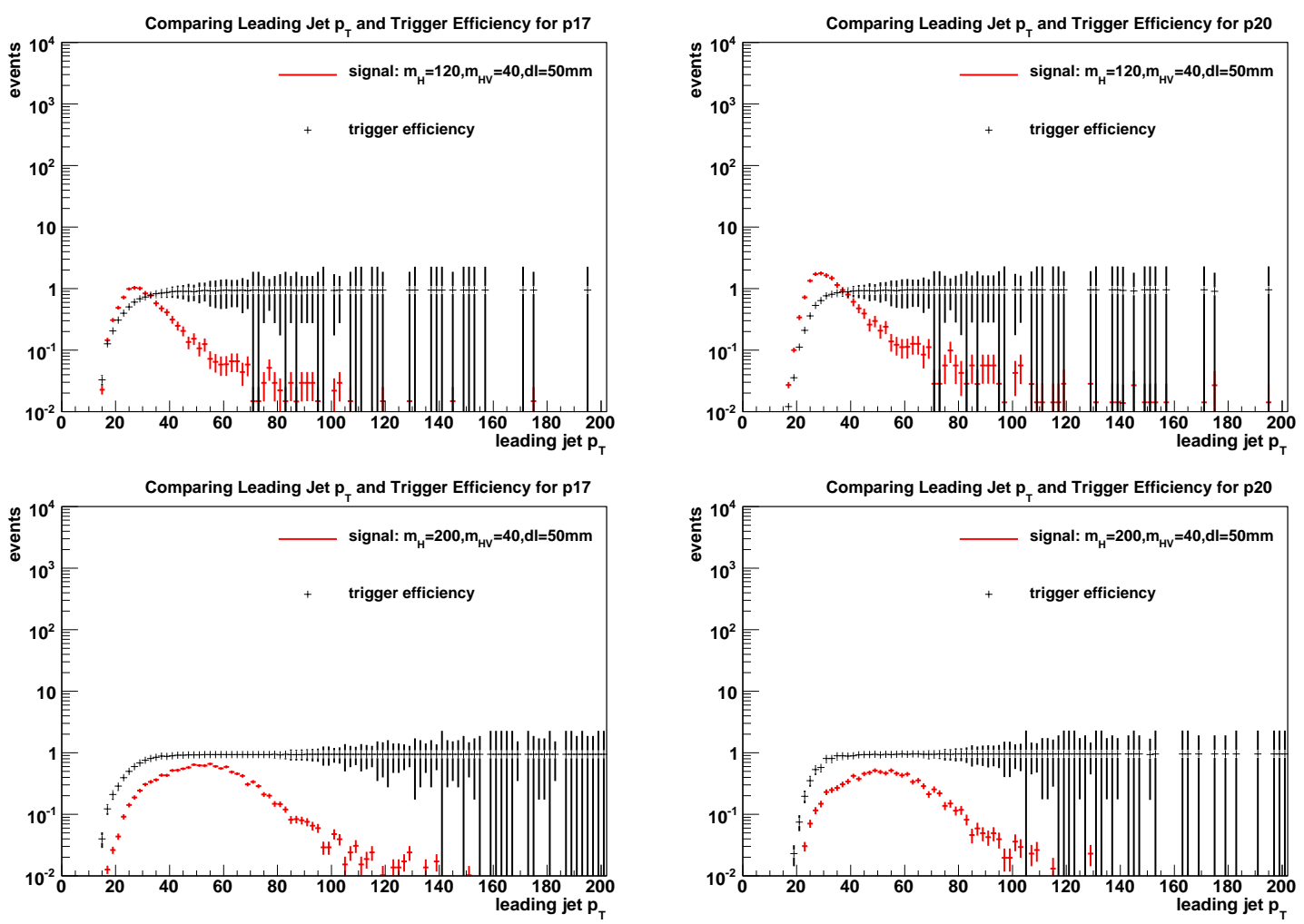

Figure 5.9: Leading jet $p_{T}$ and overall trigger efficiency as a function of leading jet $p_{T}$ for one signal sample. The plot on the left show the total p17 trigger efficiency while the plot on the right show total p20 trigger efficiency.

\begin{tabular}{c|c|c|c|c}
\hline $\begin{array}{c}\text { signal sample } m_{H}(\mathrm{GeV}), m_{H V} \\
(\mathrm{GeV}), \mathrm{dl}(\mathrm{mm})\end{array}$ & $\begin{array}{c}\text { muon } \\
\text { L1 }\end{array}$ & $\begin{array}{c}\text { jet } \\
\text { L1 }\end{array}$ & $\begin{array}{c}\text { muon } \\
\text { L2 }\end{array}$ & $\begin{array}{c}\text { track } \\
\text { L3 }\end{array}$ \\
\hline \hline$m_{H}=90, m_{H V}=15, \mathrm{dl}=50$ & $100 \%$ & $57 \%$ & $100 \%$ & $95 \%$ \\
$m_{H}=90, m_{H V}=40, \mathrm{dl}=50$ & $99 \%$ & $42 \%$ & $100 \%$ & $97 \%$ \\
$m_{H}=120, m_{H V}=15, \mathrm{dl}=25$ & $100 \%$ & $82 \%$ & $100 \%$ & $98 \%$ \\
$m_{H}=120, m_{H V}=15, \mathrm{dl}=50$ & $100 \%$ & $84 \%$ & $100 \%$ & $97 \%$ \\
$m_{H}=120, m_{H V}=15, \mathrm{dl}=100$ & $100 \%$ & $84 \%$ & $100 \%$ & $96 \%$ \\
$m_{H}=120, m_{H V}=40, \mathrm{dl}=50$ & $100 \%$ & $58 \%$ & $100 \%$ & $98 \%$ \\
$m_{H}=200, m_{H V}=15, \mathrm{dl}=50$ & $99 \%$ & $97 \%$ & $100 \%$ & $98 \%$ \\
$m_{H}=200, m_{H V}=40, \mathrm{dl}=50$ & $99 \%$ & $94 \%$ & $100 \%$ & $99 \%$ \\
\hline
\end{tabular}

Table 5.4: The average trigger efficiency of each p17 trigger level for each signal sample. The p17 trigger is MU_2TRK3_L2M0. Events are required to pass the cut0 pre-selection (refer to table 6.1 for the definition of cut0). 


\begin{tabular}{c|c|c}
\hline $\begin{array}{c}\text { signal sample } m_{H}(\mathrm{GeV}), m_{H V} \\
(\mathrm{GeV}), \mathrm{dl}(\mathrm{mm})\end{array}$ & $\begin{array}{c}\text { average } \\
\text { trigger } \\
\text { efficiency }\end{array}$ & $\begin{array}{c}\text { average } \\
\text { uncertainty }\end{array}$ \\
\hline \hline$m_{H}=90, m_{H V}=15, \mathrm{dl}=50$ & $47 \%$ & $14 \%$ \\
$m_{H}=90, m_{H V}=40, \mathrm{dl}=50$ & $34 \%$ & $17 \%$ \\
$m_{H}=120, m_{H V}=15, \mathrm{dl}=25$ & $76 \%$ & $13 \%$ \\
$m_{H}=120, m_{H V}=15, \mathrm{dl}=50$ & $77 \%$ & $13 \%$ \\
$m_{H}=120, m_{H V}=15, \mathrm{dl}=100$ & $79 \%$ & $13 \%$ \\
$m_{H}=120, m_{H V}=40, \mathrm{dl}=50$ & $48 \%$ & $17 \%$ \\
$m_{H}=200, m_{H V}=15, \mathrm{dl}=50$ & $91 \%$ & $13 \%$ \\
$m_{H}=200, m_{H V}=40, \mathrm{dl}=50$ & $87 \%$ & $15 \%$ \\
\hline
\end{tabular}

Table 5.5: Average trigger efficiency for each signal sample including the jet turn on from figure 5.6. Events are required to pass the cut0 pre-selection (refer to table 6.1 for the definition of cut0). 


\section{Chapter 6}

\section{Analysis}

If a neutral long lived particle arising from a "hidden-valley" model of the type proposed in section 2.3 is produced at $\mathrm{D} \varnothing$, it may have a distinctive signature which will need to be isolated from the multitude of events which are recorded at DØ. The precise nature of the signature will depend upon the lifetime and mass of the particle as well as the mass of the Higgs Boson from which it originated. This analysis will study a range of values for these three quantities since none of them are known or predicted by any current model. The number of signal events is expected to be small so an accurate background model will be developed. An optimal set of selection criteria is determined for isolating the signal from the background. Then the systematic uncertainties on the signal acceptance and background model will be studied and finally a limit will be placed on the cross section for the production of a heavy object decaying to v-particles. 


\subsection{Discriminating Handles}

Four variables are used to isolate the signal from the background: SV mass, SV multiplicity, SV collinearity, and number of SVs per event.

The invariant mass is reconstructed from the four-momenta of the outgoing tracks from a SV. Figure 6.2 demonstrates that mass is a more discriminating variable when the difference between $m_{H}$ and $m_{H V}$ is greater. In principle the SV mass should only depend on the mass of the parent particle, $m_{H V}$. However, there may be a difference due to reconstruction efficiency; when the difference between $m_{H}$ and $m_{H V}$ $\left(m_{H}-m_{H V}\right)$ is small, the v-particle is less boosted and its decay products will be emitted at larger angles which have a lower reconstruction efficiency, producing a lower mass measurement.

Secondary-vertex multiplicity refers to the number of tracks which are attached to a given secondary vertex. Signal contains a large number of tracks compared to background as demonstrated in figure 6.3. Similarly to mass, the multiplicity of tracks is a more powerful variable when the difference between $m_{H}$ and $m_{H V}$ is greater corresponding to a more boosted v-particle.

Secondary-vertex collinearity is defined as the cosine of the angle between the vector sum of the momentum of the decay products and the direction the parent. The collinearity is graphically illustrated in figure 6.1. We expect collinearity to be a more discriminating variable when the v-particle is less boosted and its decay products are emitted at larger angles, which is demonstrated in figure 6.3. Collinearity provides

a contrasting discriminant to mass. If all particles were reconstructed $100 \%$ of the time, the collinearity would always be 1 . Some tracks are not reconstructed due to detector or software inefficiencies while others, such as neutrinos, are impossible to 


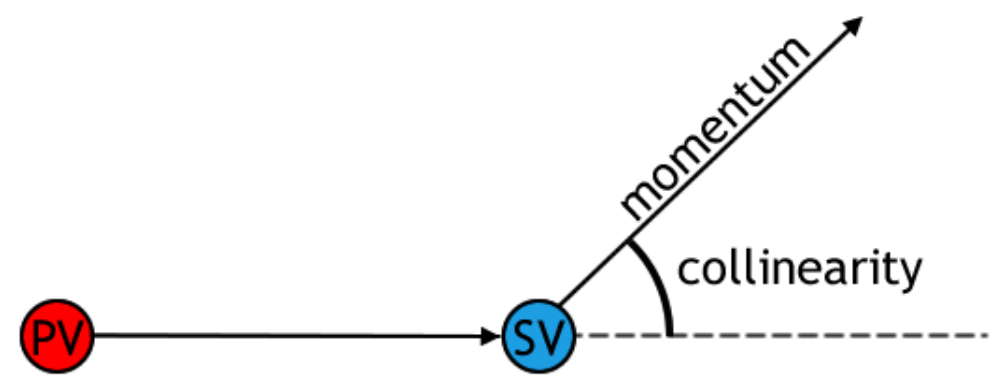

Figure 6.1: The vertex collinearity is defined as the cosine of the angle between the vector sum of the momentum of the decay products and the direction of the parent.

reconstruct because they are electrically neutral and simply do not produce tracks.

Since it is extremely rare for a background process to produce more than one highly-displaced secondary-vertex, requiring two or more SVs increases the fraction of signal content by about two orders of magnitude. This cut reduces the total number of data events by a factor of 1000; it is necessary since the background would otherwise dominate, refer to table 6.3 for the effect of this cut.

\subsection{Event Pre-Selection}

The purpose of pre-selection is twofold: increase the signal significance so that future cuts can be optimized and exclude poorly modeled portions of the data because of limitations in computing resources, we apply certain cuts during simulation and do not expect to model all aspects of data well..

We expect signal events to preferentially produce SVs with a large number of attached tracks, therefore we cut SVs with track multiplicity less than four as in figure 6.16.

Interactions with detector material are a major source of background. In order to 

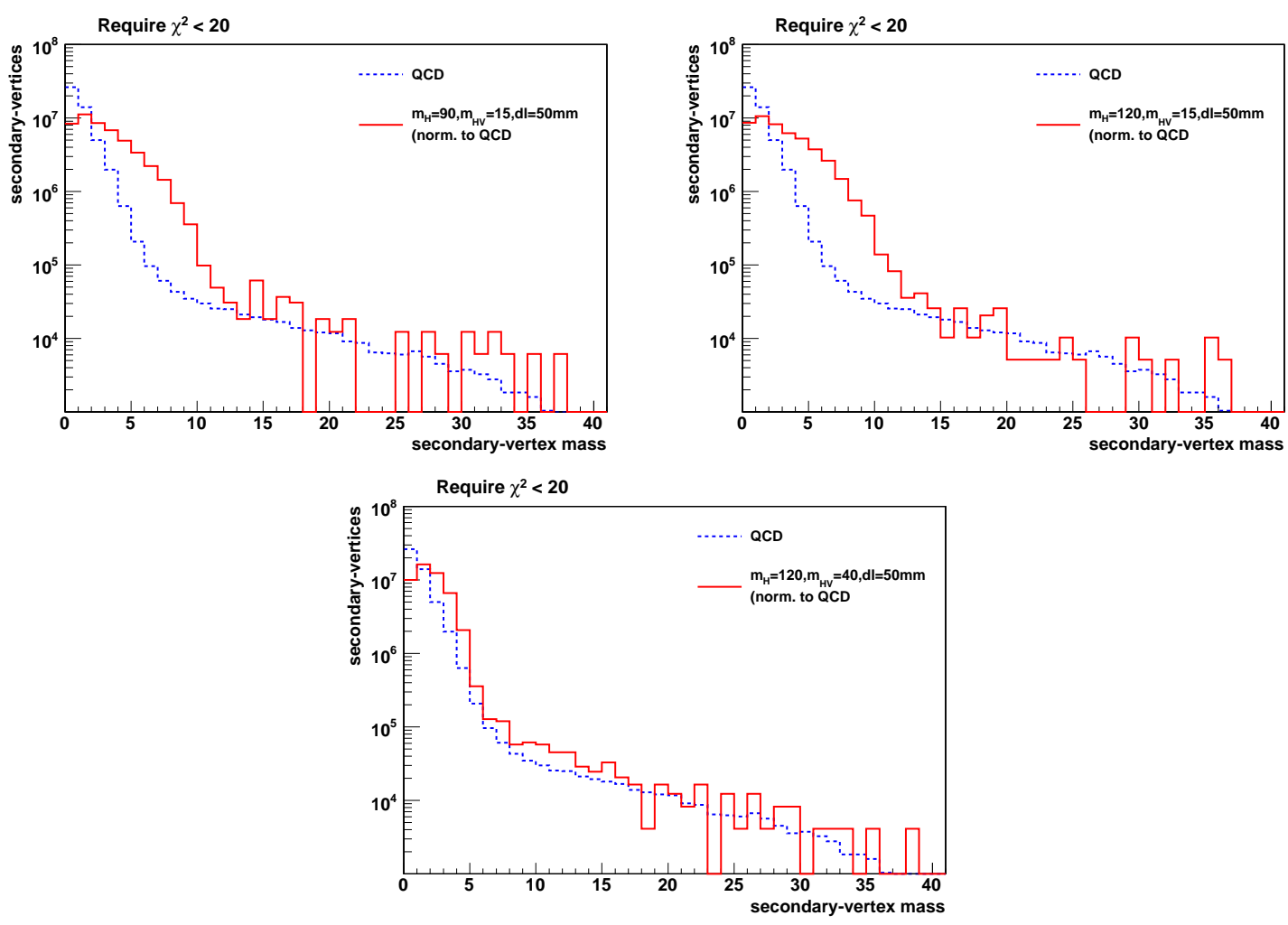

Figure 6.2: The secondary-vertex mass for three different signal hypotheses and background. Signal is normalized to QCD to illustrate the difference in shape. 

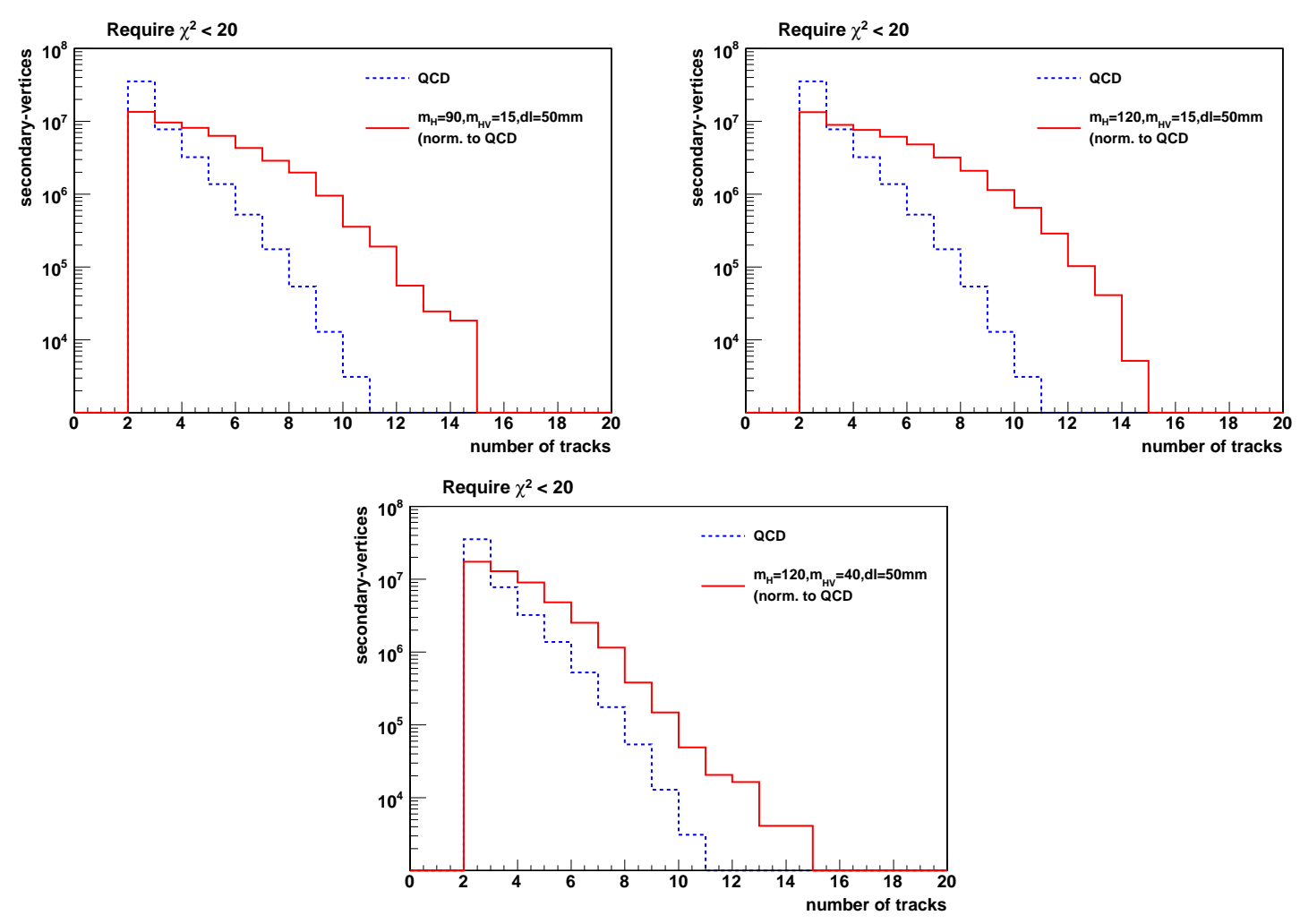

Figure 6.3: The multiplicity of tracks attached to secondary-vertices for three different signal hypotheses and background. Signal is normalized to QCD to illustrate the difference in shape. 

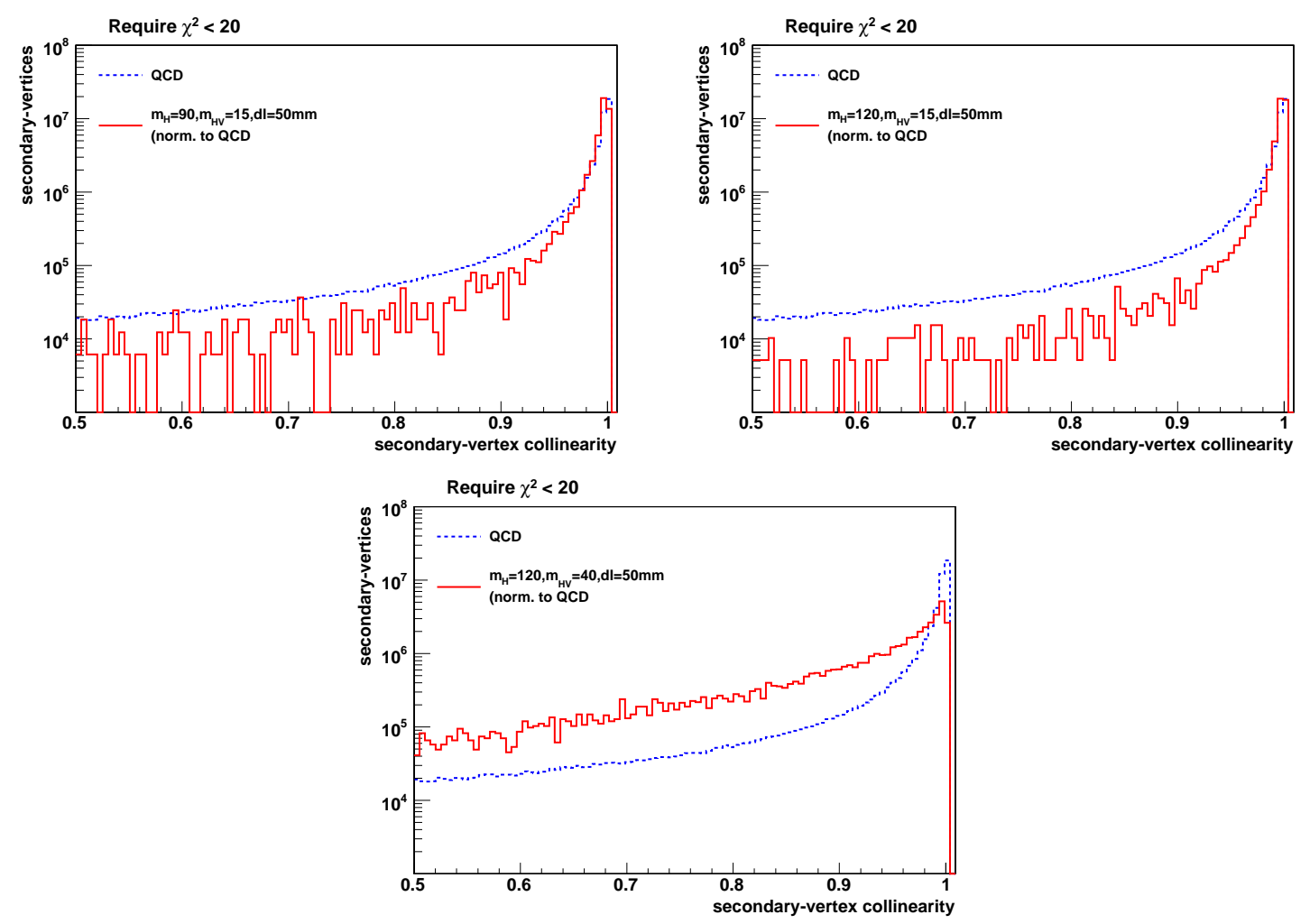

Figure 6.4: The collinearity of tracks attached to secondary-vertices for three different signal hypotheses and background. Signal is normalized to QCD to illustrate the difference in shape. 
quantify the material regions, we construct a map of SVs in the $x y$-plane (figure 6.6) and the $z r$-plane (figure 6.5) of the detector. Secondary-vertices that occur in regions of high density are then cut. High density is found from the density histograms, refer to figure 6.7 for the density in the $x y$-plane and figure 6.8 for the density in the $z r$ plane. The precise location of the density cut is not optimized; it is merely intended to approximately remove the areas of highest density. Appendix $\mathrm{C}$ demonstrates the result of the density cuts on preselected events.

The SV $\chi^{2}$ measures how well a vertex is reconstructed from its constituent tracks, refer to figure 6.10 . While $\chi^{2}$ isn't perfectly modeled, we cut on $\chi^{2}$ to exclude manifestly badly reconstructed vertices. Figure 6.9 shows that the properties of vertices with large $\chi^{2}$ do not agree with the properties of low $\chi^{2}$ vertices. The breaking point for agreement occurs near $\chi^{2}=20$, therefore this cut is added to the pre-selection criteria.

The two dimensional decay length $(\mathrm{dl} 2 \mathrm{~d})$, defined as the decay length in the $x y$ plane, is shown in figure 6.11. The spikes in the plot are due to material interaction in the SMT. We exclude events with $d l 2 d<1.6 \mathrm{~cm}$ to avoid b-decays close to the beamline.

A final data quality cut is imposed on the solid angle between secondary-vertices to exclude single SVs that could be misreconstructed as two or SVs that are extremely close and are chosen to count as one. Figure 6.12 shows the cut requiring all pairs of SVs to have $\Delta R>0.5$ between them, where the angles are between the lines connecting the primary-vertex to the secondary-vertices.

After the pre-selection is performed, the QCD is normalized to the data. The normalization factor is 1.171 for p17 and 0.7460 for p20 (any uncertainty on these 

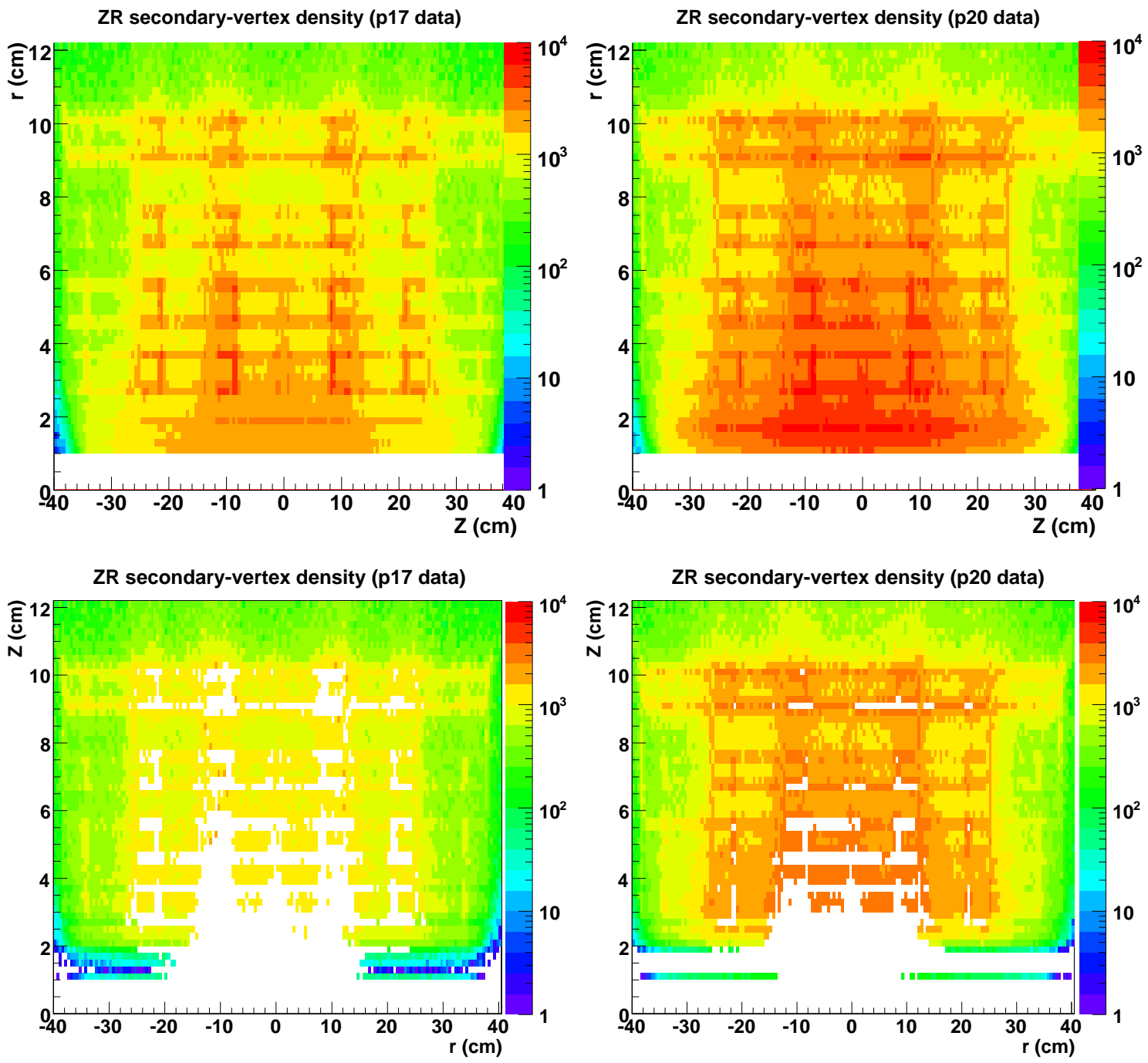

Figure 6.5: Material map before and after cutting out areas of high SV density for the p17 and p20 data sets. Secondary-vertices with track multiplicity $\geq 3$ are used to generate the map. No other pre-selection criteria are required for events in this plot. 

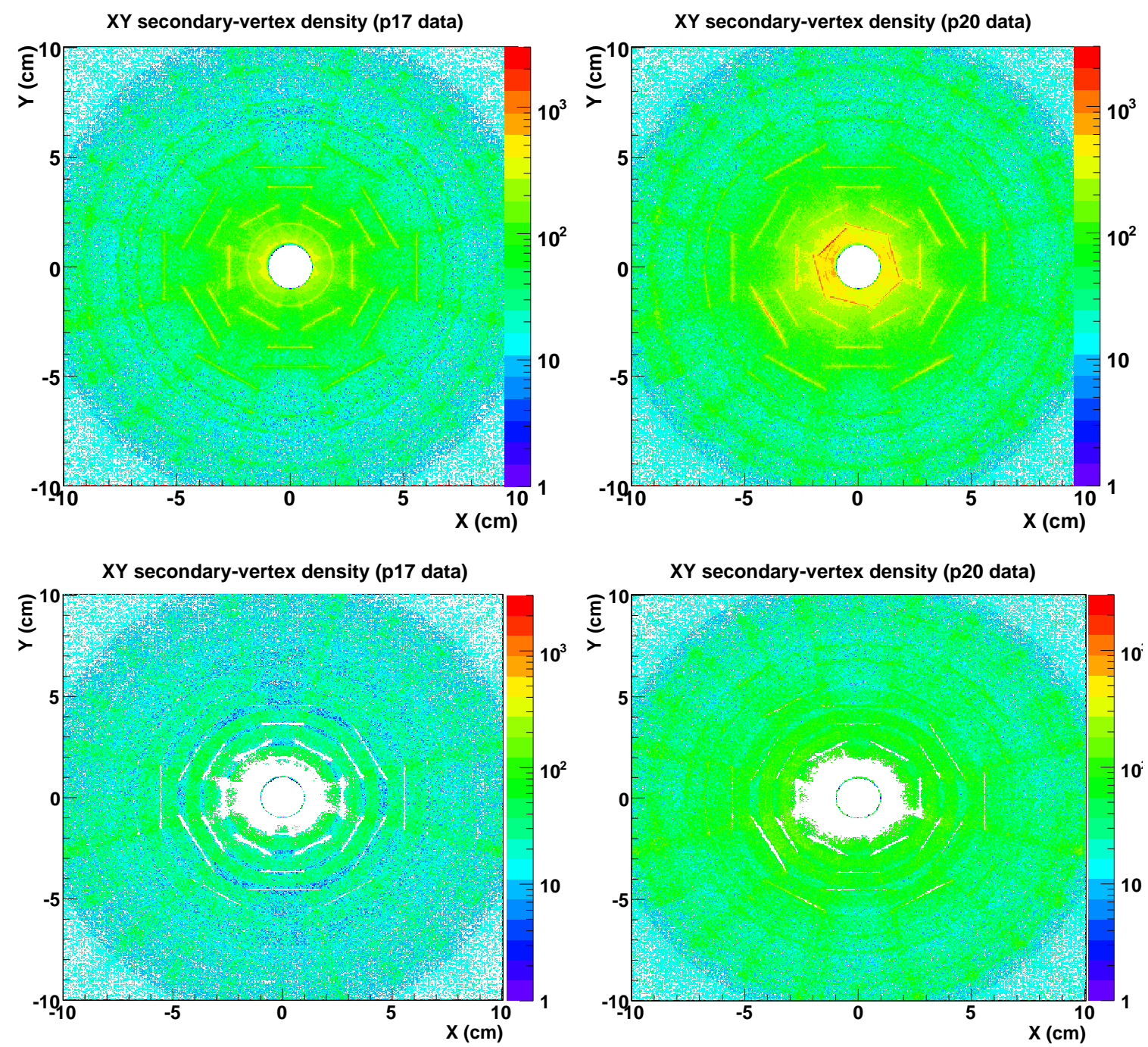

Figure 6.6: Material map of the $x y$-plane before and after cutting out areas of high SV density for the p17 and p20 data sets. Secondary-vertices with track multiplicity $\geq 3$ are used to generate the map. No other pre-selection criteria are required for events in this plot. 

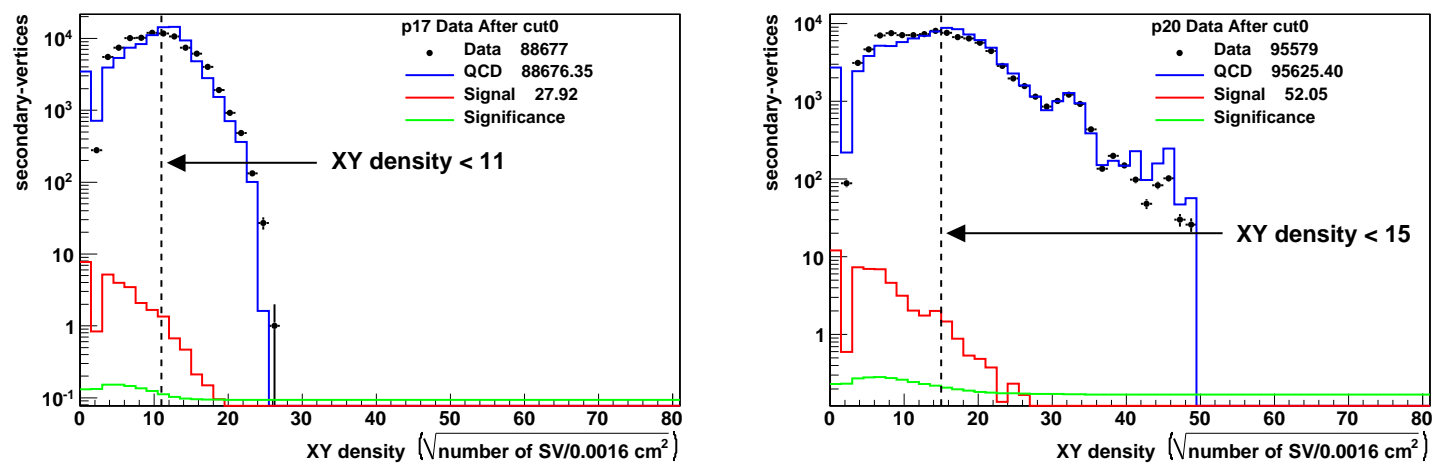

Figure 6.7: The square root of the density of secondary-vertices in the $x y$-plane after the cut0 pre-selection. The dashed line indicates the location of the pre-selection cut.
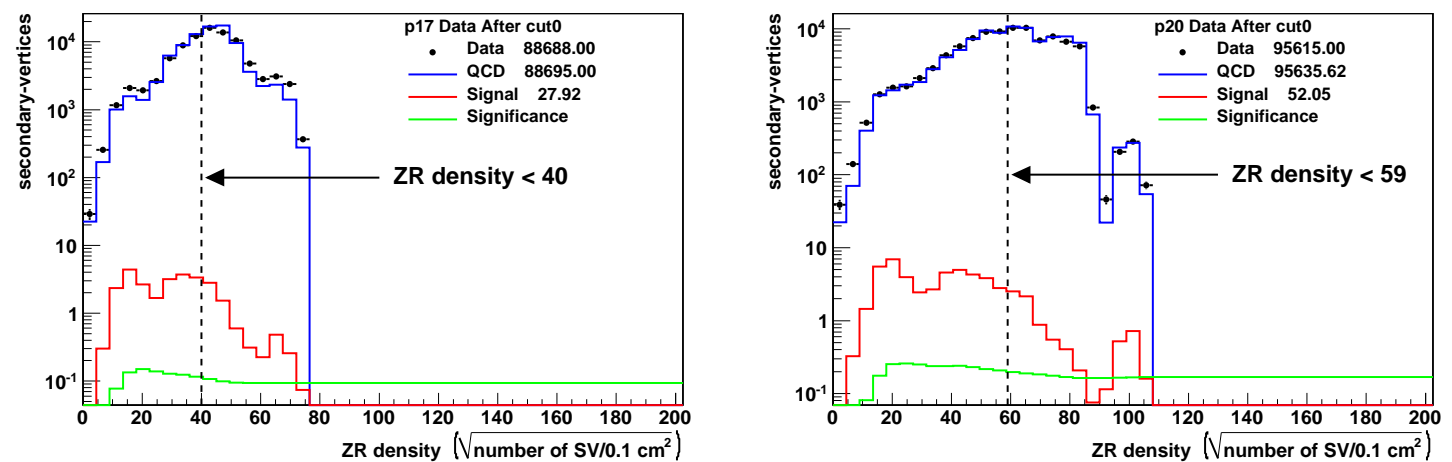

Figure 6.8: The square root of the density of secondary-vertices in the $x y$-plane after the cut0 pre-selection. The dashed line indicates the location of the pre-selection cut. 

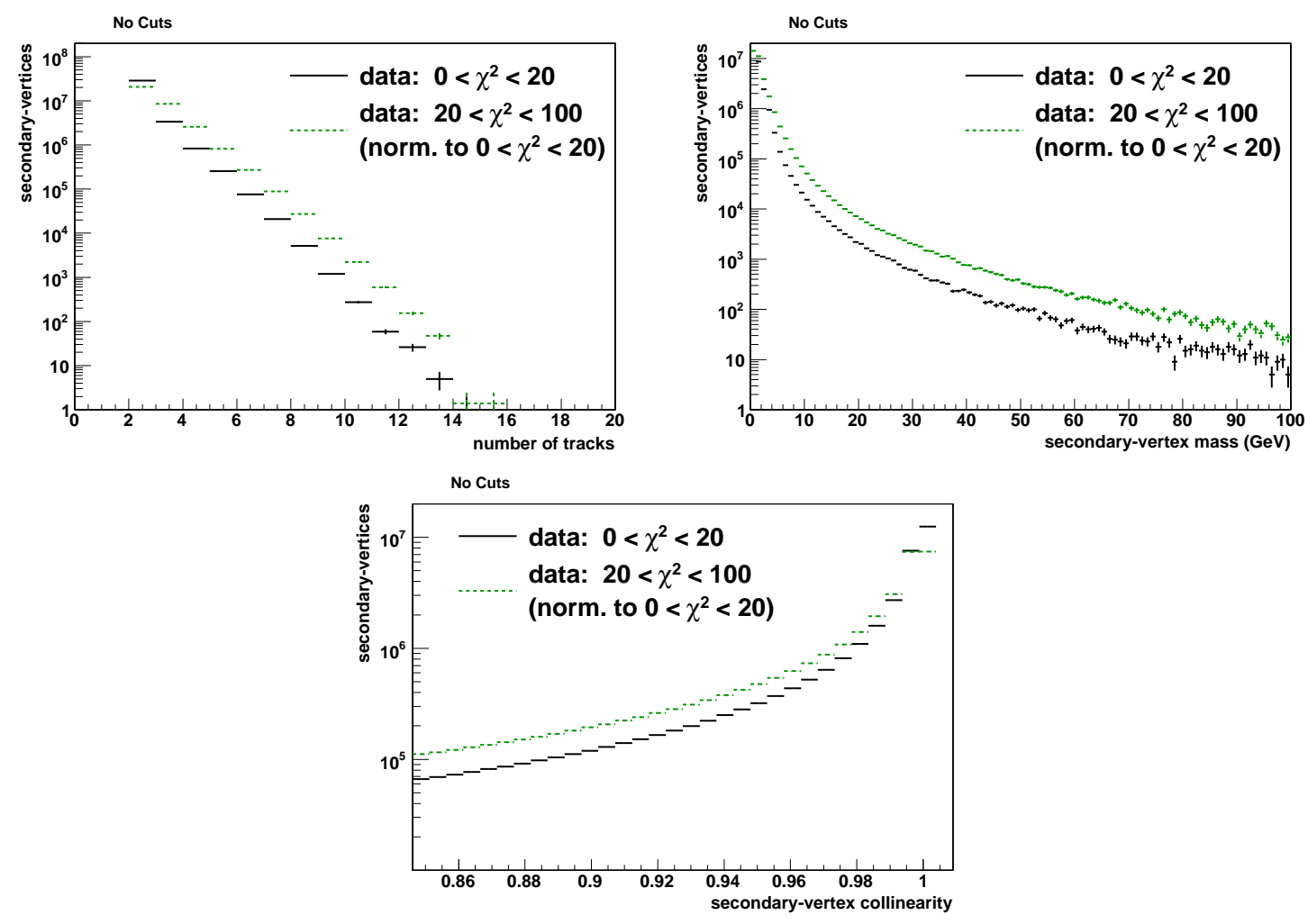

Figure 6.9: Comparison of discriminating variables for data (combined p17 and p20) with $\chi^{2}(S V)<20$ and data with $20<\chi^{2}(S V)<100$. No other cut beyond the $\chi^{2}$ cut is imposed on the events. 


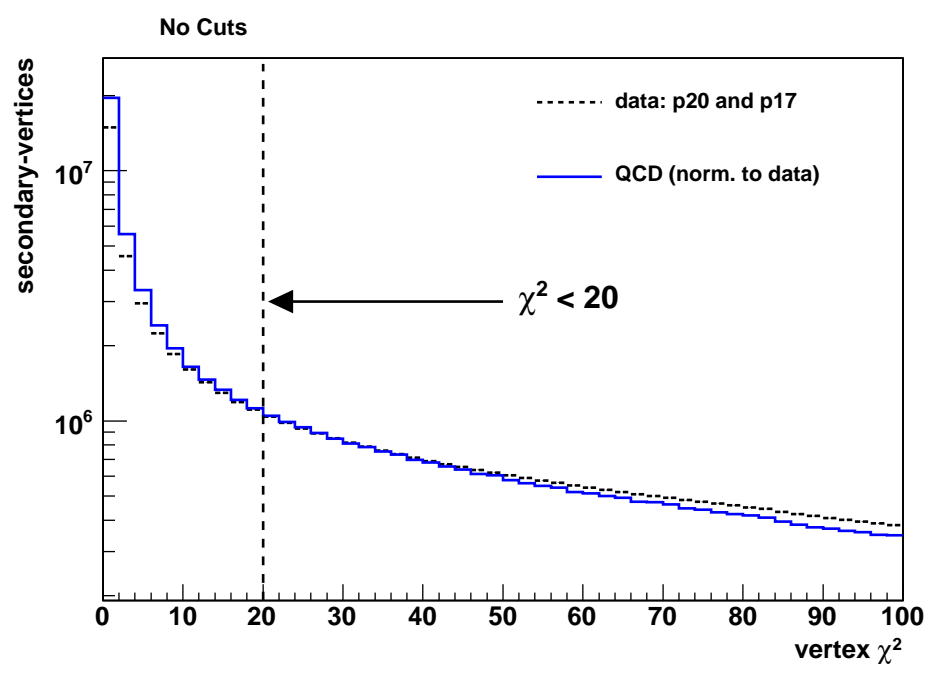

Figure 6.10: Secondary-vertex $\chi^{2}$ per degree of freedom, there were no pre-selection criteria placed on events in this plot. The dashed line at $\chi^{2}=20$ shows the location of the pre-selection cut. Data is combined p17 and p20.

quantities is negligible). Table 6.1 summarizes the pre-selection cuts.

\subsection{Background Modeling}

The quality of the background model is of primary importance so we develop a method of verifying it. We split the data into two distinct sets: the first set contains at least one secondary-vertex per event (1SV) and the second set contains at least two secondary-vertices per event (2SV), refer to figure 6.13. All secondary-vertices are required to pass the pre-selection criteria listed in table 6.1. Since the signal content of the $2 \mathrm{SV}$ set is about $4 \%$ as compared to $.04 \%$ for the $1 \mathrm{SV}$ set, we use the $1 \mathrm{SV}$ set to compare the data and $\mathrm{MC}$ and perform corrections to account for differences in resolution which is slightly better in $\mathrm{MC}$ than in data. We correct for this effect with Gaussian smearing functions which are also applied to signal events. A random 

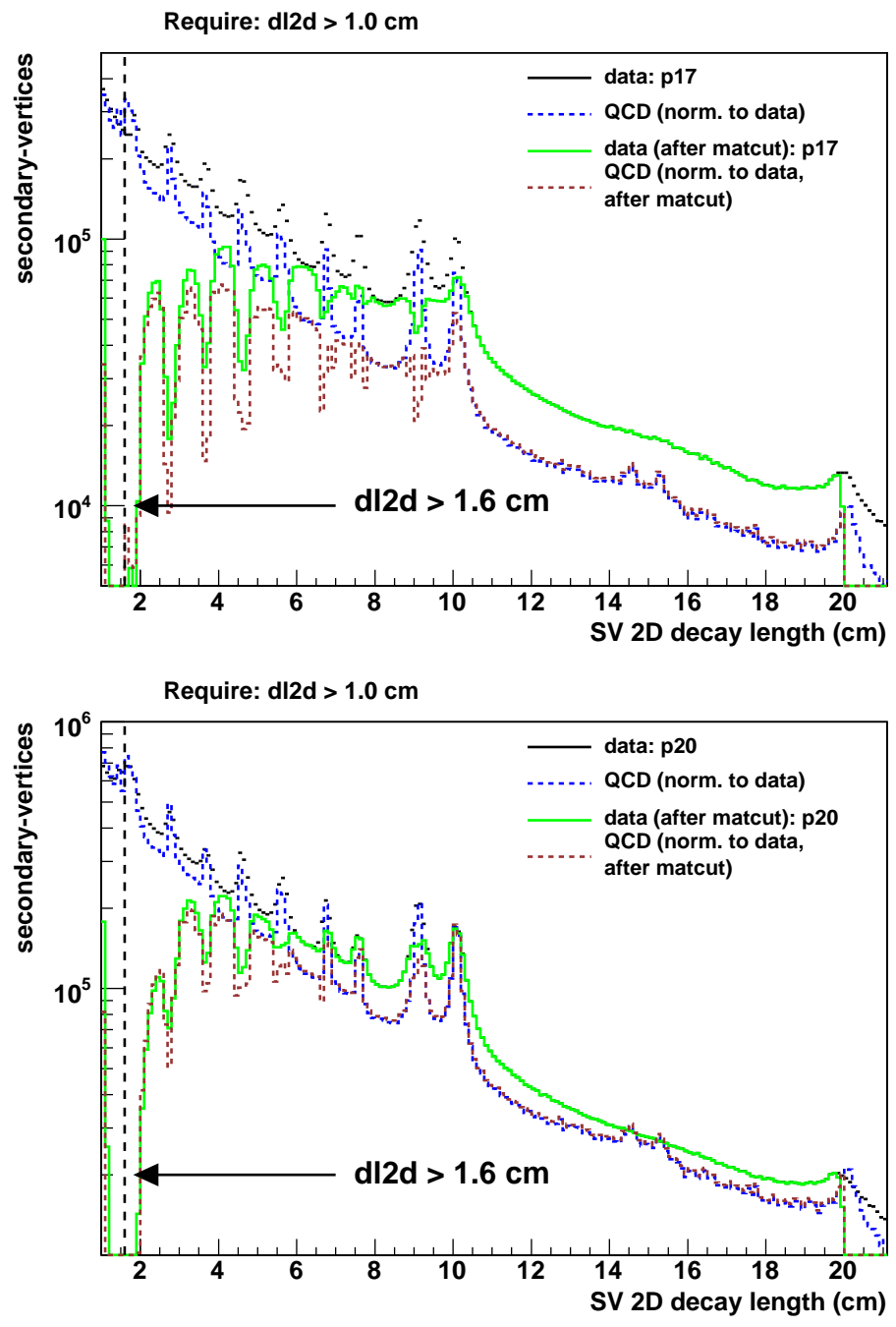

Figure 6.11: Two dimensional decay length. The dashed line at $1.6 \mathrm{~cm}$ shows the location of the pre-selection cut. 

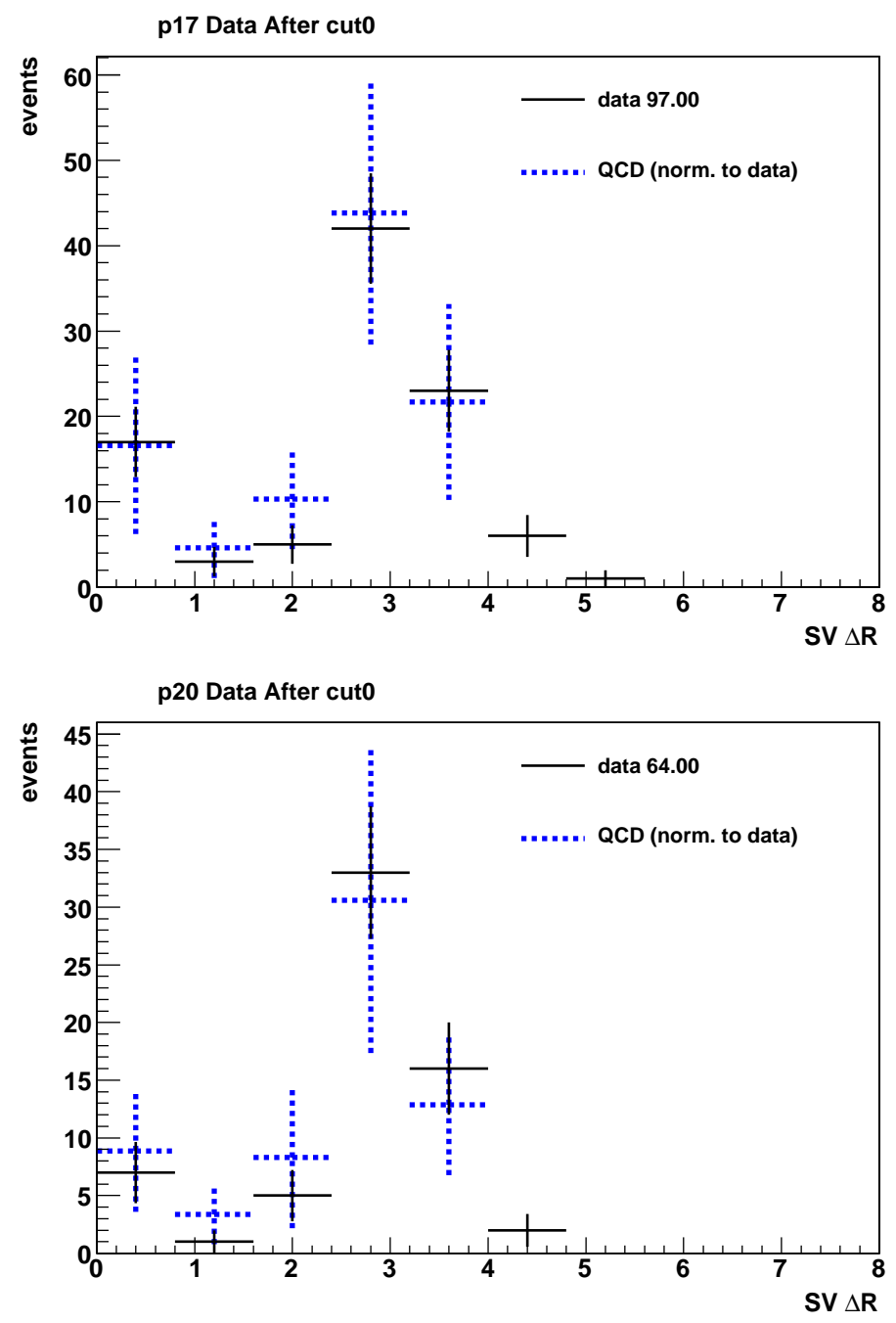

Figure 6.12: $\Delta R$ between secondary-vertices after the cut0 pre-selection requirement for the p17 and p20 data sets. Two SVs per event are required. QCD is normalized to data. 


\begin{tabular}{c|c|c|c}
\hline pre-selection requirement & $\mathrm{p} 17$ & $\mathrm{p} 20$ & label \\
\hline \hline initial number of events & $1.726 \times 10^{7}$ & $3.275 \times 10^{7}$ & nocut \\
\hline SV dl2d $>1.6 \mathrm{~cm}$ & $1.153 \times 10^{7}$ & $2.050 \times 10^{7}$ & \\
SV $\chi^{2}<20$ & $7.574 \times 10^{6}$ & $1.254 \times 10^{7}$ & cut0 \\
SV multiplicity $>4$ & $8.860 \times 10^{4}$ & $9.559 \times 10^{4}$ & \\
\hline SV $x y$ material density & $4.935 \times 10^{4}$ & $5.187 \times 10^{4}$ & mat_cut \\
SV $z r$ material density & $2.436 \times 10^{4}$ & $3.560 \times 10^{4}$ & \\
\hline$\Delta R<0.5$ & & & dr_cut \\
\hline
\end{tabular}

Table 6.1: Summary of all pre-selection cuts. The "p17" and "p20" columns indicate the number of data events passing each consecutive cut. Each event must contain at least one SV per event which passes the given pre-selection requirement as well as all of the previous requirements in the table. The $x y$ material density cut requires that events must have at least one SV with $\sqrt{\text { xydensity }}<11$ for p17 and $\sqrt{\text { xydensity }}<$ 15 for p20. The $z r$ material density cut requires that events must have at least one $\mathrm{SV}$ with $\sqrt{z r \text { density }}<40$ for $\mathrm{p} 17$ and $\sqrt{z r \text { density }}<59$ for $\mathrm{p} 20$. The $\Delta R$ cut only applies when there are two or more secondary-vertices in an event.

number generator samples from a Gaussian and applies this to the mass or collinearity. The mass is smeared by a Gaussian with a sigma of $12 \mathrm{GeV}$ which is applied to $0.50 \%$ $(1.25 \%)$ of the events in the p17 (p20) sample. The collinearity is smeared by a Gaussian with a sigma of 0.10 , applied to $1.50 \%$ (1.75\%) of the events in the p17 (p20) sample. The reason for smearing only a fraction of the events is to introduce a tail in the data which is not correctly reproduced by the Monte-Carlo. The width of the Gaussians and the number of $\mathrm{QCD}$ events to which is is applied are found by performing a $\chi^{2}$ fit of the QCD to the data. The logarithm of the number of events in each bin is used because it will tend to favor the tails of the distributions. Figures 6.14 and 6.15 compare the corrected and uncorrected QCD to data.

As expected, track reconstruction efficiency is higher in $\mathrm{MC}$ than in data which is reflected in the data to QCD agreement for track multiplicity shown in figure 6.16. However, multiplicity is not a variable on which we place a tight cut and the effect of 

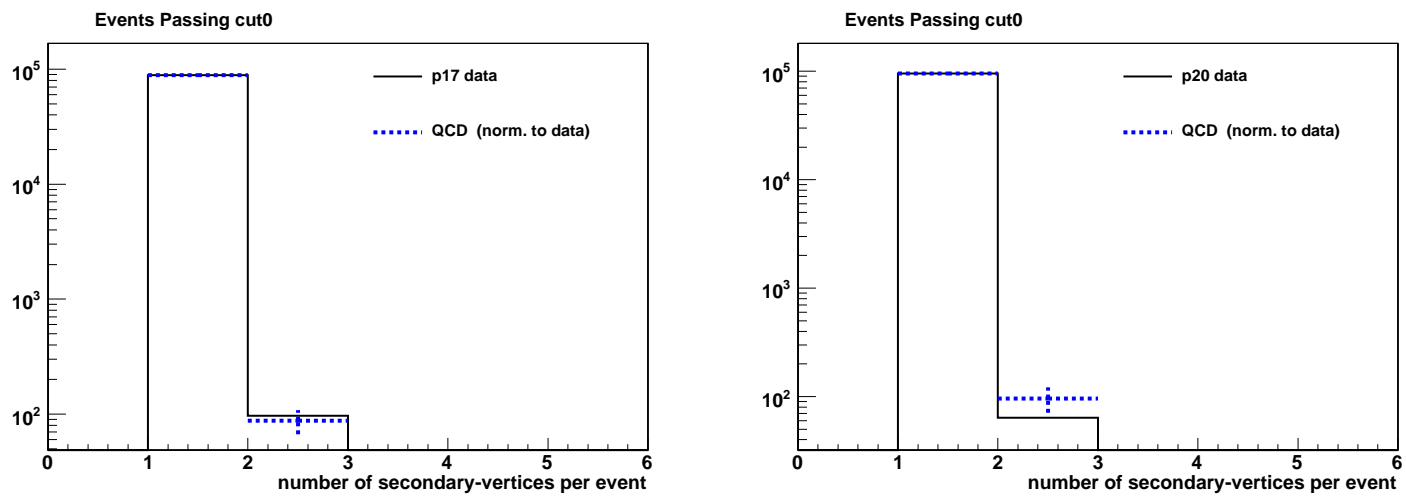

Figure 6.13: The number of secondary-vertices per event for p17 and p20 secondaryvertices passing cut0.

re-weighting the QCD multiplicity to match that of the data's has a small effect on the final results, which we include as a systematic uncertainty (refer to section 6.6.1).

\subsection{Cuts}

After requiring two secondary-vertices, the QCD sample contains very few events which produces large statistical uncertainties. For this reason, we use the smoothing algorithm provided by root, TH1::Smooth() [48], to reduce statistical fluctuations. Figure 6.17 demonstrates the result of smoothing. Smoothing does not alter the final result or the location of the optimized cuts, it merely removes statistical fluctuations that might produce undesirable artifacts.

For the purpose of optimizing the final cut, we only look at the signal and background MC. For the low mass hidden-valley $\left(m_{H V}=15 \mathrm{GeV}\right)$ signal hypotheses the optimal cut is on the SV mass. We can increase the significance of the signal by making a cut on the minimum of the mass of the two secondary-vertices, which is shown in figures $6.18,6.19,6.20,6.21$ and 6.22 . For the high mass signal hypotheses 

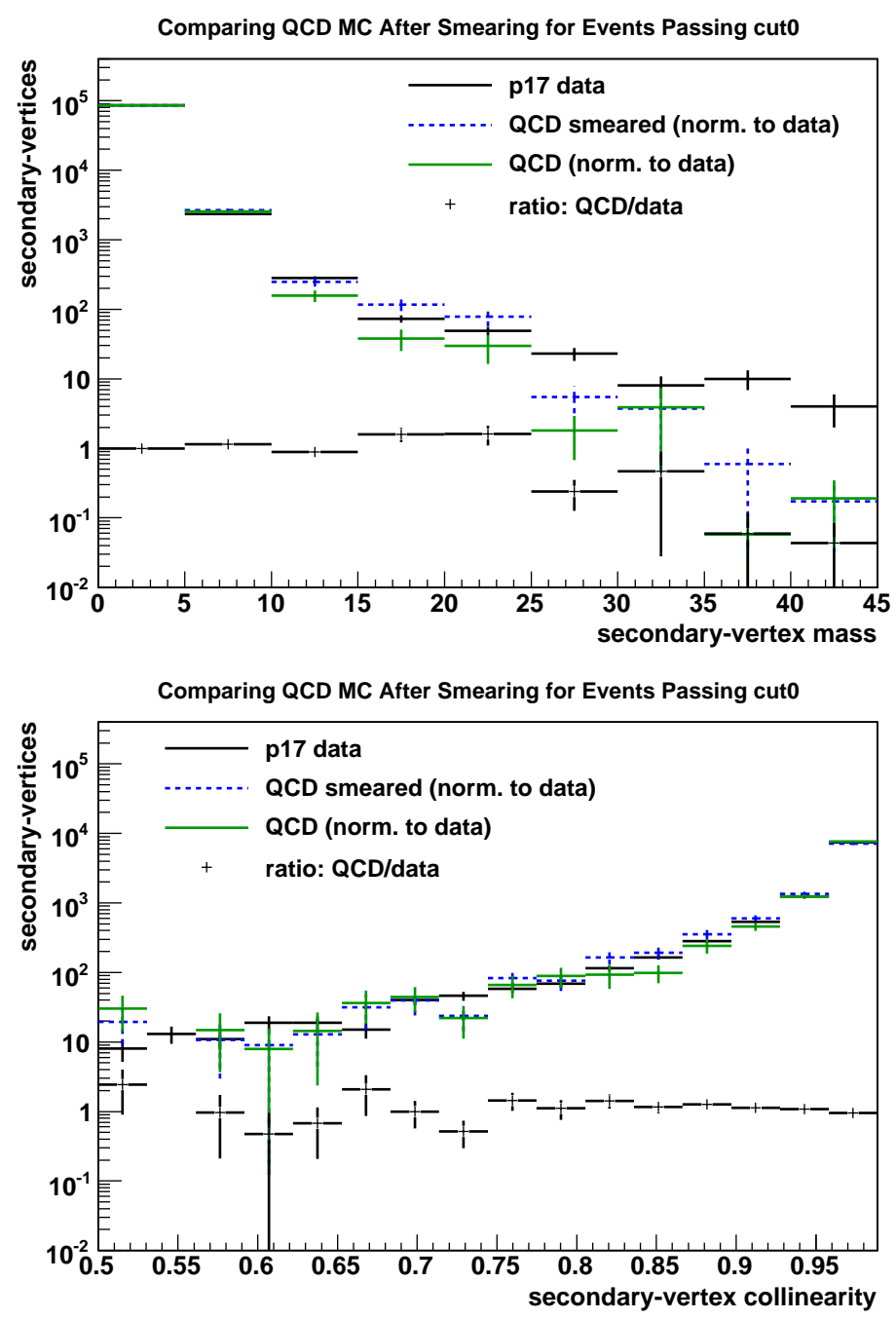

Figure 6.14: Comparing smeared and unsmeared QCD to run 2a (p17) data for SV mass and collinearity. The mass is smeared by a Gaussian with a sigma of $12 \mathrm{GeV}$ which is applied to $0.50 \%$ of the events. The collinearity is smeared by a Gaussian with a sigma of 0.10 , applied to $1.50 \%$ of the events. 

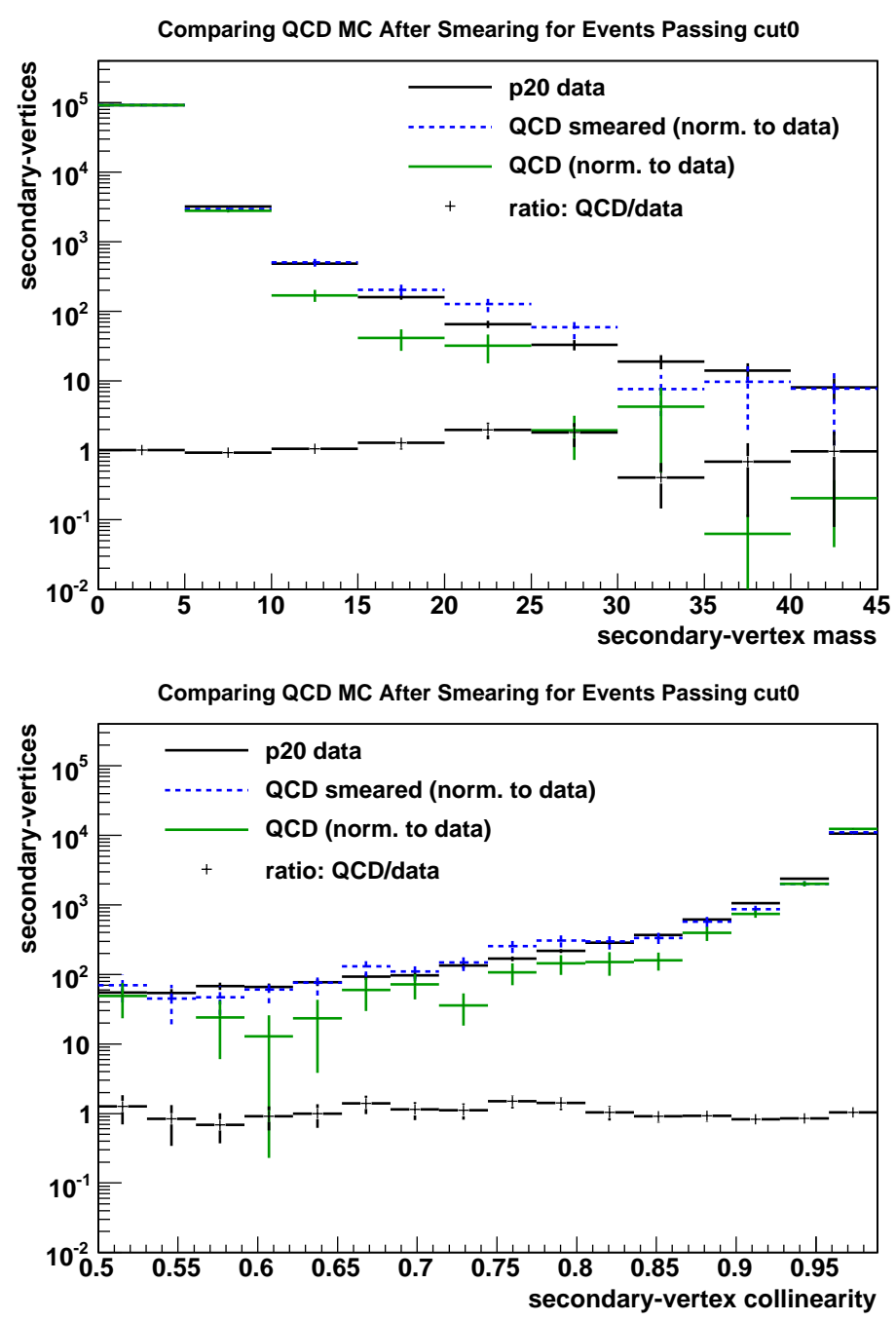

Figure 6.15: Comparing smeared and unsmeared QCD to run 2b (p20) data for SV mass and collinearity. The mass is smeared by a Gaussian with a sigma of $12 \mathrm{GeV}$ which is applied to $1.25 \%$ of the events. The collinearity is smeared by a Gaussian with a sigma of 0.20 , applied to $1.75 \%$ of the events. 

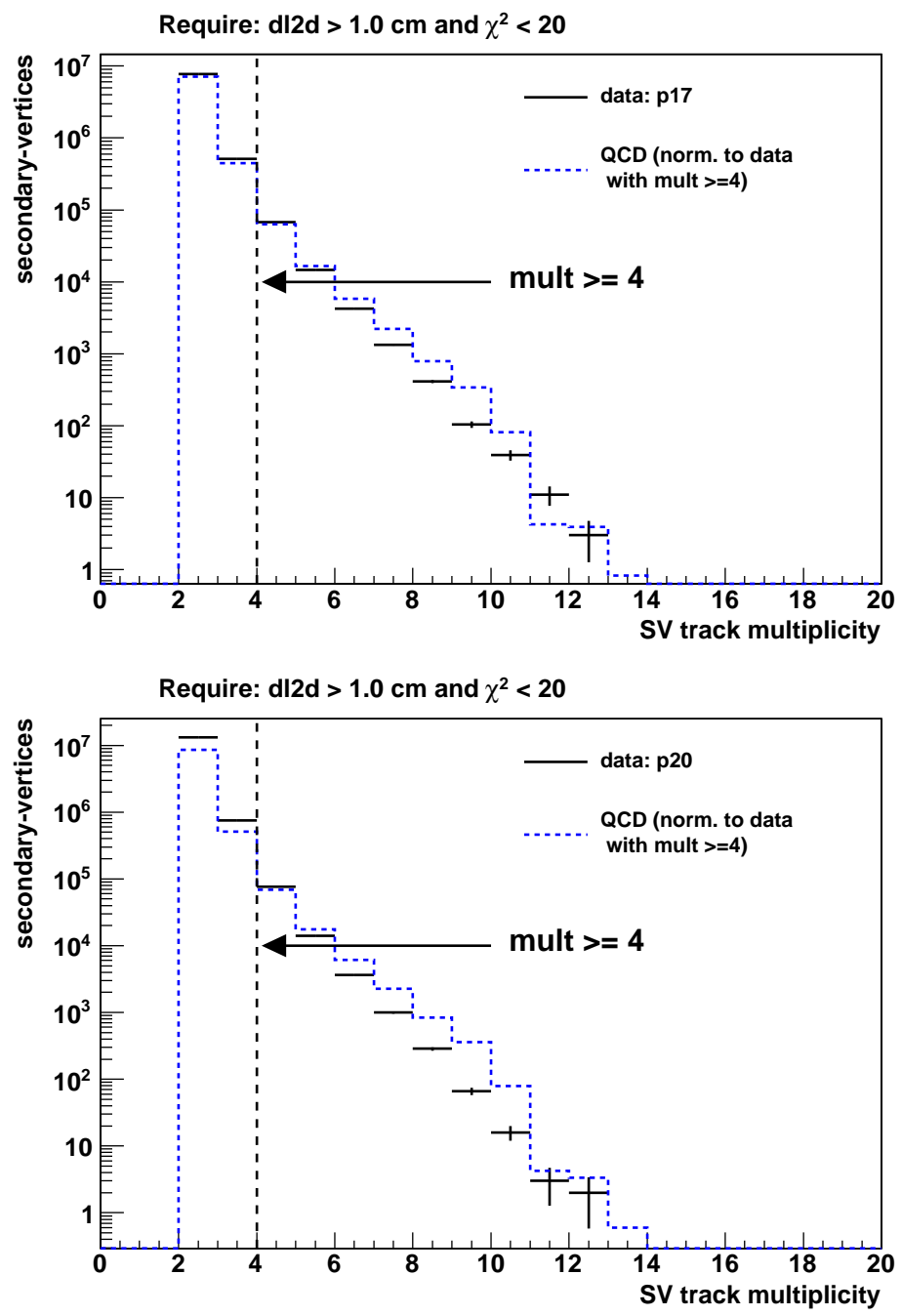

Figure 6.16: Secondary-vertex track multiplicity for p17 and p20 data and MC. SVs in this plot were required to have $\chi^{2}<20$ and $\mathrm{dl} 2 \mathrm{~d}>1.6 \mathrm{~cm} \mathrm{~cm}$ as discussed in section 6.2. QCD was normalized to data for multiplicities $>=4$. 

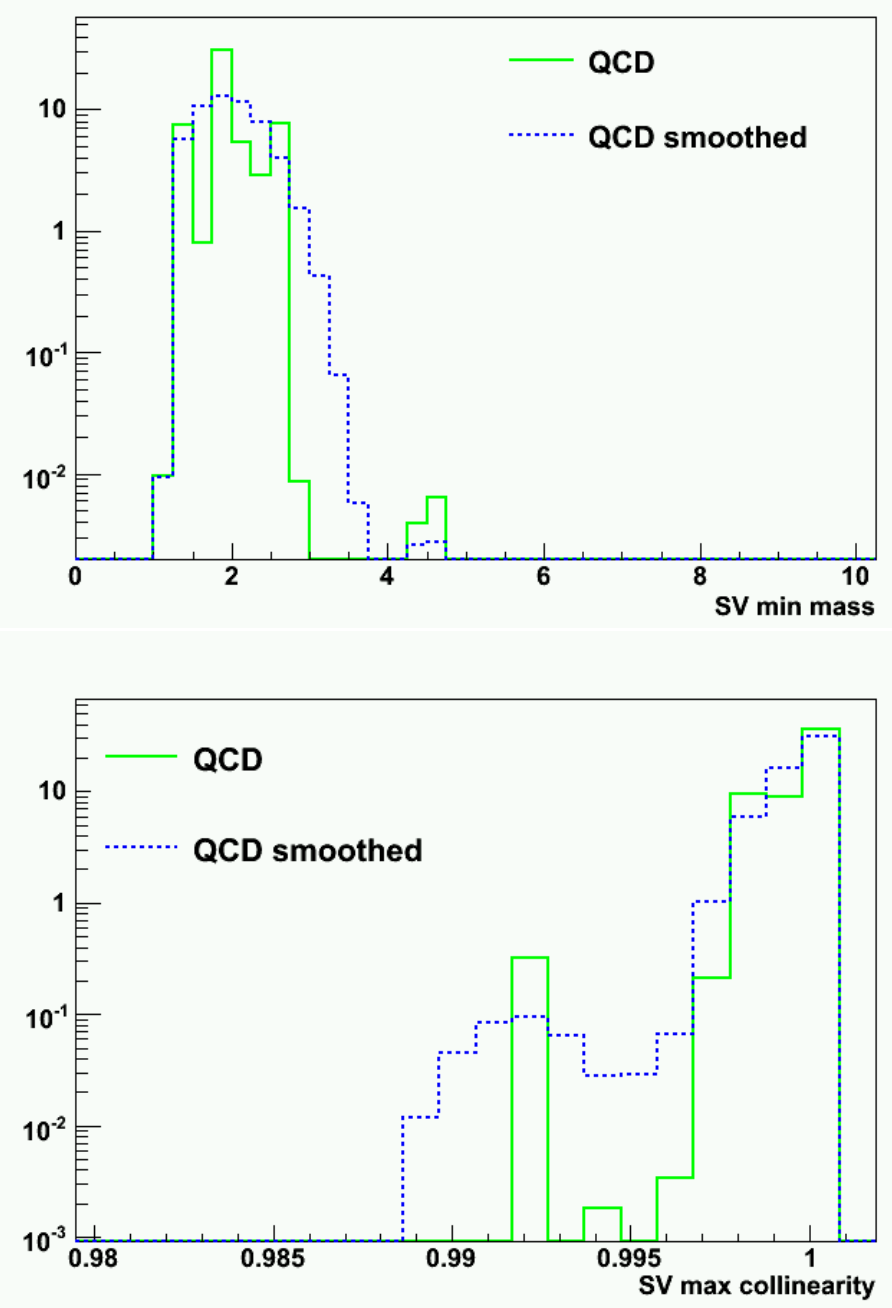

Figure 6.17: An example of the result of smoothing mass and collinearity with the Root function, TH1::Smooth(). 

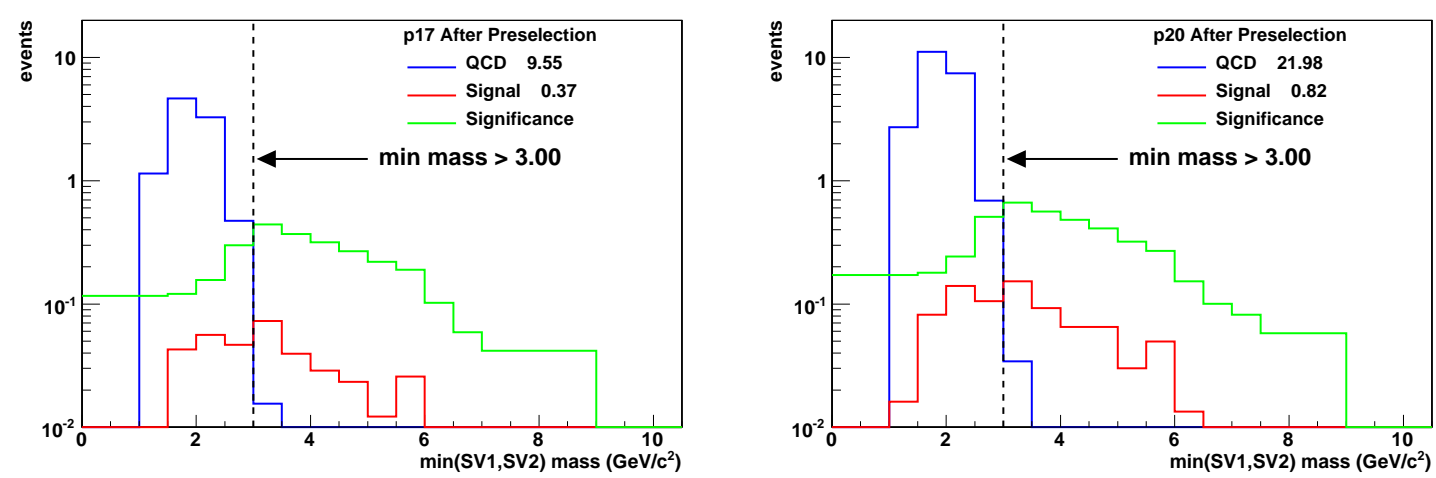

Figure 6.18: The minimum of the mass of the two secondary-vertices. This plot shows the significance of making a cut on the signal $\left(m_{H}=200 \mathrm{GeV}, m_{H V}=15 \mathrm{GeV}, d l=\right.$ $50 \mathrm{~mm}$ ) and background MC.

$\left(m_{H V}=40 \mathrm{GeV}\right)$ the optimal cut is on the SV collinearity. Again, we are able to maximize the significance by cutting on the maximum of the collinearity of the two SVs, refer to figure $6.23,6.24$ and 6.25 .

\subsection{Limit Setting}

The purpose of using statistical techniques is to effectively estimate the value of a parameter given a finite sample of data. In evaluating the validity of a physical model, one generally expects a certain number of events due to known processes (background) and the process that is being evaluated (signal). The average number of observed events is,

$$
\mu=b+\int \mathcal{L} \epsilon \sigma
$$

where $\mathrm{b}$ is the expected number of background events, $\int \mathcal{L}$ is the integrated luminosity, $\epsilon$ is the signal efficiency and $\sigma$ is the signal production cross-section which is the parameter being estimated. The probability of observing $d$ events given mean $\mu$ 

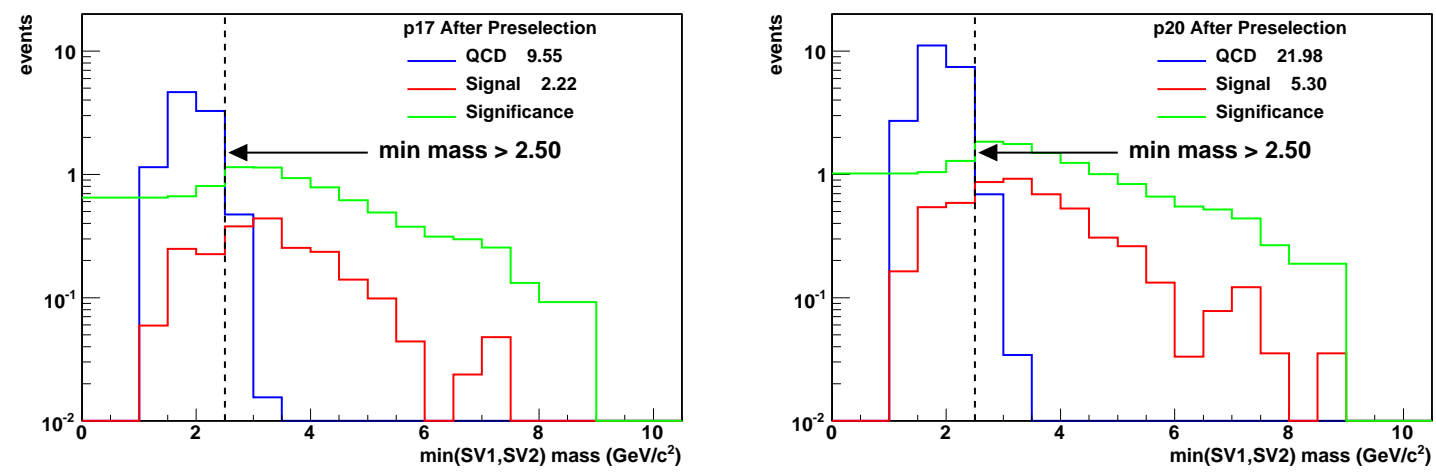

Figure 6.19: The minimum of the mass of the two secondary-vertices. This plot shows the significance of making a cut on the signal $\left(m_{H}=120 \mathrm{GeV}, m_{H V}=15 \mathrm{GeV}, d l=\right.$ $25 \mathrm{~mm}$ ) and background MC.
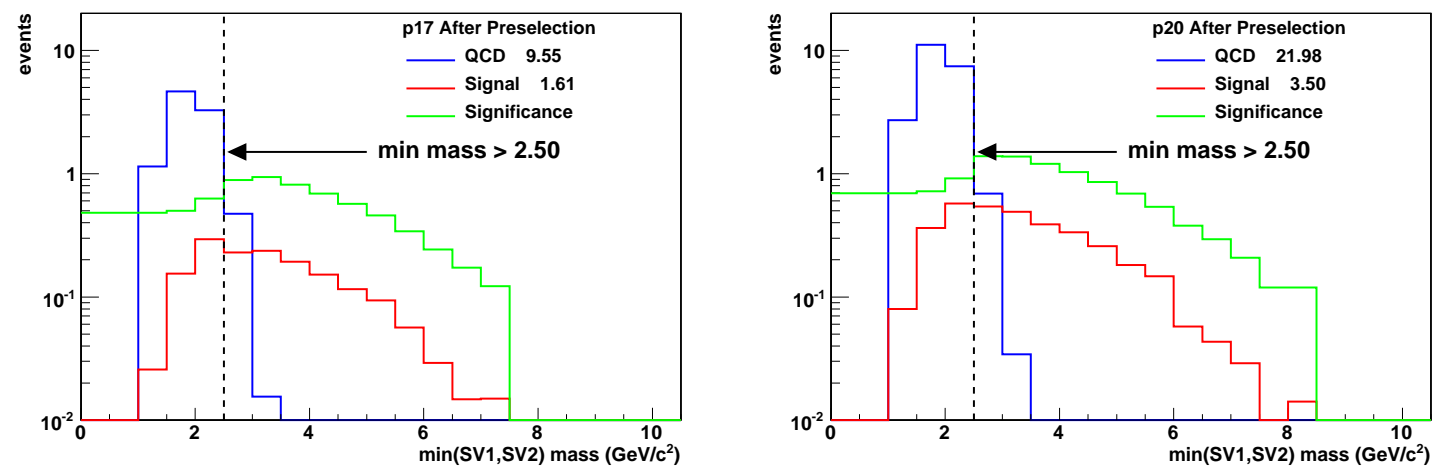

Figure 6.20: The minimum of the mass of the two secondary-vertices. This plot shows the significance of making a cut on the signal $\left(m_{H}=120 \mathrm{GeV}, m_{H V}=15 \mathrm{GeV}, d l=\right.$ $50 \mathrm{~mm}$ ) and background MC. 

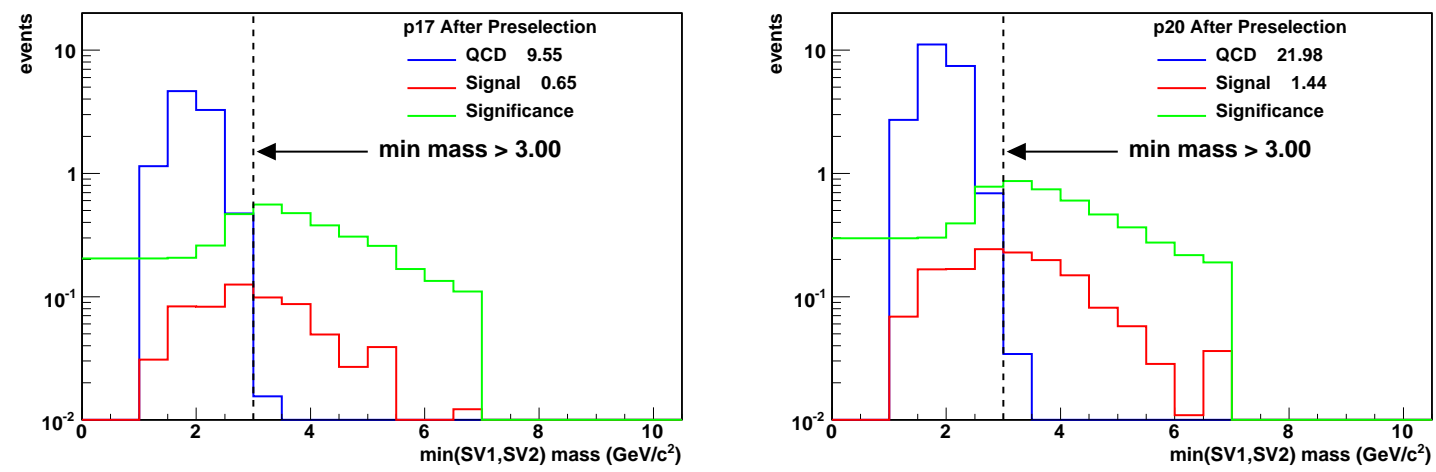

Figure 6.21: The minimum of the mass of the two secondary-vertices. This plot shows the significance of making a cut on the signal $\left(m_{H}=120 \mathrm{GeV}, m_{H V}=15 \mathrm{GeV}, d l=\right.$ $100 \mathrm{~mm}$ ) and background MC.
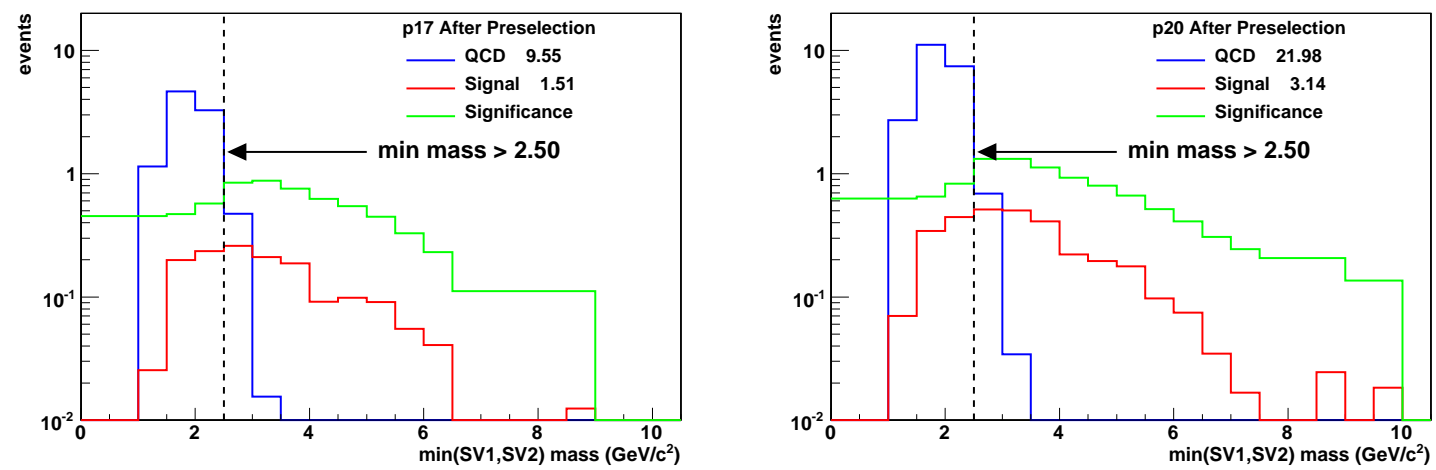

Figure 6.22: The minimum of the mass of the two secondary-vertices. This plot shows the significance of making a cut on the signal $\left(m_{H}=90 \mathrm{GeV}, m_{H V}=15 \mathrm{GeV}, d l=\right.$ $50 \mathrm{~mm}$ ) and background MC. 

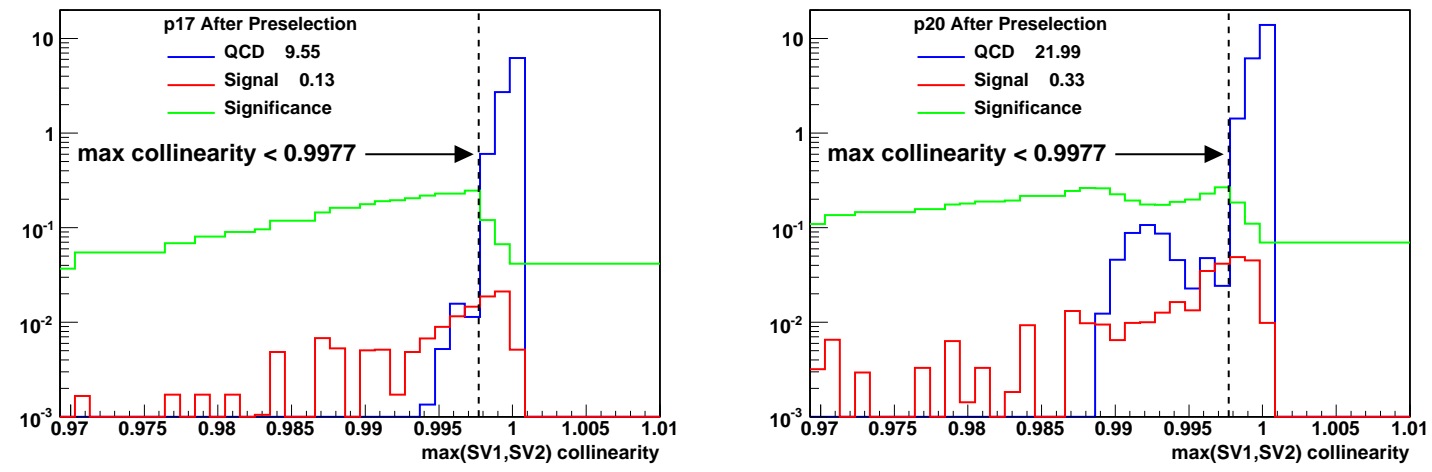

Figure 6.23: The maximum of the collinearity of the two secondary-vertices. This plot shows the significance of making a cut on the signal $\left(m_{H}=200 \mathrm{GeV}, m_{H V}=\right.$ $40 \mathrm{GeV}, d l=50 \mathrm{~mm}$ ) and background MC.
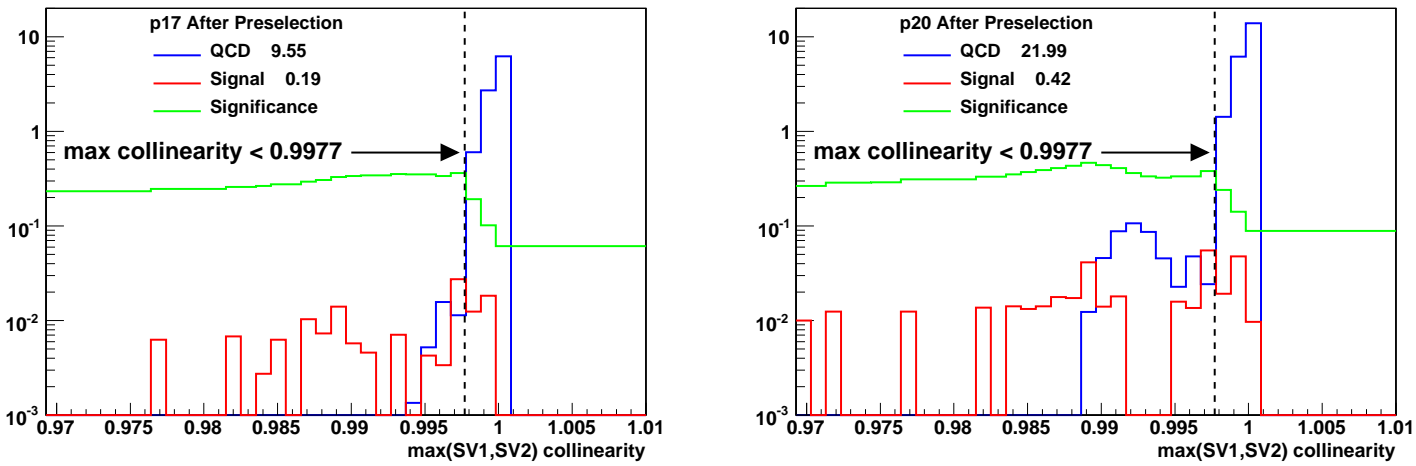

Figure 6.24: The maximum of the collinearity of the two secondary-vertices. This plot shows the significance of making a cut on the signal $\left(m_{H}=120 \mathrm{GeV}, m_{H V}=\right.$ $40 \mathrm{GeV}, d l=50 \mathrm{~mm}$ ) and background MC. 

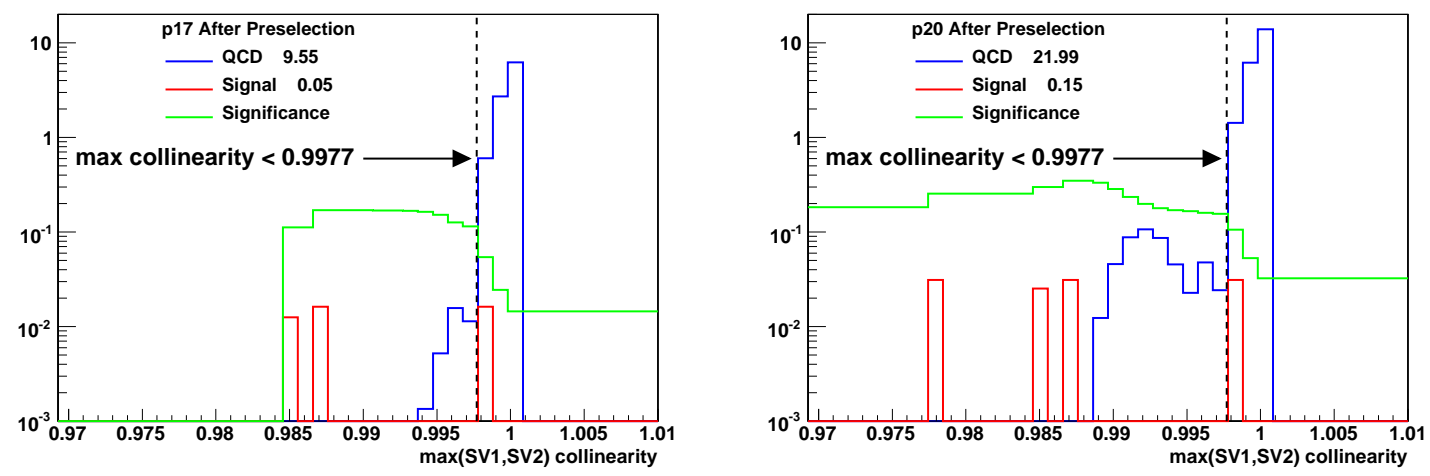

Figure 6.25: The maximum of the collinearity of the two secondary-vertices. This plot shows the significance of making a cut on the signal $\left(m_{H}=90 \mathrm{GeV}, m_{H V}=\right.$ $40 \mathrm{GeV}, d l=50 \mathrm{~mm}$ ) and background $\mathrm{MC}$.

follows Poisson statistics,

$$
P(d \mid \mu)=\frac{e^{-\mu} \mu^{d}}{d !}
$$

The total number of data and predicted background events after all cuts is counted for each trial signal distribution (ie. each combination of $m_{H}, m_{H V}$ and decay length) which is known as a counting experiment. In the absence of a statistically significant number of data events above the predicted background, upper limits are set on the possible production cross section; if the Higgs boson decays to $b$-jets through a hiddenvalley sector, then we are able to set cross-section times branching ratio limits above which the decay could not occur or else it would have been observed as a significant excess. The quantity that determines a statistically significant excess is the p-value; the probability for the background model to reproduce the observed number of events. A small p-value indicates that it is unlikely for the background model to fluctuate to the data in which case the analysis would present evidence or an observation of the proposed signal process. 
The cross-section limit is expressed at a certain confidence-level (C.L.) which indicates the likelihood of obtaining the same result if the experiment is repeated. The precise definition of a confidence-level depends on the approach used for setting the limit and will be discussed further in section 6.5.1. The upper limit on the production cross-section, $\sigma_{U L}$, maximizes the inequality,

$$
\text { C.L. }>\int_{0}^{\sigma_{U L}} d \sigma \rho(\sigma \mid k)
$$

where $\rho(\sigma \mid k)$ is the probability density function (p.d.f.) which is found in section 6.5.2. This analysis uses the modified frequentist, or $C L_{S}$ approach, see $[49,50,51]$. The alternative Bayesian technique [52] was also employed as a cross check and gave similar results.

\subsubsection{The $C L_{S}$ Approach}

The analysis of a search can be formulated in terms of testing a hypothesis; the null hypothesis indicates that the observed data is composed only of background-like events $(b)$ and the alternative indicates it is composed of background and signal-like events $(s+b)$. If we define a test statistic, $Q$, which discriminates signal-like outcomes from background-like ones, the confidence-level for the $s+b$ hypothesis is equal to the probability that the test statistic is less than or equal to the value observed in the experiment, $Q_{o b s}$ :

$$
C L_{s+b}=P_{s+b}\left(Q \leq Q_{o b s}\right)
$$


where $s$ is the number of signal events given by,

$$
s=\int \mathcal{L} \epsilon \sigma .
$$

Small values of $C L_{s+b}$ indicate poor compatibility with the signal+background hypothesis. Analogously, the confidence-level of the background only hypothesis is,

$$
C L_{b}=P_{b}\left(Q \leq Q_{o b s}\right),
$$

where values of $C L_{b}$ very close to one indicate poor compatibility with the background hypothesis. Agreement with the background hypothesis can also be characterized by the p-value given by, $p$-value $=1-C L_{b}$ in the modified frequentist formulation.

The modified frequentist method regards,

$$
C L_{S} \equiv \frac{C L_{s+b}}{C L_{b}}
$$

as the important confidence-level (actually it is a ratio of confidence-levels) and the signal hypothesis will be considered excluded at the confidence-level CL when,

$$
1-C L_{S} \leq C L
$$

The motivation for using $C L_{s}$ instead of $C L_{s+b}$ or $C L_{b}$ is that it avoids ambiguous situations where it would be difficult to clearly distinguish between the signal and signal+background hypotheses. It is also less sensitive to poor background modeling. 


\subsubsection{The Likelihood Ratio}

The likelihood ratio, given by Poisson statistics, satisfies the requirements of a teststatistic and is defined as,

$$
Q_{i}=\frac{L\left(s_{i}+b_{i}\right)}{L\left(b_{i}\right)}=\frac{e^{-\left(s_{i}+b_{i}\right)}\left(s_{i}+b_{i}\right)^{d_{i}}}{d_{i} !} / \frac{e^{-b_{i}} b_{i}^{d_{i}}}{d_{i} !}
$$

where $Q_{i}$ is the likelihood ratio of the i'th channel. The combined likelihood ratio for a data set, $Q_{d}$, is the product of all the likelihood ratios of the channels, $Q_{d}=\prod_{i=1}^{n} Q_{i}$. The estimate of the true cross-section is the one that minimizes Q. However, no significant excess is observed, therefore equation 6.3 will be used to set an upper limit. Due to the very low statistics that result after all the cuts are applied in this analysis, only the total number of events are considered in each counting experiment rather than considering each histogram bin as a separate counting experiment. In this situation the modified frequentist signal exclusion confidence becomes,

$$
C L=1-\frac{\sum_{n=0}^{n=d} \frac{e^{-(s+b)}(s+b)^{n}}{n !}}{\sum_{n=0}^{n=d e^{-b} b^{n}}}
$$

When uncertainties are included so that the number of signal events is $s \pm \sigma_{s}$ and the number of background events is $b \pm \sigma_{b}$, the probability to observe $n$ events is given by $[50]$,

$$
p_{s+b}^{n}=\frac{\int_{0}^{\infty} d s^{\prime} \int_{0}^{\infty} d b^{\prime} \frac{e^{-\left(\left(s^{\prime}-s\right)^{2} / 2 \sigma_{s}^{2}+\left(b^{\prime}-b\right)^{2} / 2 \sigma_{b}^{2}\right)} \frac{e^{-\left(s^{\prime}+b^{\prime}\right)}\left(s^{\prime}+b^{\prime}\right)^{n}}{n !}}{2 \pi \sigma_{s} \sigma_{b}}}{\int_{0}^{\infty} d s^{\prime} \int_{0}^{\infty} d b^{\prime} \frac{e^{-\left(\left(s^{\prime}-s\right)^{2} / 2 \sigma_{s}^{2}+\left(b^{\prime}-b\right)^{2} / 2 \sigma_{b}^{2}\right)}}{2 \pi \sigma_{s} \sigma_{b}}}
$$

and a similar expression is obtained for $p_{b}^{n}$. The probabilities can be inserted into the sums in equation 6.5 which is computed by the "top_statistics" software package [53]. Because equation 6.5 can become unwieldy, especially when systematics are included, 


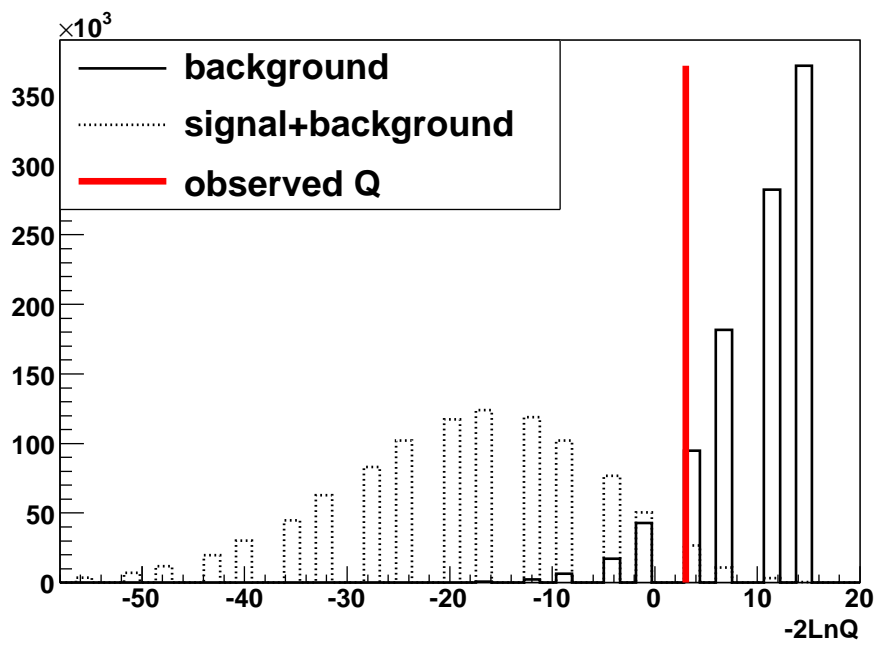

Figure 6.26: Distribution of -2LnQ for the background and signal+background hypotheses for 1 million MC pseudo-experiments. The observed value of $-2 \mathrm{LnQ}$ is indicated by the red line. In this example, $m_{H}=120 \mathrm{GeV}, m_{H V}=15 \mathrm{GeV}, \mathrm{dl}=50 \mathrm{~mm}$ is the signal. The area under the background distribution to the left of the observed $\mathrm{Q}$ is $1-C L_{b}$ and the area under the signal+background distribution to the right of the observed $\mathrm{Q}$ is $C L_{s+b}$.

the computation is done by generating $\mathrm{MC}$ ensembles corresponding to probability density functions for $s \pm \sigma_{s}$ and $b \pm \sigma_{b}$.

\subsubsection{Confidence-Limit Results}

The results of the $C L_{s}$ confidence-limits calculated by top_statistics are summarized in table 6.2 for each signal hypothesis. Figure 6.26 shows the -2LnQ distribution for an example signal and signal+background hypothesis. 


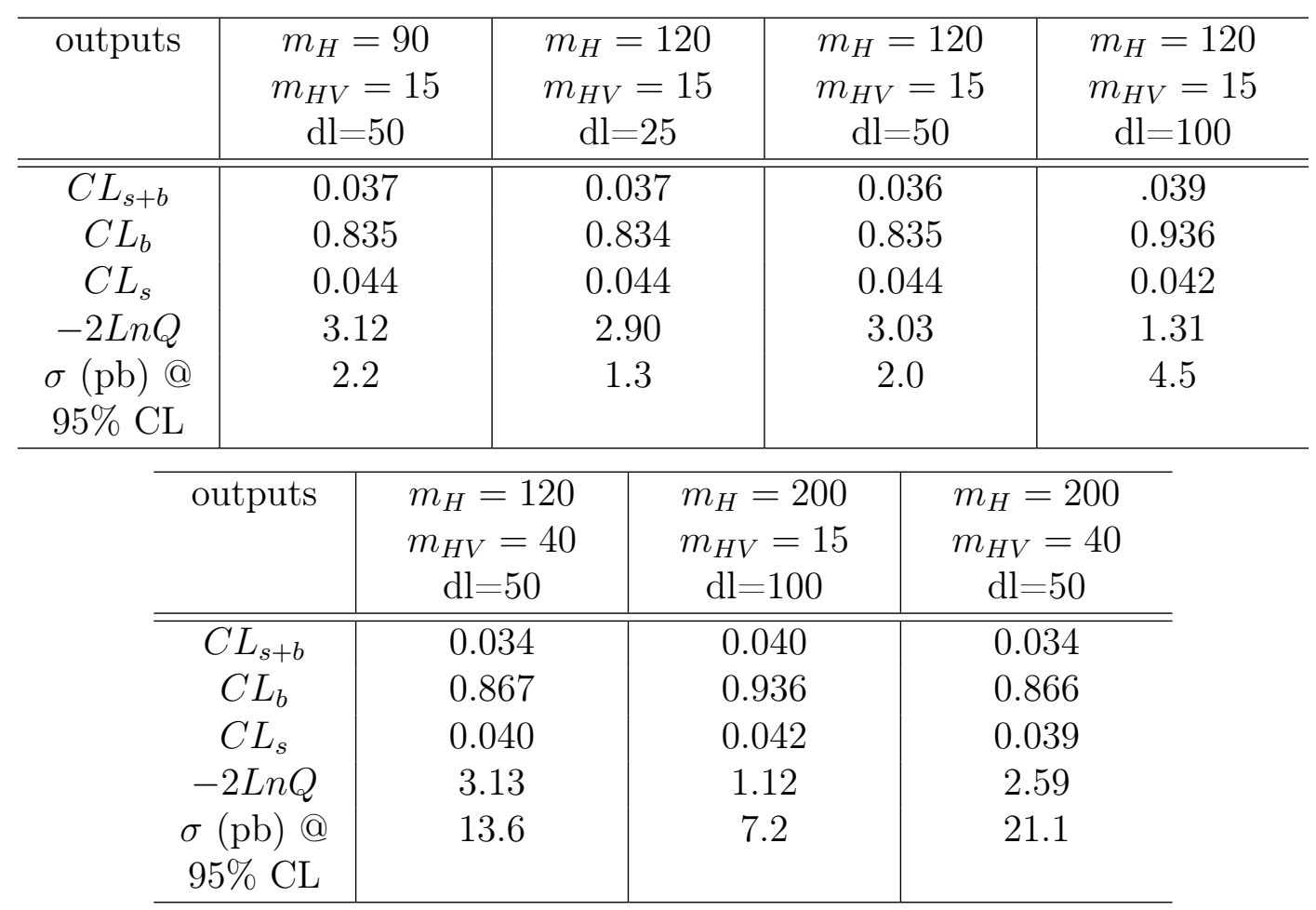

Table 6.2: The values of $C L_{s} @ 95 \%$ CL resulting from the top_statistics software package. The inputs come from section 6.4. 


\subsection{Results}

Tables 6.3, 6.4 and 6.5 summarize the cut-flow for the data, QCD MC and each signal hypothesis. The final plots of secondary-vertex mass and collinearity showing data versus MC for the signal hypotheses are shown in figures 6.27, 6.28 and 6.29 .

The final results are summarized in table 6.6 and 6.7 and displayed graphically in figure 6.30 and 6.31. Statistical uncertainties are the square root of the remaining number of unscaled MC events. Recall from section 5.2.2 that the QCD background is scaled by leading jet $p_{T}$ and number of primary-vertices to match the data, and normalized to data in section 6.2. Similarly, the signal events are scaled by crosssection and trigger efficiency. Limits are set using the modified-frequentist approach in the top_statistics software package [53] and cross-checked with the standard DØ limit setting procedure described in reference [52]. The observed limits at 95\% CL agree within $4 \%$ using either method. The p-value is the probability for the background to fluctuate up to the data. Although there is an excess number of observed events for each signal hypothesis, none of them is significant.

\subsubsection{Systematic Uncertainties}

The uncertainty on the luminosity measurement is the standard $6.5 \%$ which is included as a systematic uncertainty in the limit calculation. Uncertainty on the trigger efficiency comes from the muid_eff and jetid_eff packages and depends on the signal sample but on average the uncertainty is $14 \%$.

The uncertainty on the background model due to the difference in track reconstruction efficiency between MC and data is estimated by using two methods of nor- 


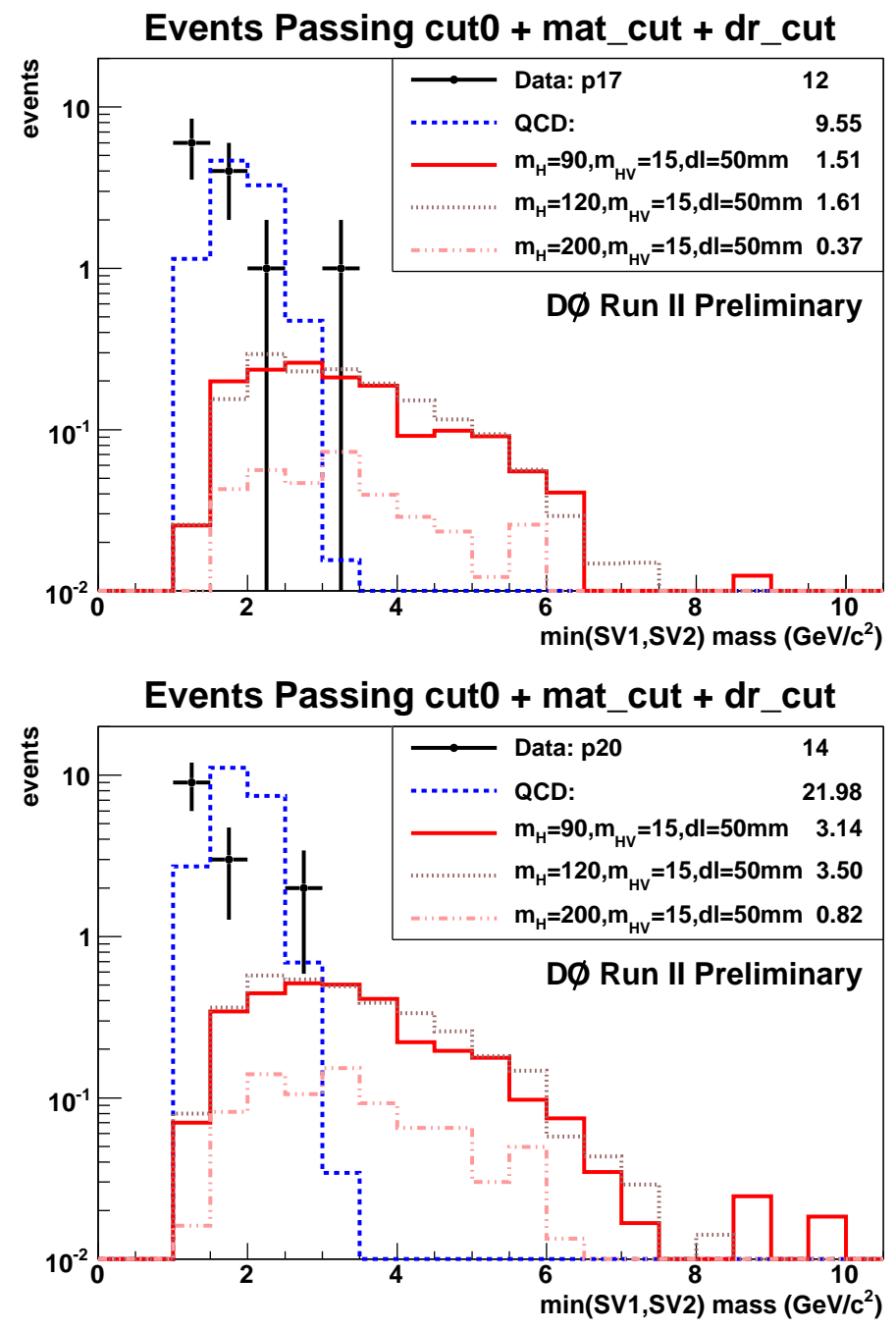

Figure 6.27: The minimum of the mass between the two secondary-vertices. This plot compares the data to the signal and background MC for three signal hypotheses for which mass is the optimal cut. 


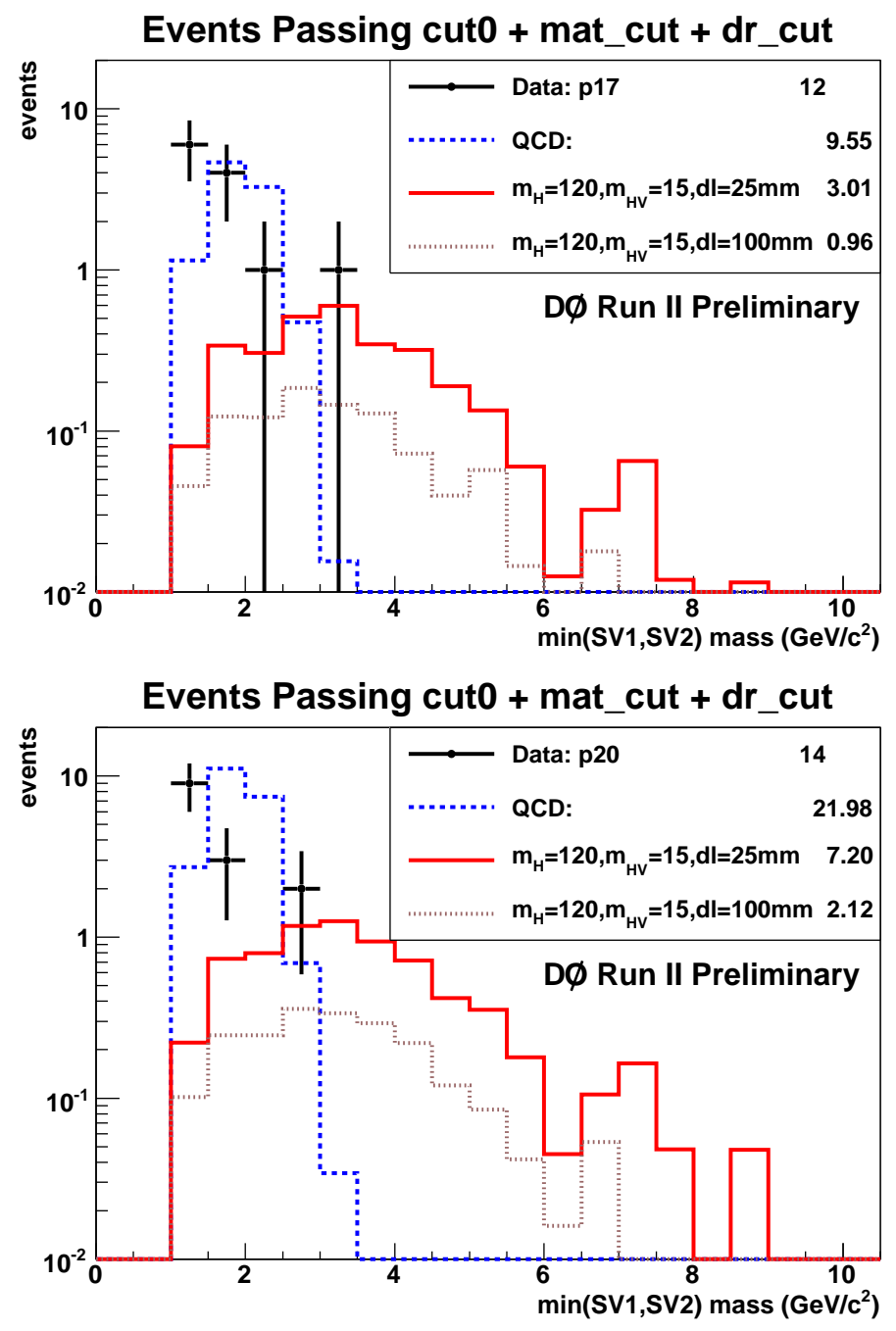

Figure 6.28: The minimum of the mass between the two secondary-vertices. This plot compares the data to the signal and background MC for two signal hypotheses for which mass is the optimal cut. 


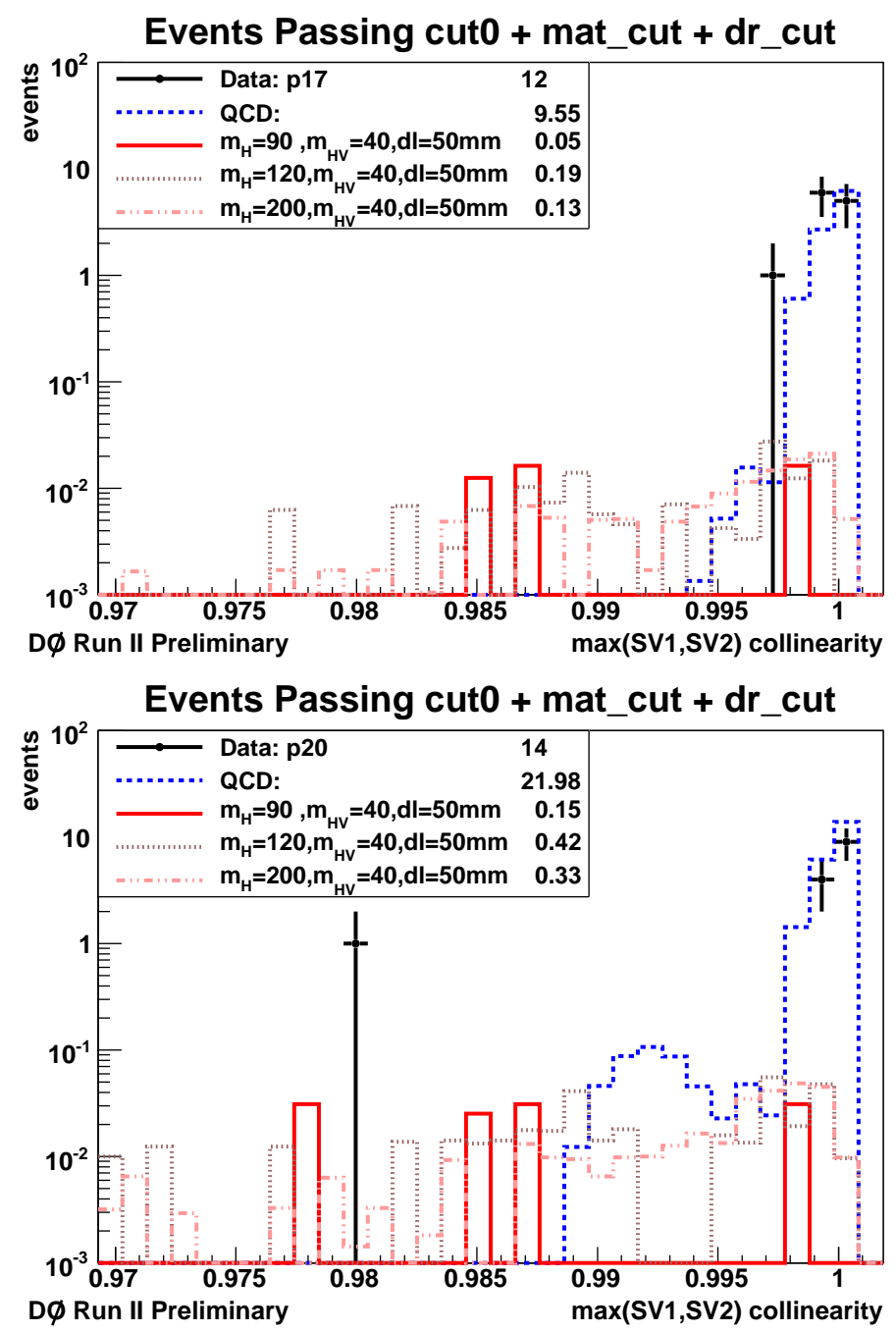

Figure 6.29: The maximum of the collinearity between the two secondary-vertices. This plot compares the data to the signal and background MC for two signal hypotheses for which collinearity is the optimal cut. 


\begin{tabular}{|c|c|c|c|c|c|}
\hline cut & QCD p17 & data p17 & $\begin{array}{c}m_{H}= \\
120 \\
m_{H V}= \\
15 \mathrm{dl}=50 \\
\text { p17 }\end{array}$ & $\begin{array}{l}m_{H}=120 \\
m_{H V}=15 \\
\mathrm{dl}=25 \mathrm{p} 17\end{array}$ & $\begin{array}{l}m_{H}=90 \\
m_{H V}=15 \\
\mathrm{dl}=50 \mathrm{p} 17\end{array}$ \\
\hline $\begin{array}{c}\text { pre-selection } \\
\text { num. } \mathrm{SV}>1.0 \\
\text { min mass }>2.5\end{array}$ & $\begin{array}{c}2.436 \times 10^{4} \\
9.55 \\
0.49 \\
\end{array}$ & $\begin{array}{c}2.436 \times 10^{4} \\
12 \\
1 \\
\end{array}$ & $\begin{array}{c}18.85 \\
1.61 \\
1.14 \\
\end{array}$ & $\begin{array}{c}24.35 \\
2.22 \\
1.69 \\
\end{array}$ & $\begin{array}{c}16.28 \\
1.51 \\
1.05 \\
\end{array}$ \\
\hline cut & QCD p20 & data p20 & $\begin{array}{l}m_{H}=120 \\
m_{H V}=15 \\
\mathrm{dl}=50 \mathrm{p} 20\end{array}$ & $\begin{array}{l}m_{H}=120 \\
m_{H V}=15 \\
\mathrm{dl}=25 \mathrm{p} 20\end{array}$ & $\begin{array}{c}m_{H}=90 \\
m_{H V}=15 \\
\mathrm{dl}=50 \mathrm{p} 20\end{array}$ \\
\hline $\begin{array}{c}\text { pre-selection } \\
\text { num. } \mathrm{SV}>1.0 \\
\text { min mass }>2.5\end{array}$ & $\begin{array}{c}3.560 \times 10^{4} \\
21.98 \\
0.73\end{array}$ & $\begin{array}{c}3.560 \times 10^{4} \\
14 \\
2\end{array}$ & $\begin{array}{c}39.02 \\
3.50 \\
2.49\end{array}$ & $\begin{array}{c}50.90 \\
5.30 \\
4.01\end{array}$ & $\begin{array}{l}29.32 \\
3.14 \\
2.29\end{array}$ \\
\hline
\end{tabular}

Table 6.3: Summary of the cut-flow which is optimized for the signal hypotheses $\left(m_{H}=120, m_{H V}=15, d l=50 \mathrm{~mm}\right),\left(m_{H}=120, m_{H V}=15, d l=50 \mathrm{~mm}\right)$ and $\left(m_{H}=90, m_{H V}=15, d l=50 \mathrm{~mm}\right)$. QCD is normalized to data after the preselection.

\begin{tabular}{|c|c|c|c|c|}
\hline cut & QCD p17 & data p17 & $\begin{array}{c}m_{H}=120 \\
m_{H V}=15 \\
\mathrm{dl}=100 \\
\text { p17 }\end{array}$ & $\begin{array}{l}m_{H}=200 \\
m_{H V}=15 \\
\mathrm{dl}=50 \mathrm{p} 17\end{array}$ \\
\hline $\begin{array}{c}\text { pre-selection } \\
\text { num. }>1.0 \\
\text { min mass }>3.0\end{array}$ & $\begin{array}{c}2.436 \times 10^{4} \\
9.55 \\
0.02 \\
\end{array}$ & $\begin{array}{c}2.436 \times 10^{4} \\
12 \\
1 \\
\end{array}$ & $\begin{array}{c}11.84 \\
0.65 \\
0.33 \\
\end{array}$ & $\begin{array}{l}5.78 \\
0.37 \\
0.21 \\
\end{array}$ \\
\hline cut & QCD p20 & data p20 & $\begin{array}{c}m_{H}=120 \\
m_{H V}=15 \\
\mathrm{dl}=100 \\
\mathrm{p} 20\end{array}$ & $\begin{array}{l}m_{H}=200 \\
m_{H V}=15 \\
\mathrm{dl}=50 \mathrm{p} 20\end{array}$ \\
\hline $\begin{array}{c}\text { pre-selection } \\
\text { num. }>1.0 \\
\text { min mass }>3.0\end{array}$ & $\begin{array}{c}3.560 \times 10^{4} \\
21.98 \\
0.04\end{array}$ & $\begin{array}{c}3.560 \times 10^{4} \\
14 \\
0\end{array}$ & $\begin{array}{c}24.28 \\
1.44 \\
0.79\end{array}$ & $\begin{array}{c}11.92 \\
0.82 \\
0.48\end{array}$ \\
\hline
\end{tabular}

Table 6.4: Summary of the cut-flow which is optimized for the signal hypotheses $\left(m_{H}=120, m_{H V}=15, d l=100 \mathrm{~mm}\right)$ and $\left(m_{H}=200, m_{H V}=15, d l=50 \mathrm{~mm}\right)$. QCD is normalized to data after the pre-selection. 

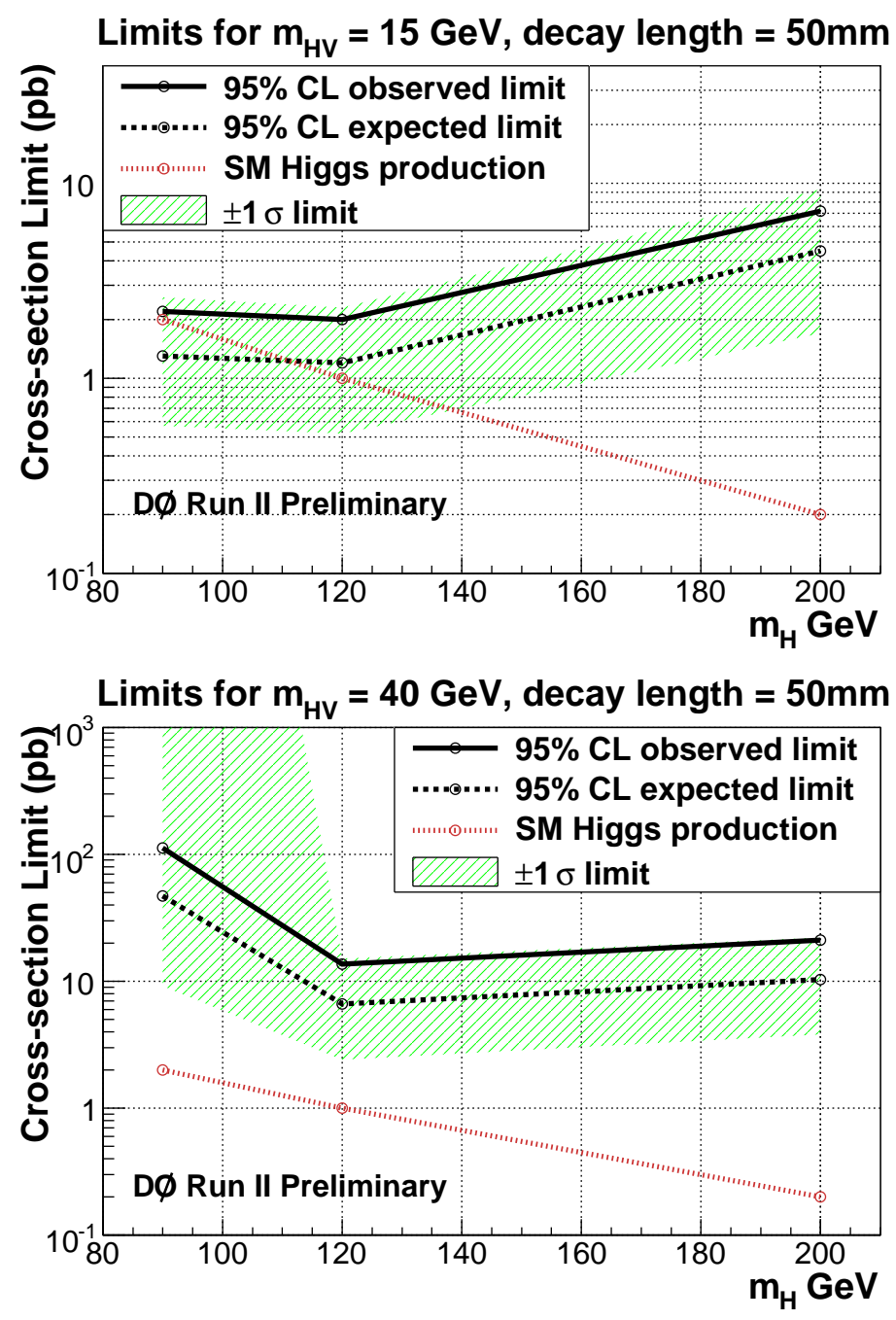

Figure 6.30: Observed and expected cross-sections as a function of Higgs boson mass. 


\begin{tabular}{|c|c|c|c|c|c|}
\hline cut & QCD p17 & data p17 & $\begin{array}{l}m_{H}=90 \\
m_{H V}=40 \\
\mathrm{dl}=50 \mathrm{p} 17\end{array}$ & $\begin{array}{l}m_{H}=120 \\
m_{H V}=40 \\
\mathrm{dl}=50 \mathrm{p} 17\end{array}$ & $\begin{array}{l}m_{H}=200 \\
m_{H V}=40 \\
\mathrm{dl}=50 \mathrm{p} 17\end{array}$ \\
\hline $\begin{array}{c}\text { pre-selection } \\
\text { num. }>1.0 \\
\text { max coll. }<0.9977\end{array}$ & $\begin{array}{c}2.436 \times 10^{4} \\
9.55 \\
0.034\end{array}$ & $\begin{array}{c}2.436 \times 10^{4} \\
12 \\
1\end{array}$ & $\begin{array}{l}1.76 \\
0.05 \\
0.03\end{array}$ & $\begin{array}{l}4.67 \\
0.19 \\
0.16\end{array}$ & $\begin{array}{l}3.15 \\
0.13 \\
0.09\end{array}$ \\
\hline cut & QCD p20 & data p20 & $\begin{array}{l}m_{H}=90 \\
m_{H V}=40 \\
\mathrm{dl}=50 \mathrm{p} 20\end{array}$ & $\begin{array}{l}m_{H}=120 \\
m_{H V}=40 \\
\mathrm{dl}=50 \mathrm{p} 20\end{array}$ & $\begin{array}{l}m_{H}=200 \\
m_{H V}=40 \\
\mathrm{dl}=50 \mathrm{p} 20\end{array}$ \\
\hline $\begin{array}{c}\text { pre-selection } \\
\text { num. }>1.0 \\
\text { max coll. }<0.9977\end{array}$ & $\begin{array}{c}3.560 \times 10^{4} \\
21.98 \\
0.48\end{array}$ & $\begin{array}{c}3.560 \times 10^{4} \\
14 \\
1\end{array}$ & $\begin{array}{l}3.72 \\
0.15 \\
0.12\end{array}$ & $\begin{array}{l}9.42 \\
0.42 \\
0.34\end{array}$ & $\begin{array}{l}6.97 \\
0.33 \\
0.23\end{array}$ \\
\hline
\end{tabular}

Table 6.5: Summary of the cut-flow which is optimized for the signal hypotheses $\left(m_{H}=120, m_{H V}=40, d l=50 \mathrm{~mm}\right)$ and $\left(m_{H}=200, m_{H V}=40, d l=50 \mathrm{~mm}\right)$. QCD is normalized to data after the pre-selection.

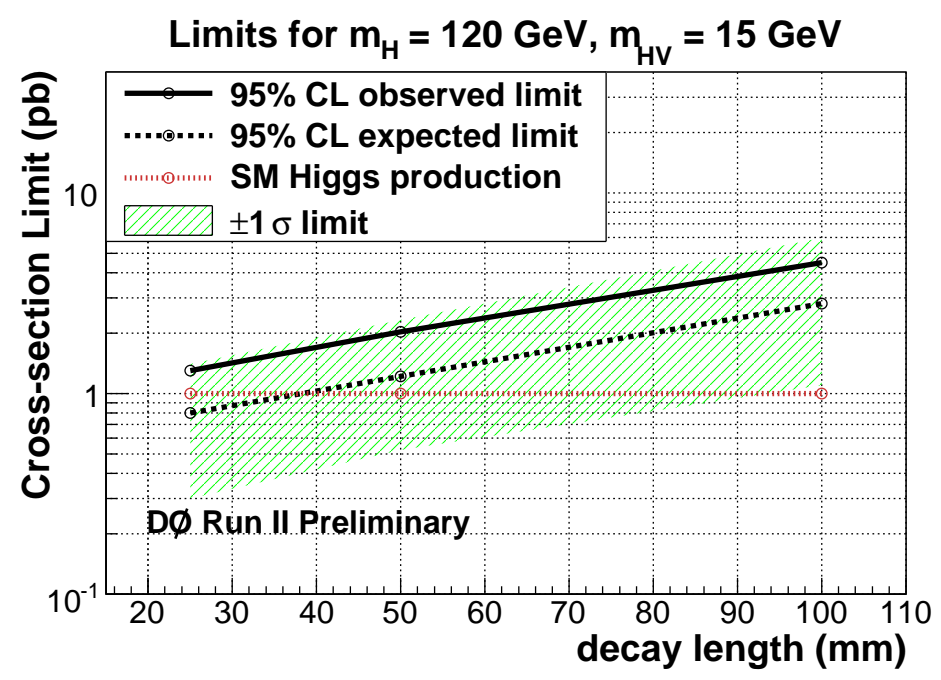

Figure 6.31: Observed and expected cross-sections as a function of HV particle decay length. 


\begin{tabular}{|c|c|c|}
\hline & $\begin{array}{c}m_{H}=200 \\
m_{H V}=15 \mathrm{dl}=50\end{array}$ & $\begin{array}{c}m_{H}=200 \\
m_{H V}=40 \mathrm{dl}=50\end{array}$ \\
\hline background & $\begin{array}{c}0.061 \pm \\
100 \%(\text { stat }) \\
\pm 35 \%(\text { syst })\end{array}$ & $\begin{array}{c}0.515 \pm 40 \% \text { (stat) } \\
\pm 91 \% \text { (syst) }\end{array}$ \\
\hline signal & $\begin{array}{c}0.691 \pm 8 \%(\text { stat }) \\
\pm 13 \%(\text { syst })\end{array}$ & $\begin{array}{c}0.310 \pm 12 \% \text { (stat) } \\
\pm 15 \% \text { (syst) }\end{array}$ \\
\hline $\begin{array}{c}\text { data }(\mathrm{p} 17+\mathrm{p} 20) \\
\text { obs. limit @95\%CL } \\
\text { p-value/significance } \\
\text { exp. limit @95\%CL }\end{array}$ & $\begin{array}{c}1 \\
7.2 \mathrm{pb} \\
6 \% / 1.5 \sigma \\
4.5 \mathrm{pb}\end{array}$ & $\begin{array}{c}2 \\
21.1 \mathrm{pb} \\
13 \% / 1.1 \sigma \\
10.3 \mathrm{pb}\end{array}$ \\
\hline SM Higgs production & \multicolumn{2}{|l|}{$0.2 \mathrm{pb}$} \\
\hline & $\begin{array}{c}m_{H}=120 \\
m_{H V}=15 \mathrm{dl}=50\end{array}$ & $\begin{array}{c}m_{H}=120 \\
m_{H V}=40 \mathrm{dl}=50\end{array}$ \\
\hline background & $\begin{array}{c}1.22 \pm 53 \%(\text { stat }) \\
\pm 35 \%(\text { syst })\end{array}$ & $\begin{array}{c}0.515 \pm 40 \% \text { (stat) } \\
\pm 91 \% \text { (syst) }\end{array}$ \\
\hline signal & $\begin{array}{c}3.63 \pm 7 \% \text { (stat) } \\
\pm 15 \% \text { (syst) }\end{array}$ & $\begin{array}{c}0.506 \pm 17 \% \text { (stat) } \\
\pm 17 \% \text { (syst) }\end{array}$ \\
\hline $\begin{array}{c}\text { data }(\mathrm{p} 17+\mathrm{p} 20) \\
\text { obs. limit @95\%CL } \\
\text { p-value/significance } \\
\text { exp. limit @95\%CL }\end{array}$ & $\begin{array}{c}3 \\
2.0 \mathrm{pb} \\
16 \% / 1.0 \sigma \\
1.2 \mathrm{pb} \\
\end{array}$ & $\begin{array}{c}2 \\
13.6 \mathrm{pb} \\
13 \% / 1.1 \sigma \\
6.6 \mathrm{pb} \\
\end{array}$ \\
\hline \multirow[t]{2}{*}{ SM Higgs production } & \multicolumn{2}{|l|}{1.0} \\
\hline & $\begin{array}{c}m_{H}=120 \\
m_{H V}=15 \mathrm{dl}=25\end{array}$ & $\begin{array}{c}m_{H}=120 \\
m_{H V}=15 \\
\mathrm{dl}=100\end{array}$ \\
\hline background & $\begin{array}{l}1.22 \pm 53 \%(\text { stat }) \\
\quad \pm 35 \%(\text { syst })\end{array}$ & $\begin{array}{c}0.061 \pm \\
100 \%(\text { stat }) \\
\pm 35 \%(\text { syst })\end{array}$ \\
\hline signal & $\begin{array}{c}5.70 \pm 6 \% \text { (stat) } \\
\pm 13 \% \text { (syst) }\end{array}$ & $\begin{array}{c}1.12 \pm 12 \%(\text { stat }) \\
\pm 13 \%(\text { syst })\end{array}$ \\
\hline $\begin{array}{l}\text { data }(\mathrm{p} 17+\mathrm{p} 20) \\
\text { obs. limit @ } 95 \% \mathrm{CL} \\
\text { p-value/significance } \\
\text { exp. limit @ } 95 \% \mathrm{CL}\end{array}$ & $\begin{array}{c}3 \\
1.3 \mathrm{pb} \\
16 \% / 1.0 \sigma \\
0.8 \mathrm{pb}\end{array}$ & $\begin{array}{c}1 \\
4.5 \mathrm{pb} \\
6 \% / 1.5 \sigma \\
2.8 \mathrm{pb}\end{array}$ \\
\hline SM Higgs production & $1.0 \mathrm{pb}$ & \\
\hline
\end{tabular}

Table 6.6: Summary of results for six signals hypotheses. Data from p17 and p20 are combined. 


\begin{tabular}{c|c|c}
\hline & $m_{H}=90$ & $m_{H}=90$ \\
& $m_{H V}=15 \mathrm{dl}=50$ & $m_{H V}=40 \mathrm{dl}=50$ \\
\hline \hline background & $1.22 \pm$ & $0.515 \pm 40 \%($ stat $)$ \\
& $53 \%($ stat $) \pm 35 \%$ (syst) $\pm 91 \%($ syst $)$ \\
\hline signal & $3.34 \pm$ & $0.15 \pm 47 \%($ stat $)$ \\
& $8 \%($ stat $) \pm 15 \%$ (syst $)$ & $\pm 17 \%($ syst $)$ \\
\hline data (p17+p20) & 3 & 2 \\
obs. limit @95\%CL & $2.2 \mathrm{pb}$ & $112.3 \mathrm{pb}$ \\
p-value/significance & $16 \% / 1.0 \sigma$ & $13 \% / 1.1 \sigma$ \\
exp. limit @95\%CL & $1.3 \mathrm{pb}$ & $46.9 \mathrm{pb}$ \\
\hline SM Higgs production & $2.0 \mathrm{pb}$ & $2.0 \mathrm{pb}$ \\
\hline
\end{tabular}

Table 6.7: Summary of results for the last two signal hypotheses. Data from p17 and p20 are combined.

malization. In the first method we simply normalize the number of QCD events to the number of data events after cut0+mat_cut (these cuts are defined in table 6.1) In the second method we reweight the QCD track multiplicity to match the data and then multiply this event weight by the event weight which is used to match leading jet $p_{T}$ and number of primary-vertices (refer to section 5.2.2). Figure 6.32 shows the agreement after this operation. After requiring two SVs per event, the two methods are compared and the resulting number of QCD events differ by 34\%, which is added as a systematic uncertainty on the background model.

We estimate the effect of smearing the MC samples by performing the whole analysis without smearing. For the cuts applied to the collinearity, smearing results in a difference of $83 \%$ on the background QCD MC and no difference for the signal MC. Smearing also has no effect on the outcome of the cuts which are applied to the mass.

The secondary-vertex density is poorly modeled in the $x y$-plane. To estimate the effect of cutting on this variable, we look at the difference in remaining events between 


\begin{tabular}{c|c}
\hline quantity & error \\
\hline \hline luminosity & $6.5 \%$ \\
trigger efficiency (average) & $14 \%$ \\
background multiplicity & $34 \%$ \\
smearing collinearity (QCD only) & $84 \%$ \\
$x y$-density modeling & p20: $8 \% \mathrm{p} 17: 5 \%$ \\
\hline
\end{tabular}

Table 6.8: Summary of the systematic uncertainties.

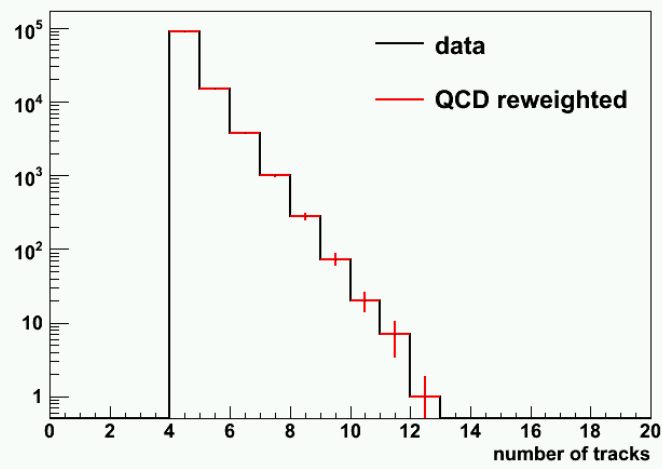

Figure 6.32: Comparison of QCD to data after reweighting the QCD track multiplicity to match the data. This is the combined p17 and p20 data set.

QCD and data after making the density cut, refer to figure 6.33. We estimate the difference to be $8 \%$ for p20 and $5 \%$ for p17.

\subsubsection{Material Interaction Cross Check}

After performing the analysis we check the locations of the data events to see if they happen to fall near any detector material (refer to figure 6.34 and 6.35). The material regions are defined as areas of high SV density. Cuts are made on the density of SVs in the $x y$ and $z r$ planes, as discussed in section 6.2. The result of the cross check shows that the data events that passed all of the cuts are not close to the SV density cut, which is to say, they are not typically distributed close to any high density material 

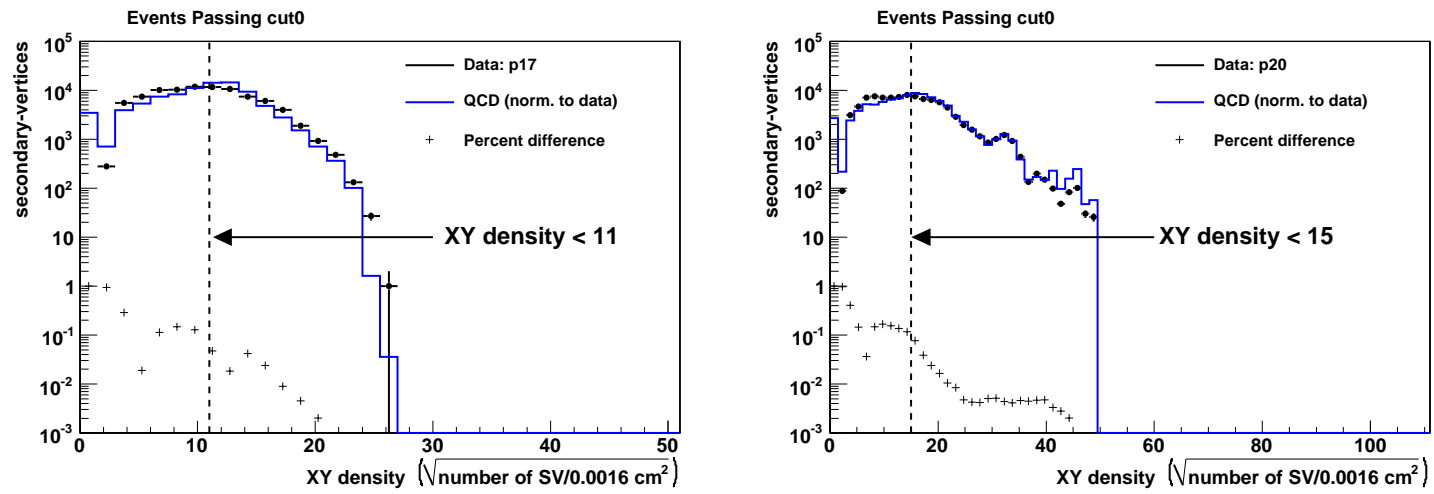

Figure 6.33: Secondary-vertex density in the $x y$-plane for data and QCD where QCD is normalized to data. The black crosses are the percent difference of remaining events between QCD and data if a cut is made at the given value. The dashed lines show the locations of the actual cuts that are made.

regions.

\subsection{Summary}

A search for neutral, long-lived particles decaying to $b$-jets is presented. Long-lived, electrically neutral hadrons of this nature have recently been proposed in hiddenvalley models and may provide a novel path to Higgs discovery at the Tevatron. We analyze $3.65 \mathrm{fb}^{-1}$ of data for HV particles that could be produced from a Higgs decay. Eight signal hypotheses are analyzed: $\left(m_{H}=90 \mathrm{GeV}, m_{H V}=15 \mathrm{GeV}\right.$, decay length $=50 \mathrm{~mm}),\left(m_{H}=120 \mathrm{GeV}, m_{H V}=15 \mathrm{GeV}\right.$, decay length $\left.=25 \mathrm{~mm}\right),\left(m_{H}=120\right.$ $\mathrm{GeV}, m_{H V}=15 \mathrm{GeV}$, decay length $\left.=50 \mathrm{~mm}\right),\left(m_{H}=120 \mathrm{GeV}, m_{H V}=15 \mathrm{GeV}\right.$, decay length $=100 \mathrm{~mm}),\left(m_{H}=120 \mathrm{GeV}, m_{H V}=40 \mathrm{GeV}\right.$, decay length $=50$ $\mathrm{mm}),\left(m_{H}=200 \mathrm{GeV}, m_{H V}=15 \mathrm{GeV}\right.$, decay length $\left.=50 \mathrm{~mm}\right)$ and $\left(m_{H}=200\right.$ $\mathrm{GeV}, m_{H V}=40 \mathrm{GeV}$, decay length $=50 \mathrm{~mm}$ ) for which no significant excess is observed and the respective cross-sections of $2.2,1.3,2.0,4.5,13.6,7.2$, and $21.1 \mathrm{pb}$ 

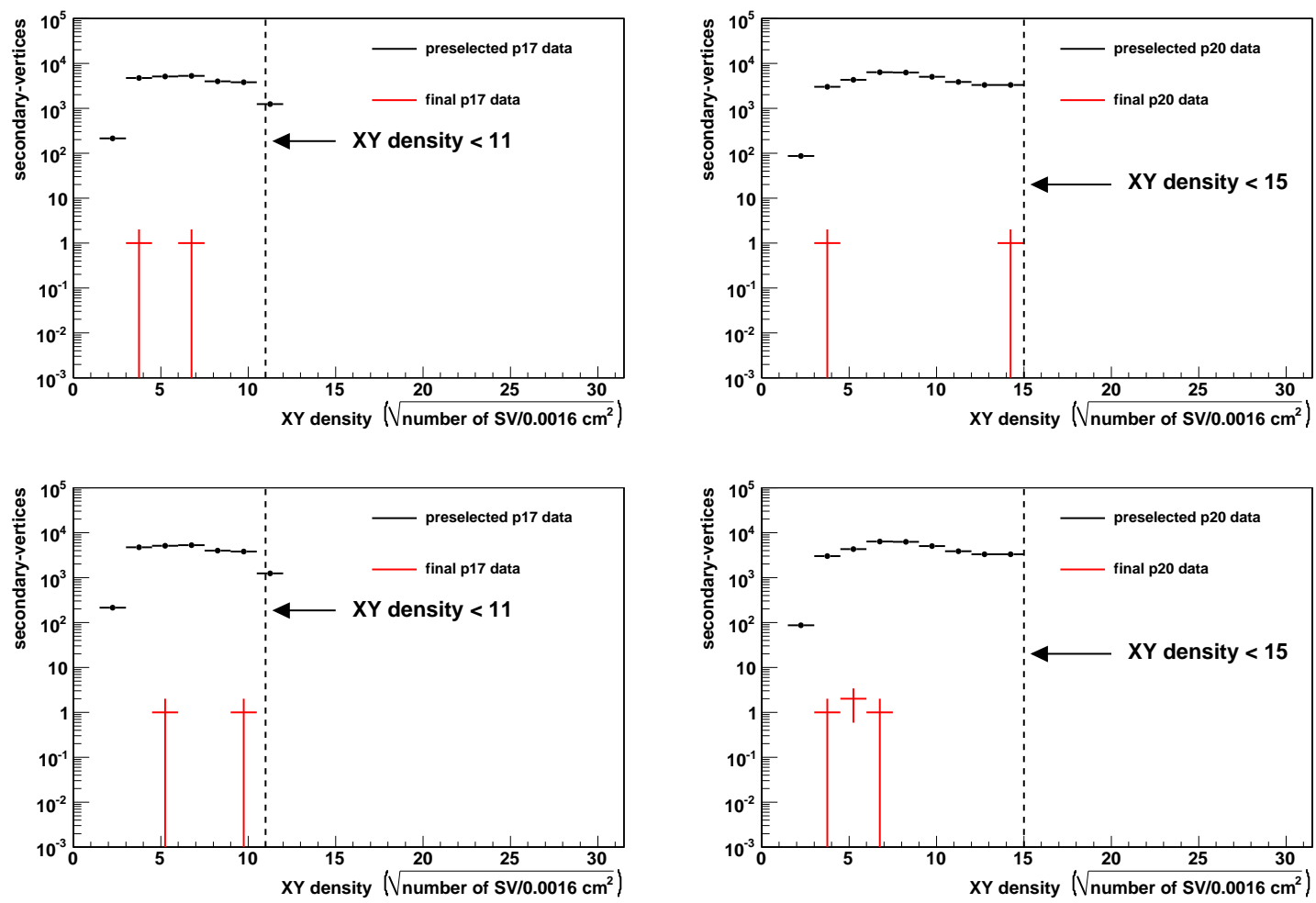

Figure 6.34: The SV density in the $x y$ plane for data secondary-vertices (two SVs per event). The red lines are data events resulting from the cuts used to search for the signal points while the black lines are the pre-selected data. 

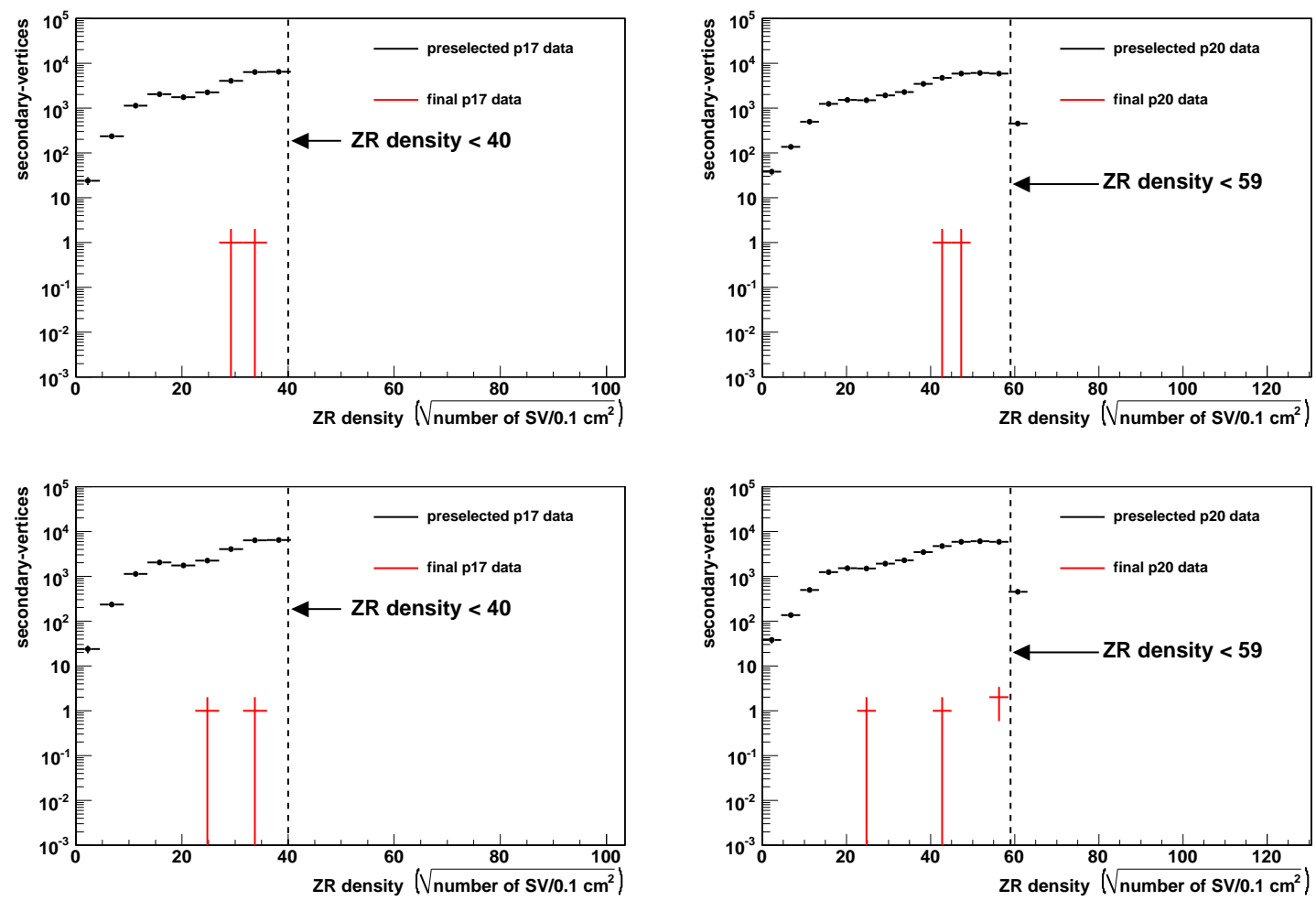

Figure 6.35: The SV density in the $z r$ plane for data secondary-vertices (two SVs per event). The red lines are data events resulting from the cuts used to search for the signal points while the black lines are the pre-selected data. 
are excluded at 95\% CL. 


\section{Bibliography}

[1] David J. Griffiths. Introduction to Elementary Particles. John Wiley \& Sons, Inc., 1987. (Cited on page 6.)

[2] Michael E. Peskin, Daniel V. Schroeder. An Introduction to Quantum Field Theory. Westview Press, 1995. (Cited on page 6.)

[3] Ta-Pei Cheng, Ling-Fong Li. Gauge Theory of Elementary Particle Physics. Clarendon Press, 2002. (Cited on page 6.)

[4] C. Amsler, et al. Review of Paricle Physics. Phys. Lett. B, 667:1-1340, 2008. http://pdg.lbl.gov. (Cited on pages 12 and 15.)

[5] ALEPH, DELPHI, L3, and OPAL Collaborations. The LEP Working Group for Higgs Boson Searches. Phys. Lett., B565:61-75, 2003. arxiv:hep-ex/0306033. (Cited on page 13.)

[6] CDF and D $\varnothing$ Collaborations. Combined CDF and D $\varnothing$ Upper Limits on Standard Model Higgs-Boson Production with up to $4.2 \mathrm{fb}^{-1}$ of data. DØ Note 5754, 2008. (Cited on page 13.)

[7] G. Abbiendi, et al. Decay-Mode Independent Searches for New Scalar Bosons with the OPAL Detector at LEP. Eur. Phys. J., C27:311, 2003. arxiv:hepex/0206022. (Cited on page 13.)

[8] G. Abbiendi, et al. (the ALEPH Collaboration, the DELPHI Collaboration, the L3 Collaboration and the OPAL Collaboration, The LEP Working Group for Higgs Boson Searches). Search for the Standard Model Higgs Boson at LEP. Phys. Lett. B, 565:61-75, 2003. arxiv:hep-ex/0306033v1. (Cited on page 13.)

[9] LEP Electroweak Working Group. LEP EW WG Plots for Summer 2008. http://lepewwg.web.cern.ch/LEPEWWG/plots/summer2008/. (Cited on page 14.) 
[10] The D $\varnothing$ Collaboration. Search for $W H$ Associated Production Using a Combined Neural Network and Matrix Element Approach with $2.7 \mathrm{fb}^{-1}$ of Run II Data. February 2009. DØ Note 5828-CONF. (Cited on page 16.)

[11] Spencer Chang, Patrick J. Fox, Neal Weiner. Naturalness and Higgs Decays in the MSSM with a Singlet. arxiv:hep-ph/0511250v2. (Cited on page 16.)

[12] The DØ Collaboration. Long-Lived Particles in the Dimuon Channel with 383 $p b^{-1}$ of Run II Data Search for Neutral. 2006. DØ Note 5023-CONF. (Cited on pages 16 and 21.)

[13] F. Abe, et al. [The CDF Collaboration]. Search for Long-Lived Parents of $Z^{0}$ Bosons in $\mathrm{p} \bar{p}$ Collisions at $\sqrt{s}=1.8 \mathrm{TeV}$. Phys. Rev. D, 58, 1998. arxiv:hepex/9805017. (Cited on page 16.)

[14] T. Aaltonen, et al. [The CDF Collaboration]. Study of Multi-Muon Events Produced in $\mathrm{p} \bar{p}$ Collisions at $\sqrt{s}=1.8 \mathrm{TeV}$. arxiv:hep-ex/0810.5357v2. (Cited on page 16.)

[15] The DØ Collaboration. Search for Excess Dimuon Production in the Radial Region $1.6<\mathrm{r}<7.6 \mathrm{~cm}$ at the DØ Experiment. DØ Note 5905-CONF. (Cited on page 16.)

[16] Matthew J. Strassler. Flesh and Blood or Merely Ghosts? Some Comments on the Multi-Muon Study at CDF. arxiv:0811.1560v2. (Cited on page 16.)

[17] Matthew J. Strassler, Kathryn M. Zurek. Echoes of a Hidden Valley at Hadron Colliders. Phys. Lett., B651:374-379, 2007. arXiv:hep-ph/0604261. (Cited on pages 17, 18 and 19.)

[18] Matthew J. Strassler, Kathryn M. Zurek. Discovering the Higgs Through HighlyDisplaced Vertices. Phys. Lett., B661:263-267, 2008. arXiv:hep-ph/0605193. (Cited on pages 18 and 19.)

[19] Thomas G. Rizzo. "Z' Phenomenology and the LHC". October 2006. arXiv:hepph/0408098v1. (Cited on page 19.)

[20] Emmanuel Busato, Bernard Andrieu. Jet Algorithms in the DØ Run II Software: Description and User's Guide. DØ Note 4457. (Cited on pages 28 and 59.)

[21] The DØ Collaboration. Run II Handbook. http://wwwbd.fnal.gov/lug/runII_handbook/RunII_index.html. (Cited on page 29.) 
[22] Joey Thompson. Introduction to Colliding Beams at Fermilab. FERMILABTM-1909, 1994. (Cited on pages 29 and 30.)

[23] S. Mishra. High Luminosity Operation of the Fermilab Aceelerator Complex. Proceedings of the 2003 Particle Accelerator Conference. (Cited on pages 29 and 31.)

[24] V.M. Abazov, et al. The Upgraded DØ Detector. NIM, A565:463-537, 2006. (Cited on pages 33, 39, 40, 41 and 44.)

[25] Michael Begel. Luminosity, 2003. http://www-d0.fnal.gov/runcoor/DAQ/. (Cited on page 36.)

[26] Michele Weber. The DØ Silicon Microstrip Tracker, 2004. Vertex 2004. (Cited on page 37.)

[27] D $\varnothing$ Silicon Tracker Drawings and Photos. http://d0server1.fnal.gov/projects/silicon/www/drawings_eng.html. (Cited on page 37.)

[28] Andy Haas. A Search for Neutral Higgs Bosons at High tan $\beta$ in Multi-jet Events from p $\bar{p}$ Collisions at $\sqrt{s}=1960 \mathrm{GeV}$. PhD thesis, University of Washington, 2004. (Cited on pages 43 and 50.)

[29] M. Abolins, et al. The Run IIb Trigger Upgrade for the DØ Experiment. IEEE Trans. on Nucl. Sci., 51:340-344, 2004. http://www.nevis.columbia.edu/ evans/11cal/docs/docs.html. (Cited on page 47.)

[30] R. Demina, J. Hernandez, A. Khanov, F. Rizatdinova, A. Nomerotski. Verification of the Lorentz Angle Value Using SMT Data. 2002. DØ Note 3945. (Cited on page 50.)

[31] Michael Kirby. SMT DAQ Shifter Tutorial. 2007. http://wwwd0.fnal.gov/runcoor/DAQ/Tutorials/2006/2006-02-07_Kirby_SMT.pdf. (Cited on page 50 .)

[32] P.V.C. Hough. Machine Analysis of Bubble Chamber Pictures. Int. Conf. on High Energy Accelerators and Instrumentation, 1959. CERN. (Cited on page 51.)

[33] Alexander Khanov. HTF: Histogramming Method for Finding Tracks. The Algorithm Description. 2000. DØ Note 3778. (Cited on pages 51 and 52.) 
[34] R. Frühwirth. Application of Kalman Filtering to Track and Vertex Fitting. Nucl. Inst. and Meth. in Phys. Res., A262:444-450, 1987. (Cited on page 51.)

[35] H. Greenlee. The DØ Kalman Track Fit. 2004. DØ Note 4303. (Cited on page 51.)

[36] G. Borissov. Ordering a Chaos or... Technical Details of AA Tracking. 2003. http://www-d0.fnal.gov/atwork/adm/d0_private/2003-0228/adm_talk.ps. (Cited on page 51.)

[37] A. Schwartzman, C. Tully. Primary Vertex Reconstruction by Means of Adaptive Vertex Fitting. 2005. DØ Note 4918. (Cited on page 54.)

[38] A. Schwartzman, M. Narain. Secondary Vertex Reconstruction using the Kalman Filter. 2001. DØ Note 3908. (Cited on page 56.)

[39] Aurelio Juste, Christophe Royon. DØ Preliminary Jet Energy Scale. http://www-d0.fnal.gov/phys_id/jes/public/plots_v7.1/. (Cited on pages 58 and 61.)

[40] Gerald C. Blazey, et al. Run II Jet Physics. 2000. arxiv:hep-ex/0005012v2. (Cited on pages 60 and 61.)

[41] Amnon Harel. Jet ID Optimization. DØ Note 4919. (Cited on page 60.)

[42] Philippe Calfayan, et al. Muon Identification Certification for p17 Data. DØ Note 5157. (Cited on pages 63, 64 and 74.)

[43] DØ Data Quality Coordination. Finding out what is good / bad Data. http://www-d0.fnal.gov/computing/data_quality/d0_private/forusers.html. (Cited on pages 65 and 66. )

[44] T. Sjöstrand, et al. Phythia 6.3 Physics and Manual. arxiv:hep-ph/0308153. (Cited on page 67.)

[45] T. Hahn, S. Heinemeyer, F. Maltoni, G. Weiglein, S. Willenbrock. SM, MSSM Higgs Boson Production Cross Sections at the Tevatron, and the LHC. 2006. arXiv:hep-ph/0607308v2. (Cited on page 73.)

[46] Amnon Harel, et al. p20 JetID Efficiencies and Scale Factors. DØ note 5634. (Cited on page 74.)

[47] Philippe Calfayan, Boris Tuchming, et al. muid_eff package. http://wwwd0.fnal.gov/d0dist/dist/releases/development/muid_eff/doc/html/. (Cited on page 75.$)$ 
[48] J. Friedman. Procedings of the 1974 CERN School of Computing, Norway, 1974. (Cited on page 99.)

[49] A L Read. Presentation of Search Reults: the $C L_{S}$ Technique. J. Phys. G: Nucl. Part. Phys., 28:2693-2704, 2002. (Cited on page 109.)

[50] Thomas Junk. Confidence Level Computation for Combining Searches with Small Statistics. CARLETON/OPAL PHYS 99-01, 1999. arxiv:hepex/9902006v1. (Cited on pages 109 and 111.)

[51] A. L. Read. Modified Frequentist Analysis of Search Results (The $C L_{S}$ Method). 1st Workshop on Confidence Limits, 2000. CERN-OPEN-2000-205. (Cited on page 109.)

[52] I Bertram, G. Landsberg, J. Linnemann, R. Partridge, M. Paterno, H.B. Prosper. A Recipe for the Construction of Confidence Limits. D $\varnothing$ Note 3476. (Cited on pages 109 and 114.)

[53] Supriya Jain, Harrison B. Prosper, Reinhard Schwienhorst. Statistical Methods Implemented in the Package "top_statistics". December 2008. D0 Note 5817. (Cited on pages 111 and 114.)

[54] Yuri Gershtein. DØ Results from Run 2: Wine \& Cheese, July 26 2002. (Not cited.)

[55] Matthew J. Strassler. On the Phenomenology of Hidden Valleys with Heavy Flavor. 2008. arXiv:hep-ph/0806.2385v1. (Not cited.)

[56] Matthew J. Strassler. Possible Effects of a Hidden Valley on Supersymmetric Phenomenology. 2006. arXiv:hep-ph/0607160v1. (Not cited.)

[57] Supriya Jain, Harrison B. Prosper, Brigitte Vachon, Reinhard Schwienhorst, and Daekwang Kau. Limits using a Bayesian approach in the package "top_statistics". DØ Note 5123, 2006. (Not cited.)

[58] Tao Han, Kathryn M. Zurek, Matthew J. Strassler. Phenomenology of Hidden Valleys at Hadron Colliders. 2007. arXiv:hep-ph/0712.2041v2. (Not cited.) 


\section{Appendix A}

\section{Trigger Efficiency}

\section{A.1 Trigger Frequency}

Tables A.1 and A.2 show the most frequently fired triggers for events which pass the cut0 pre-selection criteria.

\begin{tabular}{c|c}
\hline Trigger & Frequency (\%) \\
\hline \hline ML1_TMM5_VX & 14.3 \\
MM1_JT15_HA_TK10 & 11.2 \\
MM1_JT25 & 14 \\
MUJ1_JT25_LM3 & 13.3 \\
MUJ2_JT20_TK10 & 11.8 \\
MUW_W_L2M3_TRK10 & 15.1 \\
MU_2TRK3_L2M0 & 20.3 \\
MU_JT20_L2M0 & 18.1 \\
MU_JT25_L2M0 & 20.7 \\
\hline
\end{tabular}

Table A.1: Run 2a trigger frequency for the most frequently used triggers 


\begin{tabular}{c|c}
\hline Trigger & Frequency (\%) \\
\hline \hline M3_TMM3_VZDZ_NOLUM & 15.1 \\
M3_TMM3_VZ_NOLUM & 15.5 \\
M5_TLM3_2JBID_V & 15.3 \\
ML3_TMM4_VX & 34.9 \\
ML4_TMM5_VX & 26.3 \\
MUJ5_MM0_2TK3_VX & 38.6 \\
MUJ5_MM0_3T05L_V & 48.3 \\
MUJ5_MM0_3TK07_V & 48.3 \\
MUJ5_MM0_4TK05_V & 48.3 \\
MUJ5_MM0_BID_ & 26.8 \\
\hline
\end{tabular}

Table A.2: Run 2b trigger frequency for the most frequently used triggers

\section{A.2 File Locations}

The files which contain the trigger efficiencies are in the following locations:

- Run 2a jet trigger:

L1: jetid_eff/p17.09.03/TrigEff_Jet_CJT(1,5)_ncu_2D_deteta_pt_Binned.spc

- Run 2b jet trigger:

L1: jetid_eff/qcdjets/TrigEff_Jet_CSWJT(1,15,3.2)_ncu_2D_deteta_pt_Binned.spc

L2: jetid_eff/qcdjets/TrigEff_Jet_L2JET $(0,20)$ _50_2D_deteta_pt_Binned.spc

- Run 2a muon trigger:

L1: muid_eff/results_data/eff_Muon_11atxx_2D_phi_deteta_Binned.spc

L2: muid_eff/results_data/eff_Muon_12m0_2D_phi_deteta_Binned.spc

- Run 2b muon trigger:

L1: muid_eff/results_data/eff_Muon_l1atxx_2D_phi_deteta_Binned.spc

L2: muid_eff/results_data/eff_Muon_12m3_2D_phi_deteta_Binned.spc 

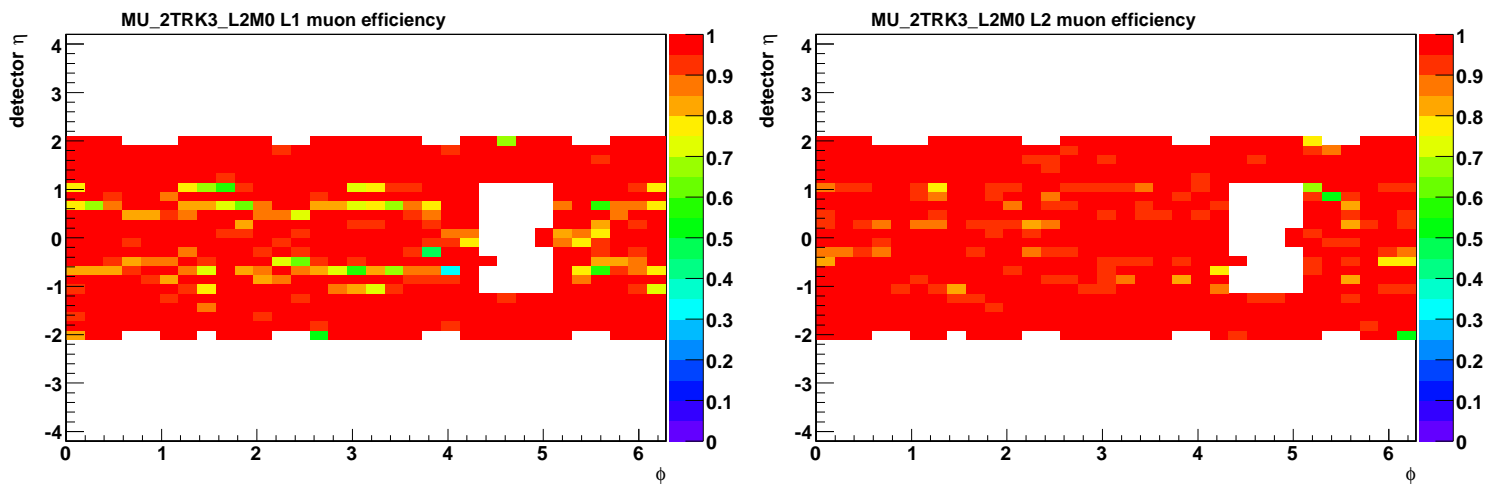

Figure A.1: The L1 and L2 efficiencies for the MU_2TRK3_L2M0 trigger with respect to an offline medium-quality, nseg $=3$ muon and a loose track.

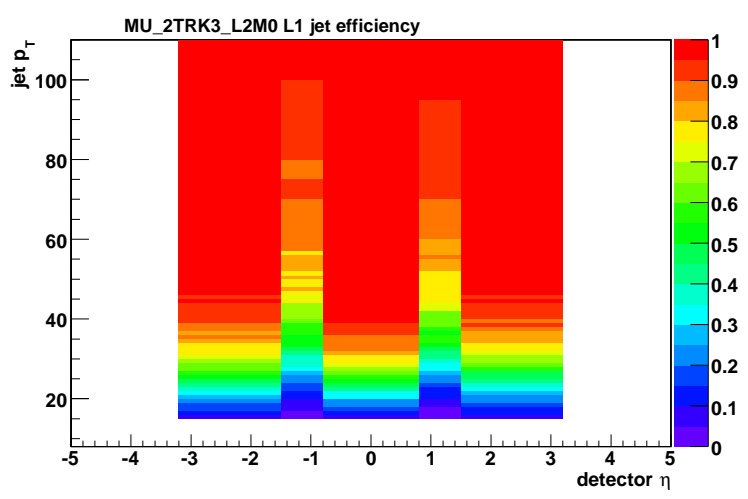

Figure A.2: The L1 efficiency of the MU_2TRK3_L2M0 trigger with respect to an offline good-jet.

\section{A.3 Trigger Efficiency Plots}

Figures A.1, A.2, A.3, and A.4 show the trigger efficiencies which are defined in the files in Appendix A.2. 

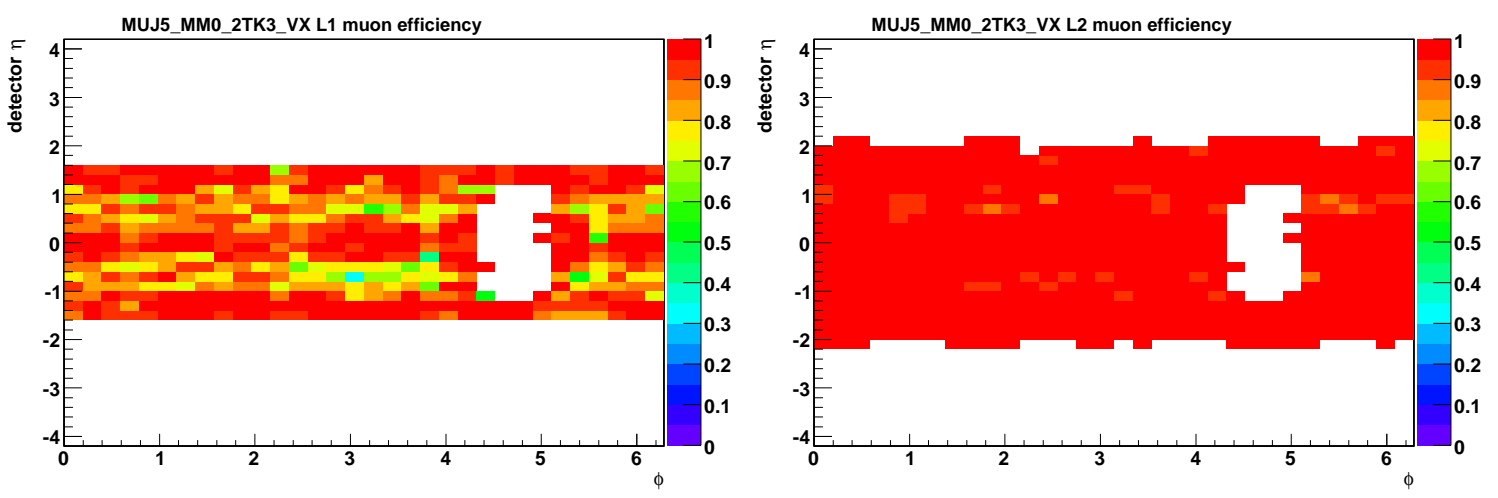

Figure A.3: The L1 and L2 efficiencies for the MUJ5_MM0_2TK3_VX trigger with respect to an offline medium-quality, nseg $=3$ muon and a loose track.
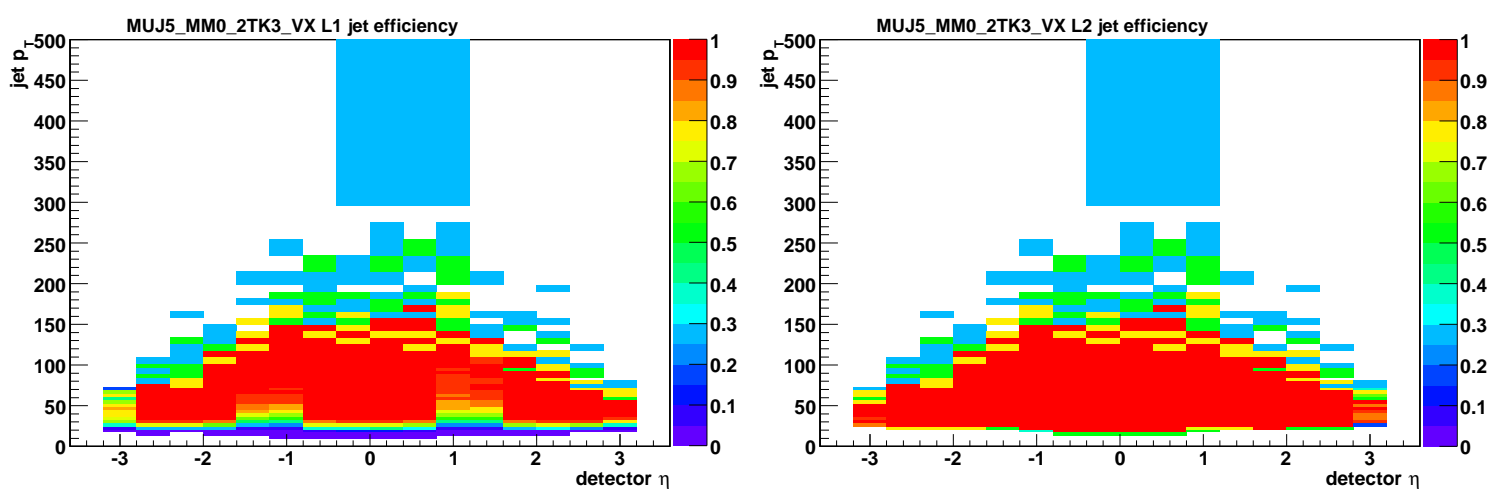

Figure A.4: The L1 and L2 efficiencies for the MUJ5_MM0_2TK3_VX trigger with respect to an offline good-jet. 


\section{Appendix B}

\section{Comparing p17 and p20 Data}

Figure B.1 compares data collected during run 2a to data collected during run $2 \mathrm{~b}$ for the variables which are important in this analysis. 

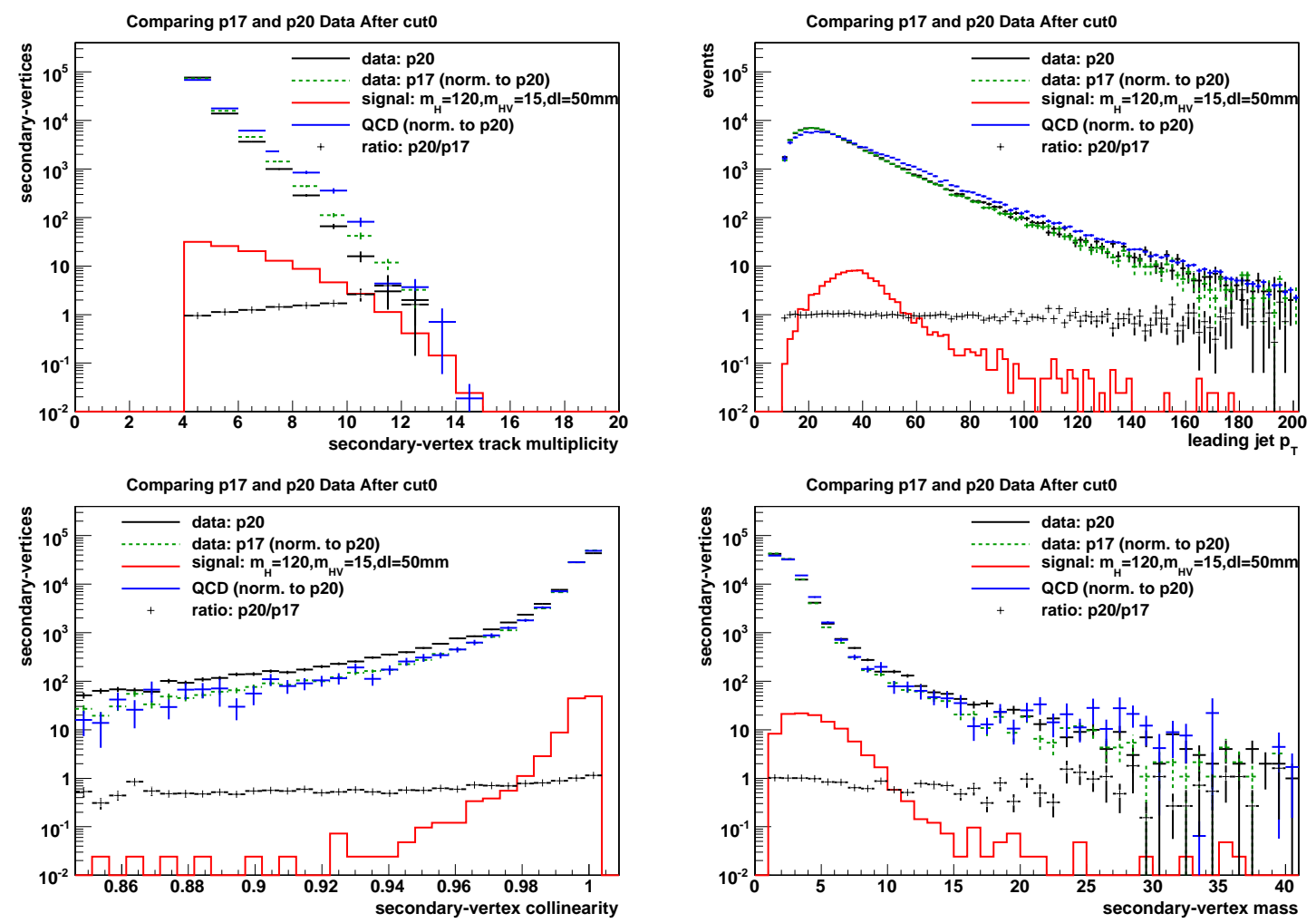

Figure B.1: Compare data collected during run 2a (p17) and run 2b (p20). 


\section{Appendix C}

\section{Secondary-vertex Density Cuts}

Figures C.1 and C.2 show plots before and after the material-density cuts for events which pass the cut0 pre-selection criteria. 

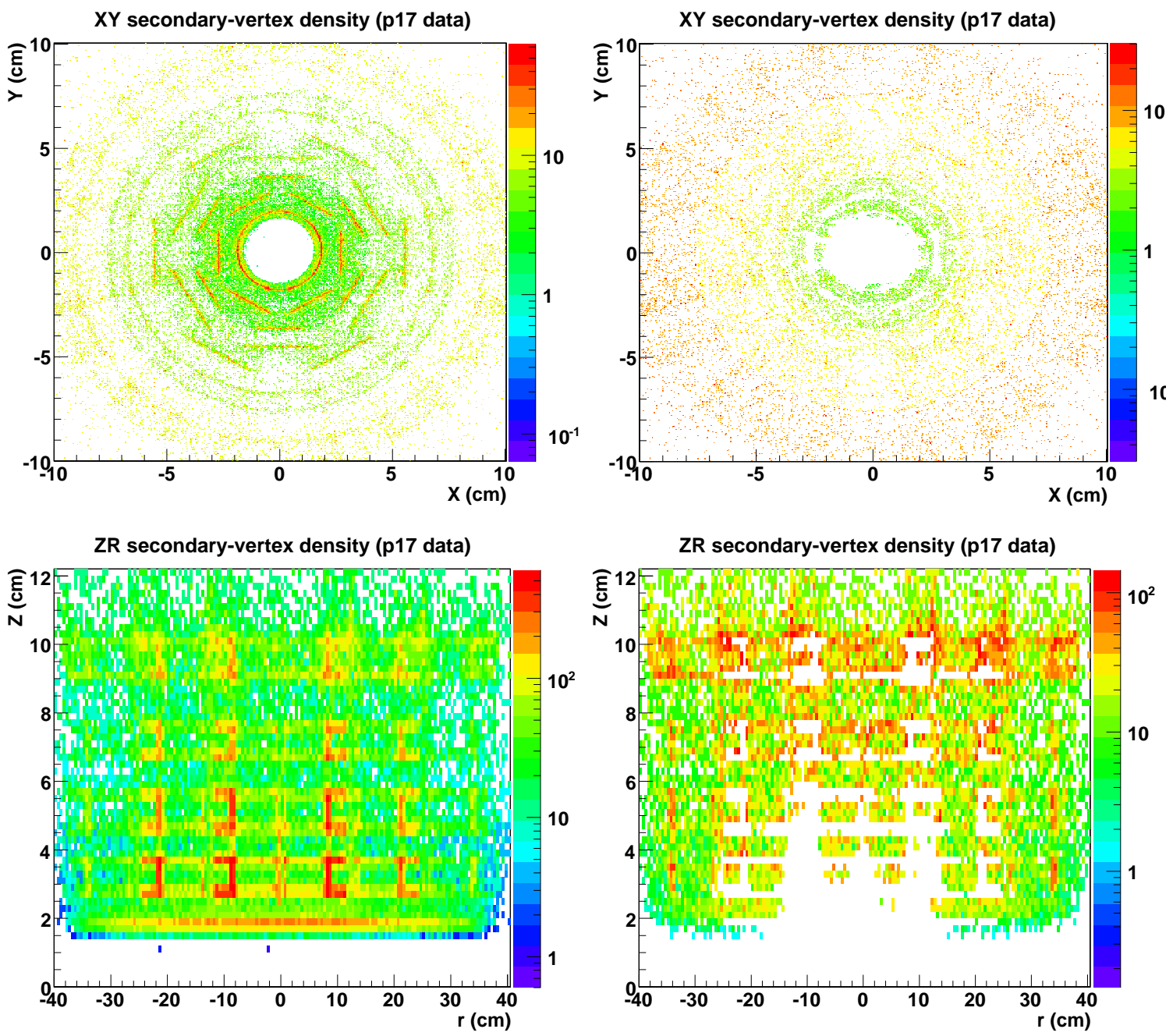

Figure C.1: Secondary-vertex density map of the $x y$ and $z r$-planes before and after cutting out areas of high SV density for the p17 data set. Secondary-vertices with track multiplicity $\geq 3$ are used to generate the map. The cut0 pre-selection criteria is required for all events in these plots. 

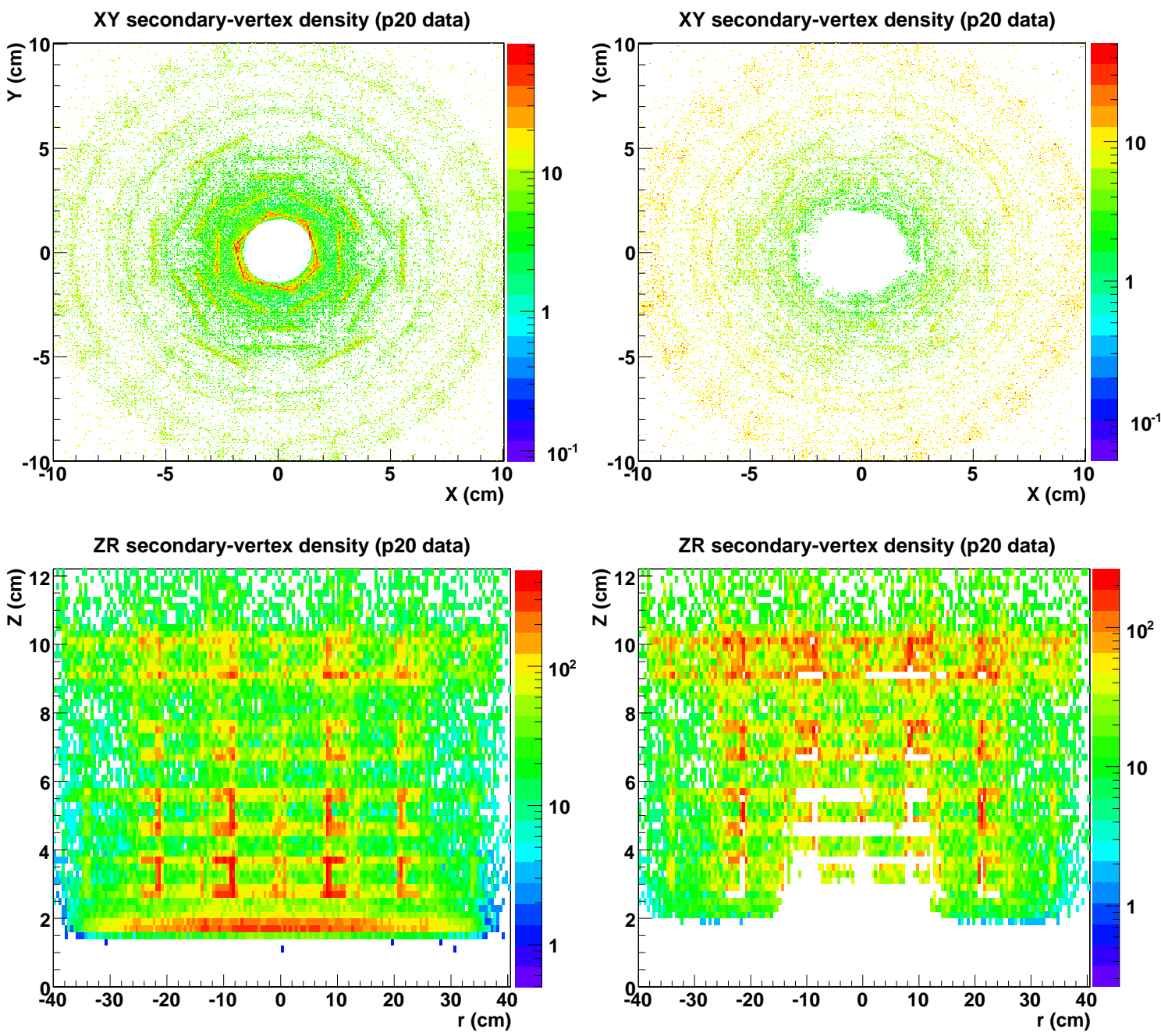

Figure C.2: Secondary-vertex density map of the $x y$ and $z r$-planes before and after cutting out areas of high SV density for the p20 data set. Secondary-vertices with track multiplicity $\geq 3$ are used to generate the map. The cut0 pre-selection criteria is required for all events in these plots. 


\section{Appendix D}

\section{Event Displays}

Figures D.2, D.3, D.4 and D.5 are event displays drawn using the D $\varnothing$ Visualization Environment (d0ve). 

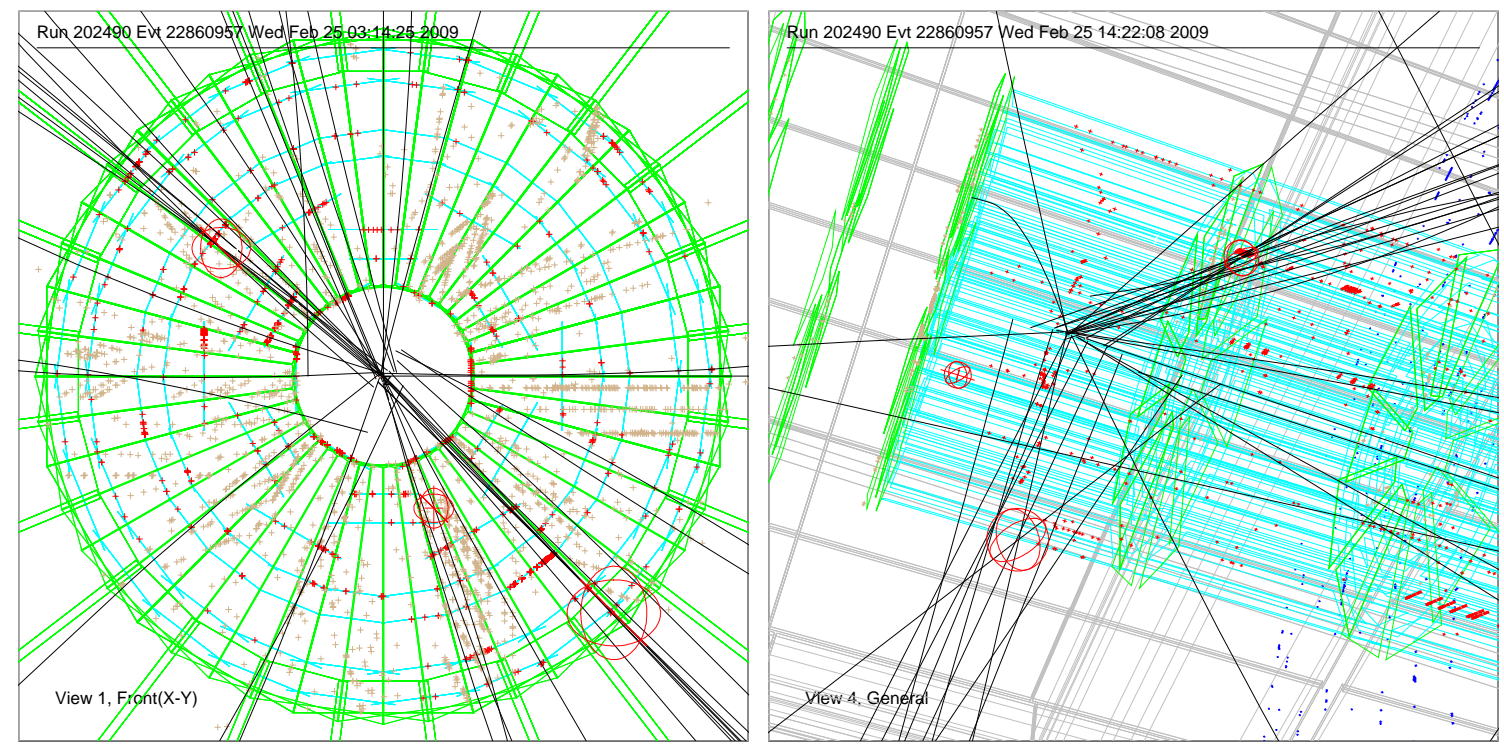

Figure D.1: Event displays in $x y$ and $z r$-planes for p17 data in which two secondaryvertices had collinearity $<0.9977$.

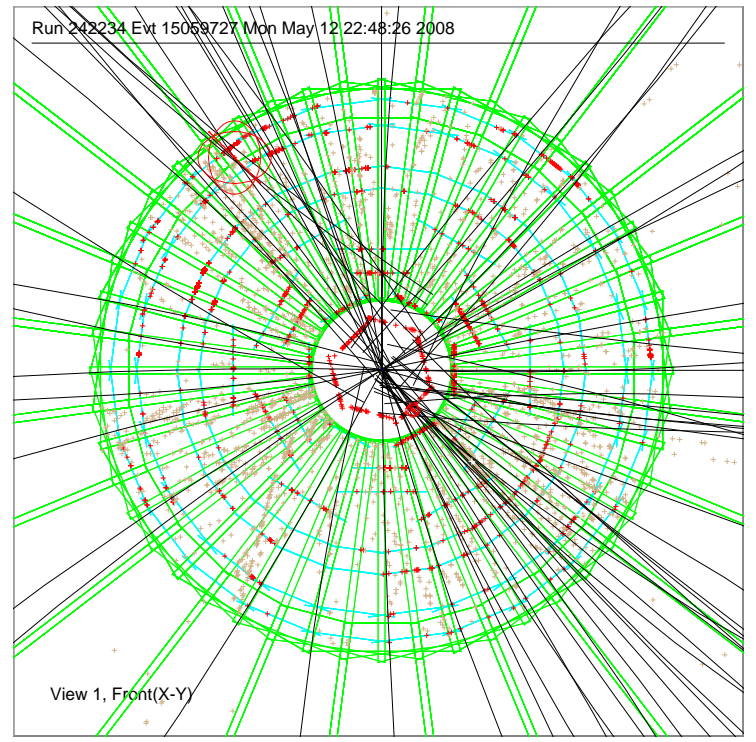

Run 242234 Evt 15059727 Mon May 12 22:48:26 2008

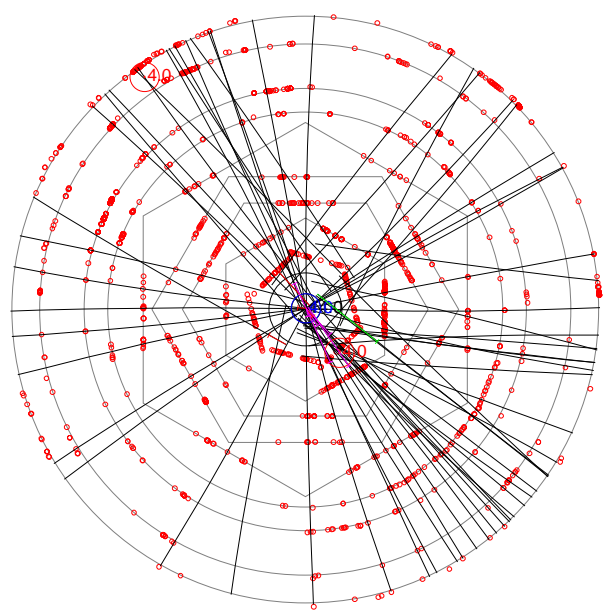

Figure D.2: Event displays in $x y$-plane for p20 data in which two secondary-vertices had collinearity $<0.9977$. 


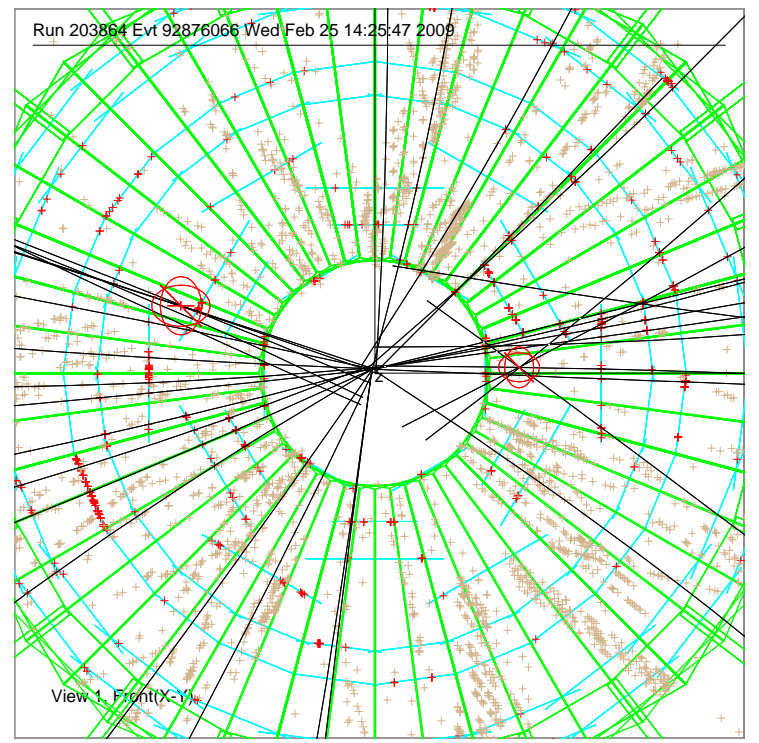

Run 203864 Evt 92876066 Wed Feb 25 03:18:12 2009

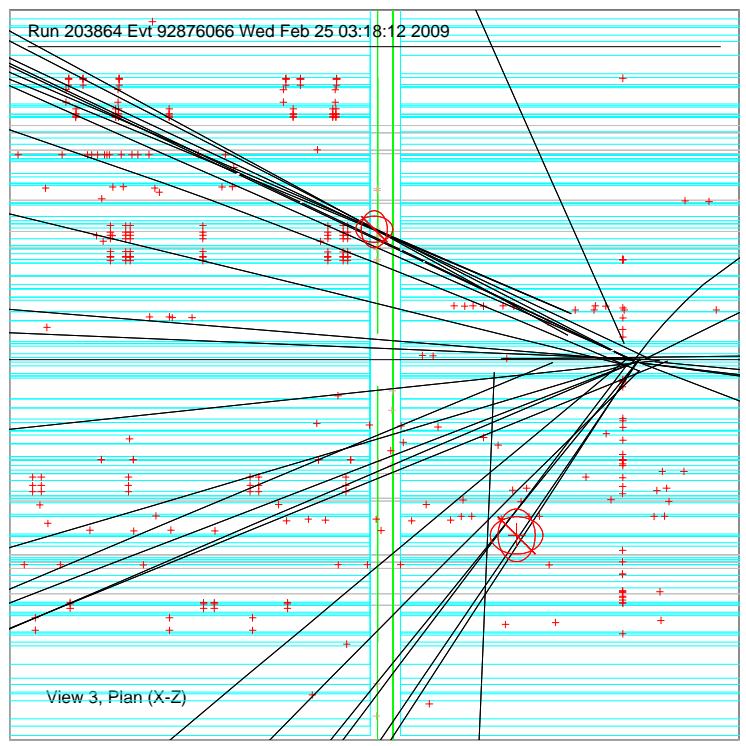

Figure D.3: Event displays in $x y$ and $z r$-planes for p17 data in which two secondaryvertices had mass $>2.5 \mathrm{GeV}$. 


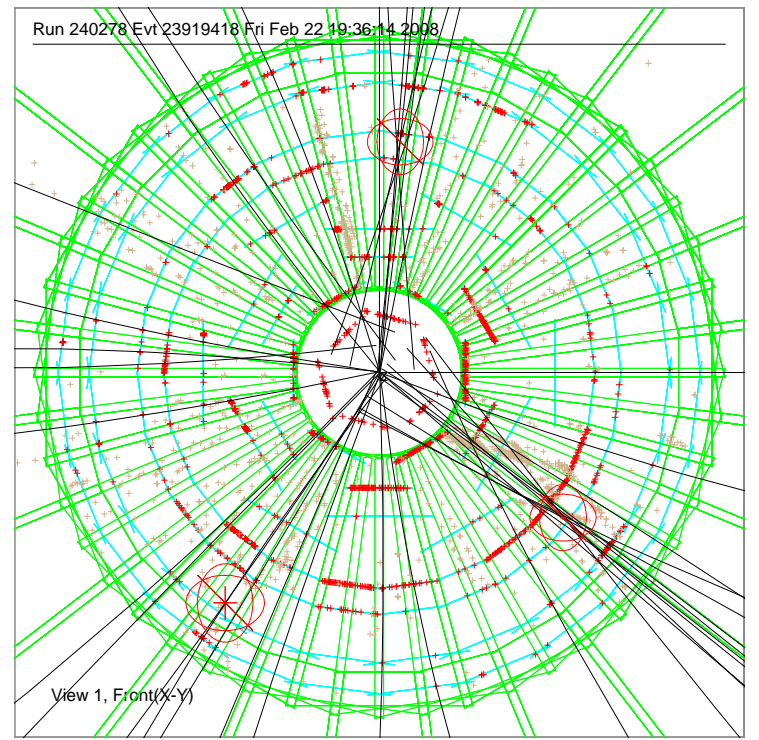

Run 240278 Evt 23919418 Fri Feb 22 19:36:14 2008
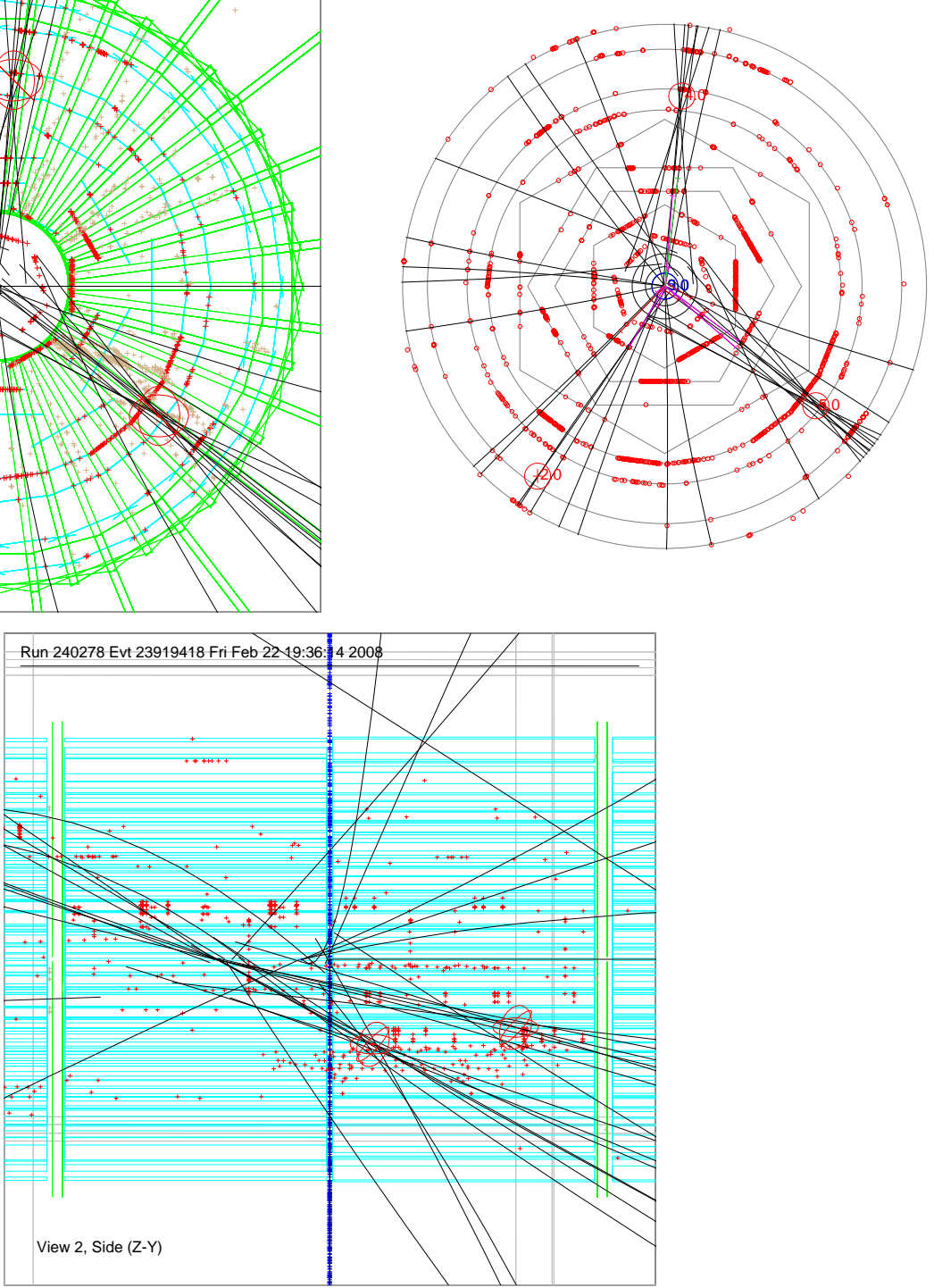

Figure D.4: Event displays in $x y$ and $z r$-planes for p20 data in which two secondaryvertices had mass $>2.5 \mathrm{GeV}$. 


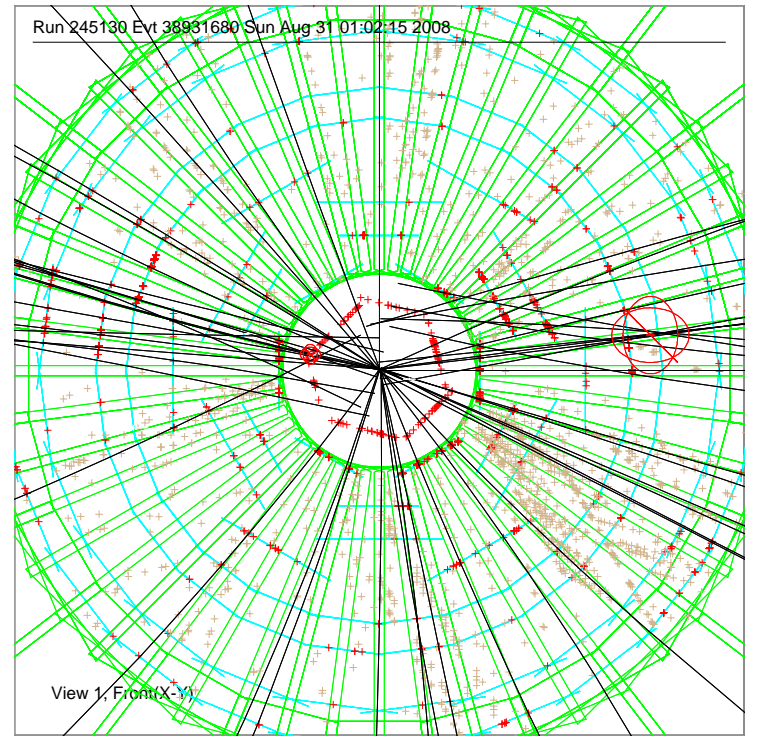

Run 245130 Evt 38931680 Sun Aug 31 01:02:15 2008

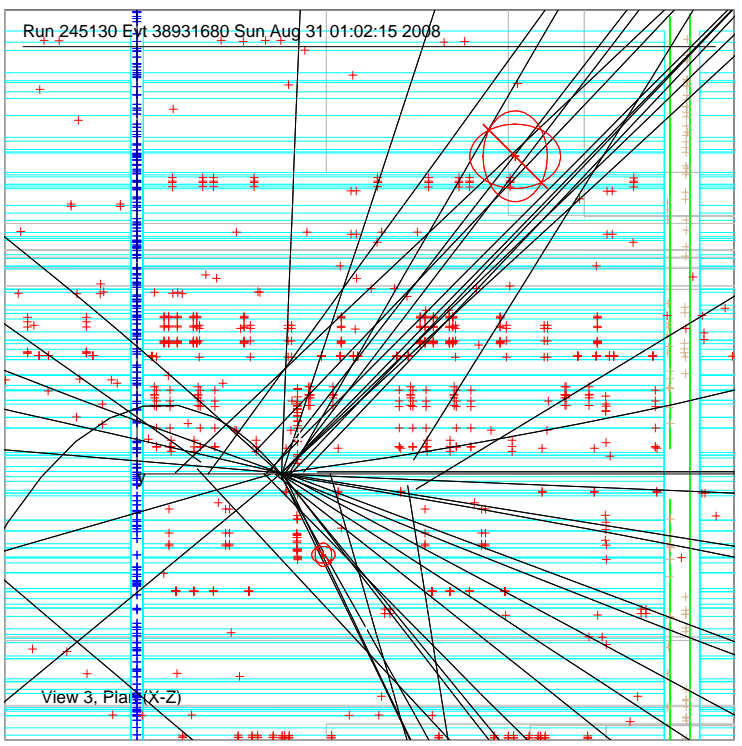

Figure D.5: Event displays in $x y$ and $z r$-planes for p20 data in which two secondaryvertices had mass $>2.5 \mathrm{GeV}$. 
Appendix E

Candidate Events 


\begin{tabular}{c||c|c||c|c}
\hline Dataset & p17 & & p20 & \\
SV property & SV 1 & SV 2 & SV 1 & SV 2 \\
\hline \hline collinearity & 0.99717 & 0.984148 & 0.979958 & 0.920021 \\
mass (GeV) & 1.51304 & 3.23739 & 1.42723 & 2.411 \\
multiplicity & 4 & 4 & 4 & 4 \\
xy density & 3.58265 & 6.40034 & 4.40059 & 14.9722 \\
zr density & 35.5992 & 27.2204 & 44.6877 & 45.5785 \\
$\chi^{2}$ & 12.0994 & 8.42303 & 15.1207 & 12.447 \\
attached muons & 0 & 1 & 0 & 0 \\
2d decay length & 6.28756 & 10.3739 & 9.66697 & 2.26722 \\
$\phi$ & 2.47728 & -0.799863 & 2.17996 & -0.888054 \\
$\eta$ & 0.983753 & 0.0233877 & -0.489197 & 0.0351337 \\
$\Delta R$ & 3.15573 & & 3.1125 & \\
\hline
\end{tabular}

Table E.1: Candidate events that pass the pre-selection criteria and maximum collinearity $<0.9977$. 


\begin{tabular}{c||c|c}
\hline Dataset & p17 & \\
SV property & SV 1 & SV 2 \\
\hline \hline collinearity & 0.999228 & 0.999373 \\
mass (GeV) & 3.13607 & 3.20391 \\
multiplicity & 4 & 4 \\
xy density & 9.27393 & 5.32185 \\
zr density & 24.6338 & 33.9927 \\
$\chi^{2}$ & 10.7155 & 1.7585 \\
attached muons & 0 & 0 \\
2d decay length $(\mathrm{cm})$ & 4.94491 & 3.60939 \\
$\phi$ & 2.82179 & 0.0120206 \\
$\eta$ & -0.615191 & -1.42852 \\
$\Delta R$ & 2.92512 & \\
\hline
\end{tabular}

\begin{tabular}{c||c|c||c|c}
\hline Dataset & p20 & & & \\
SV property & SV 1 & SV 2 & SV 1 & SV 2 \\
\hline \hline collinearity & 0.999725 & 0.999959 & 0.999079 & 0.998535 \\
mass (GeV) & 2.50203 & 3.54898 & 2.70078 & 2.72164 \\
multiplicity & 5 & 4 & 5 & 4 \\
xy density & 4.71381 & 4.69033 & 6.0517 & 3.48266 \\
zr density & 42.6499 & 54.7864 & 54.7864 & 25.081 \\
$\chi^{2}$ & 1.73224 & 8.99855 & 7.78696 & 1.20081 \\
attached muons & 0 & 0 & 0 & 0 \\
2d decay length $(\mathrm{cm})$ & 7.38794 & 7.3195 & 7.30774 & 12.144 \\
$\phi$ & -0.670351 & 1.48231 & 0.125487 & 3.05121 \\
$\eta$ & 0.856412 & 1.38243 & 0.677211 & 0.38319 \\
$\Delta R$ & 2.216 & \multicolumn{3}{|c}{} \\
\hline
\end{tabular}

Table E.2: Candidate events that pass the pre-selection criteria and minimum mass $>2.5 \mathrm{GeV}$. 